\title{
The $\mathrm{CN}$ isotopic ratios in comets ${ }^{\star, \star \star}$
}

\author{
J. Manfroid ${ }^{1, \star \star \star}$, E. Jehin ${ }^{1, \star \star \star}$, D. Hutsemékers ${ }^{1, \star \star \star}$, A. Cochran ${ }^{2}$, J.-M. Zucconi ${ }^{3, \dagger}$, C. Arpigny ${ }^{1}$, R. Schulz ${ }^{4}$, \\ J. A. Stüwe ${ }^{5}$, and I. Ilyin ${ }^{6}$
}

1 Institut d'Astrophysique et de Géophysique, Université de Liège, Allée du 6 août 17, 4000 Liège, Belgium e-mail: manfroid@astro.ulg.ac.be

2 Department of Astronomy and McDonald Observatory, University of Texas at Austin, C-1400, Austin, USA

3 Observatoire de Besançon, 25010 Besançon Cedex, France

${ }^{4}$ ESA/RSSD, ESTEC, PO Box 299, NL-2200 AG Noordwijk, The Netherlands

5 Leiden Observatory, 2300 RA Leiden, The Netherlands

6 AIP, An der Sternwarte 16, 14482 Potsdam, Germany

Received 17 February 2009 / Accepted 9 June 2009

ABSTRACT

\begin{abstract}
Our aim is to determine the isotopic ratios ${ }^{12} \mathrm{C} /{ }^{13} \mathrm{C}$ and ${ }^{14} \mathrm{~N} /{ }^{15} \mathrm{~N}$ in a variety of comets and link these measurements to the formation and evolution of the solar system. The ${ }^{12} \mathrm{C} /{ }^{13} \mathrm{C}$ and ${ }^{14} \mathrm{~N} /{ }^{15} \mathrm{~N}$ isotopic ratios are measured for the $\mathrm{CN}$ radical by means of high-resolution optical spectra of the $R$ branch of the $\mathrm{B}-\mathrm{X}(0,0)$ violet band. 23 comets from different dynamical classes have been observed, sometimes at various heliocentric and nucleocentric distances, in order to estimate possible variations of the isotopic ratios in parent molecules. The ${ }^{12} \mathrm{C} /{ }^{13} \mathrm{C}$ and ${ }^{14} \mathrm{~N} /{ }^{15} \mathrm{~N}$ isotopic ratios in $\mathrm{CN}$ are remarkably constant (average values of, respectively, $91.0 \pm 3.6$ and $147.8 \pm 5.7)$ within our measurement errors, for all comets whatever their origin or heliocentric distance. While the carbon isotopic ratio does agree with the terrestrial value (89), the nitrogen ratio is a factor of two lower than the terrestrial value (272), indicating a fractionation in the early solar system, or in the protosolar nebula, common to all the comets of our sample. This points towards a common origin of the comets independently of their birthplaces, and a relationship between HCN and CN.
\end{abstract}

Key words. comets: general - techniques: spectroscopic - line: identification - line: profiles - molecular processes

\section{Introduction}

Comets are believed to have condensed in the outer solar nebula and to contain relatively unaltered material from that period. The analysis of the composition of such material and particularly the determination of isotopic ratios should answer questions regarding the origin and the nature of the solar nebula.

This task is challenging. In-situ measurements in the coma with mass spectrometers are difficult, because heavy isotopes can be masked by hydrides, e.g., ${ }^{13} \mathrm{C}$ and ${ }^{12} \mathrm{CH},{ }^{15} \mathrm{~N}$ and ${ }^{14} \mathrm{NH}$. Values exist from in-situ measurements for the dust coma of comet $1 \mathrm{P} /$ Halley, for which the carbon isotopic ratio, ${ }^{12} \mathrm{C} /{ }^{13} \mathrm{C}$, varied by orders of magnitude from one grain to another (Solc et al. 1987; Jessberger \& Kissel 1991) - but no data were available for nitrogen.

Isotopic ratios can be determined remotely by highresolution spectroscopy of molecular bands. The emission lines of rare isotopes are weak and they have to be distinguished from emission lines of other isotopes, other species (e.g., $\mathrm{NH}_{2}$ in the case of the $\mathrm{C}_{2}$ Swan bands) and from the background. Likewise, blends are possible at sub-millimetre range: e.g., $\mathrm{SO}_{2}$ blends with the $\mathrm{H}^{13} \mathrm{CN}$ (4-3) line (Lis et al. 1997a). Hence, high

\footnotetext{
* Appendices and Table 8 are only available in electronic form at http://www . aanda.org

$\star \star$ Based on observations made with ESO Telescopes at the La Silla Paranal Observatory under programmes ID 268.C-5570, 270.C-5043, 073.C-0525, 274.C-5015 and 075.C-0355(A).

$\star \star \star$ J.M. is Research Director FNRS, E.J. is Research Associate FNRS and DH is Senior Research Associate FNRS.

$\dagger$ Deceased on 14 May 2009.
}

spectral resolution and high signal-to-noise ratios are needed. The accuracy of these determinations also depends on the models representing the emission by the various isotopologues, as well as other species appearing in the same domain.

Up to now, isotopic ratios have been published for $\mathrm{H}$, C, N, O and S (Jehin et al. 2006). The Rosetta mission to 67P/Churyumov-Gerasimenko will provide a detailed appraisal of the nature and isotopic composition of the material present at the surface of one comet. The present work addresses the $\mathrm{C}$ and $\mathrm{N}$ ground-based determinations that can now be routinely obtained for moderately bright comets.

\section{Historical background}

For carbon and nitrogen isotopes, the first observed molecules were $\mathrm{CN}$ and $\mathrm{C}_{2}$ in the optical and $\mathrm{HCN}$ in the sub-millimetre range. The first determinations of the ${ }^{12} \mathrm{C} /{ }^{13} \mathrm{C}$ ratio were obtained for bright comets from the ${ }^{12} \mathrm{C}^{13} \mathrm{C}(1,0)$ Swan bandhead at $4145 \AA$ (Stawikowski \& Greenstein 1964; Owen 1973). This feature was chosen because it is clearly separated from the corresponding ${ }^{12} \mathrm{C}^{12} \mathrm{C}$ bandhead. However, these measurements were affected by blending with $\mathrm{NH}_{2}$ emission. The cleaner CN B-X (0-0) band was used for the first time for comet 1P/Halley (Wyckoff \& Wehinger 1988; Wyckoff et al. 1989; Kleine et al. 1995), resulting in positive determinations of ${ }^{12} \mathrm{C} /{ }^{13} \mathrm{C}$. By 2000 , estimates of the ${ }^{12} \mathrm{C} /{ }^{13} \mathrm{C}$ ratio were available for ten comets. Although the same $\mathrm{CN}$ band lends itself to the determination of the nitrogen ratio, only lower limits were obtained. 
The nitrogen isotopic ratio ${ }^{14} \mathrm{~N} /{ }^{15} \mathrm{~N}$ was measured for the first time in comet C/1995 O1 (Hale-Bopp) in 1997 in the HCN as well as the $\mathrm{CN}$ band. The ${ }^{12} \mathrm{C} /{ }^{13} \mathrm{C}$ ratio was determined simultaneously. The ${ }^{12} \mathrm{C} /{ }^{13} \mathrm{C}$ and ${ }^{14} \mathrm{~N} /{ }^{15} \mathrm{~N}$ values derived from the $\mathrm{HCN}$ band at sub-millimetre range (respectively $111 \pm 12$ and $323 \pm 46$, Jewitt et al. 1997; $109 \pm 22$ and $330 \pm 98$, Ziurys et al. 1999), were consistent with the telluric values $(89,272$, Anders \& Grevesse 1989), although with large uncertainties. The ${ }^{12} \mathrm{C} /{ }^{13} \mathrm{C}$ values derived from $\mathrm{CN}\left({ }^{12} \mathrm{C} /{ }^{13} \mathrm{C}=100 \pm 35\right)$ were terrestrial, but the nitrogen isotopic ratio $\left({ }^{14} \mathrm{~N} /{ }^{15} \mathrm{~N}=140 \pm 35\right)$ was widely discordant with the terrestrial and $\mathrm{HCN}$ values (Arpigny et al. 2000, 2003).

Examination of published spectra of several other bright comets showed that ${ }^{12} \mathrm{C}^{15} \mathrm{~N}$ was probably also overabundant. The unexpected strength of these lines was a reason for discarding them as ${ }^{12} \mathrm{C}^{15} \mathrm{~N}$ (see, e.g., features labelled $\mathrm{k}, \mathrm{m}$ and $\mathrm{o}$ in the Halley spectrum displayed in Fig. 5 of Kleine et al. 1995, or lines $\mathrm{c}$ and $\mathrm{d}$ in the $\mathrm{C} / 1990 \mathrm{~K} 1$ spectrum in Fig. 8 of Wyckoff et al. 2000).

This discrepancy between $\mathrm{CN}$ and $\mathrm{HCN}$, a presumed parent, was eventually solved with the observation of $\mathrm{CN}$ and $\mathrm{HCN}$ in comet $17 \mathrm{P} /$ Holmes and the reanalysis of the Hale-Bopp submillimeter data (Bockelée-Morvan et al. 2008a) establishing that $\mathrm{HCN}$ has the same non-terrestrial nitrogen isotopic composition as $\mathrm{CN}$.

The seemingly conflicting results and the evidence for an anomalous value of ${ }^{14} \mathrm{~N} /{ }^{15} \mathrm{~N}$ had led us to initiate an observing campaign with the UVES high-resolution spectrograph (Dekker et al. 2000) mounted on the $8 \mathrm{~m}$ Kueyen telescope of the ESO VLT (Chile), in order to gather data on different comets presenting a variety of origins and physical conditions. The $\mathrm{CN} B^{2} \Sigma^{+}-$ $X^{2} \Sigma^{+}(0,0)$ violet band (near $3880 \AA$ ) was our main target.

Independently, in the Northern hemisphere, observations of many comets have been made since 1995 with the 2DCoudé high-resolution spectrograph of the $2.7 \mathrm{~m}$ telescope of the McDonald Observatory (Tull et al. 1995) and contributed a large proportion of our $\mathrm{CN}$ data.

\section{Our sample of comets and previous results}

So far, 23 comets have been observed (counting the fragments of $73 \mathrm{P} /$ Schwassmann-Wachmann 3 as one comet). They are listed in Table 2, with basic orbital characteristics including the Tisserand parameter $T_{\mathrm{J}}$ relative to Jupiter:

$T_{\mathrm{J}}=a_{\mathrm{J}} / a+2 \sqrt{\left(1-e^{2}\right) a / a_{\mathrm{J}}} \cos (i)$.

$a$ and $a_{\mathrm{J}}$ are the semi-major axes of the orbits of the comet and Jupiter, $e$ the eccentricity and $i$ the inclination of the comet's orbit. The Tisserand parameter is useful in comet taxonomy. Several classifications have been elaborated. We give in Table 2 the classifications according to Levison (1996) and Horner et al. (2003). The former proposes a major division at $T_{\mathrm{J}}=2$ between isotropic and ecliptic orbits. Further subdivision in our sample leads to Halley-type $\left(T_{\mathrm{J}}<2\right.$ and $\left.a<40 \mathrm{AU}\right)$, external $\left(T_{\mathrm{J}}<2\right.$ and $40<a<10000)$, new $\left(T_{\mathrm{J}}<2\right.$ and $\left.a>10000\right)$ and Jupiterfamily $\left(2<T_{\mathrm{J}}<3\right)$ comets. The comets of the first three groups probably come from the Oort cloud. The origin of the JF comets is less certain. In the Horner at al. scheme, for comets with perihelion closer than $4 \mathrm{AU}$, the classification reduces to four major types, Encke-type (E), short-period (SP), intermediate-period (I) and long-period (L), corresponding to aphelion divisions at 4 , 35 and $1000 \mathrm{AU}$. The objects are further differentiated according to the Tisserand parameter, with class I to IV defined by the boundaries 2.0, 2.5 and 2.8.
Our sample does not contain Levison's Encke-type objects (defined as $T_{\mathrm{J}}>3, a<a_{\mathrm{J}}$ ). Considering Horner's classification, $\mathrm{SP}_{\mathrm{II}}$ is missing and $\mathrm{SP}_{\mathrm{III}}$ is barely realized by the fragments of $73 \mathrm{P} / \mathrm{SW}-3$. The sample shows a majority of the long period objects (according to Horner's scheme) divided equally in external and new comets (Levison's scheme). There are two comets from the Halley family $\left(\mathrm{SP}_{\mathrm{I}}\right)$ and four from the Jupiter family (one $\mathrm{SP}_{\mathrm{III}}$ and three $\mathrm{SP}_{\mathrm{IV}}$ ), considering the two fragments of $73 \mathrm{P}$ as a single member.

We could not derive isotopic abundances for all 23 observed comets mainly because of the insufficient signal-to-noise ratio of the $\mathrm{CN}$ band, but 18 comets yielded positive measurements of both $\mathrm{C}$ and $\mathrm{N}$ ratios. Results have already been published partially for 10 of them. Our first target for UVES was comet C/2000 WM1 (LINEAR) which showed isotopic ratios compatible with those we had found in Hale-Bopp (Arpigny et al. 2000). This isotopic anomaly and the discrepancy with the $\mathrm{N}$ ratio determined from $\mathrm{HCN}$ in comet Hale-Bopp (Jewitt et al. 1997; Ziurys et al. 1999) led us to suggest the existence of parent(s) of CN other than HCN, with an even lower N isotopic ratio. Organic compounds like those found in interplanetary dust particles were proposed as possible candidates (Arpigny et al. 2003).

Our analysis of data gathered at McDonald observatory on 122P/de Vico and 153P/Ikeya-Zhang yielded isotopic ratios similar to Hale-Bopp and C/2000 WM1 (LINEAR) (Jehin et al. 2004).

Most observations were done at small heliocentric distances since this yields the brightest $m_{r}=m-5 \log (\Delta)$, i.e., the magnitude that would be seen from a distance of $1 \mathrm{AU}$ - a good indication of the flux entering the slit of the spectrometer. However, we gathered data on comets over a wider range of heliocentric distance in the hope of detecting variations of the isotopic ratios linked to different abundances of the parent species. It is likely that at large distances, the $\mathrm{CN}$ radical originates mainly from the photodissociation of HCN (see, e.g., Rauer et al. 2003). The isotopic ratios were determined in comets C/1995 O1 (HaleBopp), C/2001 Q4 (NEAT) and C/2003 K4 (LINEAR) at heliocentric distances of, respectively, 2.7, 3.7 and 2.6 AU (Manfroid et al. 2005) as well as close to 1 AU. No significant differences were found as a function of heliocentric distance, and the values were consistent with those obtained for other comets. The apparent discrepancy between the nitrogen isotopic ratio measurements in $\mathrm{CN}$ and $\mathrm{HCN}$ over such a range of irradiation conditions seemed to rule out $\mathrm{HCN}$ as a major parent of the cometary $\mathrm{CN}$ radicals.

The isotopic ratios were then determined for the first time in a Jupiter-family ( $\left.\mathrm{SP}_{\mathrm{IV}}\right)$ comet, 88P/Howell, and in the chemically peculiar Oort Cloud comet C/1999 S4 (LINEAR) (Hutsemékers et al. 2005). The carbon and nitrogen isotopic ratios agreed within the uncertainties with those already obtained.

The Deep Impact mission to comet 9P/Tempel 1 provided an extraordinary opportunity to observe from the ground the cometary material coming from relatively deep (a few meters) layers of the nucleus. The impact resulted in the release of subsurface material from the comet nucleus (A'Hearn et al. 2005), which formed a jet structure that expanded within the coma and was observable for several days after the impact. Observations of the activity and composition of the comet after the impact showed that the new material was compositionally different from that seen before impact and that the mass ratio of dust to gas in the ejecta was much larger than before (Meech et al. 2005). This suggested that the isotopic abundances could be different too. 
Observations of comet 9P/Tempel 1 were carried out before, during, and after the collision with the optical spectrometers UVES and HIRES mounted on the Kueyen telescope of the ESO VLT (Chile) and the Keck I telescope on Mauna Kea (Hawaii), respectively (Jehin et al. 2006). They show that the material released by the impact probably has the same carbon and nitrogen isotopic composition as the surface material, once again in line with the values derived so far.

Whether the material came from layers deep enough to be unaffected by space weathering remains unknown. If indeed the Deep Impact event led to the release of pristine material representative of the unaltered matter preserved in the interior of a comet nucleus since its formation, the measurement of the same isotopic composition in $\mathrm{CN}$ before and after impact would provide evidence for the ${ }^{12} \mathrm{C} /{ }^{13} \mathrm{C}$ and ${ }^{14} \mathrm{~N} /{ }^{15} \mathrm{~N}$ isotopic ratios being primordial in the parent molecule that produces $\mathrm{CN}$. However, infrared measurements obtained before and after impact showed an enhanced $\mathrm{C}_{2} \mathrm{H}_{6} / \mathrm{H}_{2} \mathrm{O}$ ratio, but an unchanged $\mathrm{HCN} / \mathrm{H}_{2} \mathrm{O}$ ratio relative to the quiescent (pre-impact) source (DiSanti \& Mumma 2008). It may therefore be that non-altered $\mathrm{CN}$-bearing material had not been reached by the impact. However, the processes that could affect the isotopic ratios are not the same as those affecting the relative amounts of volatiles near the surface. It is also possible that the nucleus is not differentiated in $\mathrm{HCN}$.

The 2007 outburst of the Jupiter Family comet 17P/Holmes allowed us to reconcile the $\mathrm{HCN}$ and $\mathrm{CN}$ data (Bockelée-Morvan et al. 2008a). Observation of $J=3-2$ rotational lines of $\mathrm{H}^{12} \mathrm{C}^{14} \mathrm{~N}, \mathrm{H}^{12} \mathrm{C}^{15} \mathrm{~N}$ and $\mathrm{H}^{13} \mathrm{C}^{14} \mathrm{~N}$ yielded ${ }^{14} \mathrm{~N} /{ }^{15} \mathrm{~N}=139 \pm 26$ while the measurements of the $\mathrm{CN}$ violet band gave ${ }^{14} \mathrm{~N} /{ }^{15} \mathrm{~N}=$ $165 \pm 40$. Moreover it appeared that the HCN ratios that had been published for Hale-Bopp were seriously overestimated. A reanalysis of the data gave $138<{ }^{14} \mathrm{~N} /{ }^{15} \mathrm{~N}<239$, and ${ }^{12} \mathrm{C} /{ }^{13} \mathrm{C}=94 \pm 8$. The isotopic ratios derived from $\mathrm{CN}$ and $\mathrm{HCN}$ are thus compatible within the error bars, and in line with those we measured in other comets.

In the following sections, we present a homogeneous compilation of the whole data set and revisit the previous results. Isotopic ratios of 8 comets are given for the first time. This completely new analysis uses, in particular, the latest fluorescence model including a more realistic handling of the collisional effects.

\section{Observations}

In order to study the carbon and nitrogen isotopes in cometary spectra, we chose to observe the $(0,0)$ violet band $(\mathrm{B}-\mathrm{X})$ of $\mathrm{CN}$. Figure 1 shows a typical (synthetic) ${ }^{12} \mathrm{C}^{14} \mathrm{~N}$ spectrum over the whole optical domain. The spectra of the heavier isotopologues have very similar distributions. The red bands are sparser than the blue ones, so the actual flux emitted in each band cannot be easily inferred from the figure. They are given in Table 1. Most of the optical emission occurs in the violet (0-0) band at $3880 \AA$ and in the red $\left(v^{\prime}-v^{\prime \prime}=1\right)$ bands between 9000 and $10000 \AA$. However, the red spectrum is more dispersed over many more lines.

To better appreciate which lines are the most useful, the brightest domains of the spectra of the three species $\left({ }^{12} \mathrm{C}^{14} \mathrm{~N}\right.$, ${ }^{13} \mathrm{C}^{14} \mathrm{~N}$ and $\left.{ }^{12} \mathrm{C}^{15} \mathrm{~N}\right)$ are shown in Figs. D.1-D.4.

Comparison with the atlas of comet de Vico (Cochran \& Cochran 2002) shows several of these isotopic lines among the unidentified features.

The B-X $(0,0)$ band appears to be the most interesting for our purposes. The lines are strong, and at that wavelength the solar spectrum is relatively weak. The red A-X bands are affected

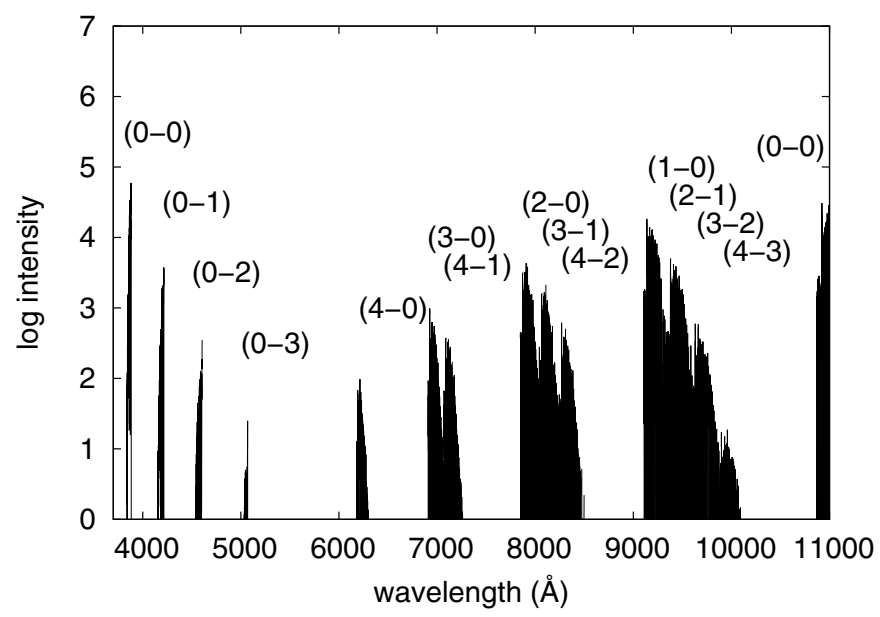

Fig. 1. Synthetic spectrum of ${ }^{12} \mathrm{C}^{14} \mathrm{~N}$ over the whole optical domain $(r=$ $1 \mathrm{AU}, \dot{r}=0)$. The $y$-axis shows the decimal logarithm of the relative intensities (arbitrary units). The blue bands below $5000 \AA$ A belong to the $\mathrm{B}-\mathrm{X}$ system, the red bands to the A-X system. The model is described in Sect. 6.

Table 1. Relative flux emitted in the various $\mathrm{CN}$ bands shown in Fig. 1.

\begin{tabular}{cr}
\hline \hline$v^{\prime}-v^{\prime \prime}$ & Flux \\
\hline 0 & 0.41717 \\
-1 & 0.02996 \\
-2 & 0.00192 \\
-3 & 0.00007 \\
4 & 0.00113 \\
3 & 0.01697 \\
2 & 0.11758 \\
1 & 0.41520 \\
\hline
\end{tabular}

by emission from other molecules (mainly $\mathrm{NH}_{2}$ and $\mathrm{C}_{2}-$ see, e.g., the de Vico atlas), a stronger solar background and complex absorption by atmospheric features $\left(\mathrm{H}_{2} \mathrm{O}, \mathrm{O}_{2}\right)$. The $\mathrm{B}-\mathrm{X}(0,1)$ band at $420 \mathrm{~nm}$ is also fainter and, hence, less useful for our purposes. Ideally, all these bands could be included in a global analysis to provide the best estimate of the isotopic ratios as well as the physical parameters in the coma but, in the present work, we limit ourselves to the bright (0-0) violet band.

High-resolution spectra are needed because the faint emission of the rare species are close to the strong lines coming from the abundant ones and because the best contrast to the underlying dust-scattered solar continuum must be obtained. Only the $R$ branch is of interest because the wavelength shifts in the $P$ branch are small compared to the typical spectral resolution and Doppler broadening of the lines.

The echelle spectrographs used in the present study were

- The UVES spectrograph at the Nasmyth focus of the $8 \mathrm{~m}$ Kueyen telescope (ESO/VLT, Paranal);

- SOFIN, the Cassegrain spectrograph at the $2.56 \mathrm{~m}$ NOT telescope (La Palma, Spain);

- the 2dCoudé echelle spectrograph at the $2.7 \mathrm{~m}$ Harlan F. Smith telescope of the McDonald Observatory (Texas);

- the HIRES spectrograph of the $10 \mathrm{~m}$ Keck I telescope (Hawaii). 
Table 2. Observed comets.

\begin{tabular}{|c|c|c|c|c|c|c|c|c|c|c|}
\hline \multirow[t]{2}{*}{ Comet } & \multirow{2}{*}{$\begin{array}{l}T_{\mathrm{P}} \\
\text { (yyyy-mm-dd) }\end{array}$} & \multirow[t]{2}{*}{$\bar{e}$} & \multirow{2}{*}{$\begin{array}{l}a \\
(\mathrm{AU})\end{array}$} & \multirow{2}{*}{$\begin{array}{l}q \\
(\mathrm{AU}) \\
\end{array}$} & \multirow{2}{*}{$\begin{array}{l}\text { Aph } \\
(\mathrm{AU})\end{array}$} & \multirow{2}{*}{$\begin{array}{l}i \\
\left({ }^{\circ}\right)\end{array}$} & \multirow{2}{*}{$\begin{array}{l}P \\
(\mathrm{yr})\end{array}$} & \multirow[t]{2}{*}{$\overline{\overline{T_{\mathrm{J}}}}$} & \multicolumn{2}{|c|}{ Type } \\
\hline & & & & & & & & & $\mathrm{L}$ & $\mathrm{H}$ \\
\hline $122 \mathrm{P} / \mathrm{de} \mathrm{Vico}^{a}$ & $1995-10-06$ & 0.96271 & 17.7 & 0.66 & 34.7 & 85 & 74.3 & 0.37 & $\mathrm{HF}$ & $\mathrm{SP}_{\mathrm{I}}$ \\
\hline C/1996 B2 (Hyakutake) & 1996-05-01 & 0.99990 & 2296 & 0.23 & 4591 & 125 & 110000 & -0.34 & EXT & $\mathrm{L}$ \\
\hline C/1995 O1 (Hale-Bopp) ${ }^{b, c}$ & 1997-04-01 & 0.99509 & 186.0 & 0.91 & 371.1 & 89 & 2537 & 0.04 & EXT & I \\
\hline 55P/Tempel-Tuttle & $1998-02-28$ & 0.90555 & 10.3 & 0.98 & 19.7 & 162 & 33.2 & -0.64 & $\mathrm{HF}$ & $\mathrm{SP}_{\mathrm{I}}$ \\
\hline C/1999 H1 (Lee) & 1999-07-11 & 0.99974 & 2773 & 0.71 & 5545 & 149 & 146000 & -0.90 & EXT & $\mathrm{L}$ \\
\hline C/1999 S4 (LINEAR) $)^{d}$ & $2000-07-26$ & 1.00010 & _- & 0.77 & _- & 149 & _- & - & NEW & $\mathrm{L}$ \\
\hline C/1999 T1 (McNaught-Hartley) & $2000-12-13$ & 0.99986 & 8502 & 1.17 & 17000 & 80 & 780000 & 0.23 & EXT & $\mathrm{L}$ \\
\hline C/2001 A2 (LINEAR) & $2001-05-25$ & 0.99969 & 2530 & 0.78 & 5060 & 36 & 127000 & 0.88 & EXT & $\mathrm{L}$ \\
\hline C/2000 WM1 (LINEAR) ${ }^{b}$ & $2002-01-23$ & 1.00025 & - & 0.56 & - & 73 & - & - & NEW & $\mathrm{L}$ \\
\hline 153P/Ikeya-Zhang ${ }^{a}$ & 2002-03-19 & 0.99010 & 51.2 & 0.51 & 101.9 & 28 & 366.5 & 0.88 & EXT & IP \\
\hline C/2002 X5 (Kudo-Fujikawa) & 2003-01-29 & 0.99984 & 1175 & 0.19 & 2350 & 94 & 40300 & -0.03 & EXT & $\mathrm{L}$ \\
\hline C/2002 V1 (NEAT) & 2003-02-18 & 0.99990 & 1010 & 0.10 & 2021 & 82 & 32100 & 0.06 & EXT & $\mathrm{L}$ \\
\hline C/2002 Y1 (Juels-Holvorcem) & $2003-04-13$ & 0.99715 & 250.6 & 0.71 & 500.5 & 104 & 3967 & -0.23 & EXT & I \\
\hline 88P/Howell ${ }^{d}$ & 2004-04-13 & 0.56124 & 3.1 & 1.37 & 4.9 & 4 & 5.5 & 2.95 & JF & $\mathrm{SP}_{\mathrm{IV}}$ \\
\hline C/2002 T7 (LINEAR) & 2004-04-23 & 1.00048 & - & 0.61 & - & 161 & - & - & NEW & $\mathrm{L}$ \\
\hline $\mathrm{C} / 2001 \mathrm{Q} 4(\mathrm{NEAT})^{c}$ & $2004-05-16$ & 1.00069 & _- & 0.96 & _- & 100 & _- & _- & NEW & $\mathrm{L}$ \\
\hline C/2003 K4 (LINEAR) ${ }^{c}$ & 2004-10-14 & 1.00030 & _- & 1.02 & _- & 134 & _- & - & NEW & $\mathrm{L}$ \\
\hline 9P/Tempel $1^{e}$ & 2005-07-05 & 0.51749 & 3.1 & 1.51 & 4.7 & 11 & 5.5 & 2.97 & JF & $\mathrm{SP}_{\mathrm{IV}}$ \\
\hline 73P-B/Schwassmann-Wachmann $3^{f}$ & 2006-06-08 & 0.69350 & 3.1 & 0.94 & 5.2 & 11 & 5.4 & 2.78 & JF & $\mathrm{SP}_{\mathrm{III}}$ \\
\hline 73P-C/Schwassmann-Wachmann $3^{f}$ & 2006-06-07 & 0.69338 & 3.1 & 0.94 & 5.2 & 11 & 5.4 & 2.78 & JF & $\mathrm{SP}_{\text {III }}$ \\
\hline C/2006 M4 (SWAN) & 2006-09-29 & 1.00018 & - & 0.78 & - & 112 & - & - & NEW & $\mathrm{L}$ \\
\hline 17P/Holmes ${ }^{g}$ & 2007-05-04 & 0.43242 & 3.6 & 2.05 & 5.18 & 19 & 6.9 & 2.86 & JF & $\mathrm{SP}_{\mathrm{IV}}$ \\
\hline 8P/Tuttle ${ }^{h}$ & 2008-01-27 & 0.8198 & 5.7 & 1.03 & 10.4 & 55 & 13.6 & 1.60 & $\mathrm{HF}$ & $\mathrm{SP}_{\mathrm{I}}$ \\
\hline C/2007 N3 (Lulin) & 2009-01-10 & 1.00004 & - & 1.21 & - & 178 & - & - & NEW & $\mathrm{L}$ \\
\hline
\end{tabular}

${ }^{a}$ Jehin et al. (2004) $-{ }^{b}$ Arpigny et al. (2000, 2003) $-{ }^{c}$ Manfroid et al. (2005) $-{ }^{d}$ Hutsemékers et al. (2005) $-{ }^{e}$ Jehin et al. (2006) $-{ }^{f}$ Jehin et al. (2008) $-{ }^{g}$ Bockelée-Morvan et al. (2008a) $-{ }^{h}$ Bockelée-Morvan et al. (2008b).

The elements are obtained from the JPL Solar System Dynamics website. $T_{\mathrm{P}}$ is the epoch of the perihelion, $e$ the eccentricity, $q$ the perihelion distance in AU, Aph the aphelion distance, $i$ the inclination on the ecliptic, $P$ the period, $T_{\mathrm{J}}$ the Tisserand parameter relative to Jupiter, the last columns give the classification according to Levison (1996) and Horner et al. (2003). The subdivision I-IV based on $T_{\mathrm{J}}$ is given for the SP comets only, all others being of the sub-type I.

The circumstances of the various observing runs are summarized in Table 3, while the data relative to individual spectra are given in Table 8.

\section{Data reduction}

We used the echelle package of the IRAF software (NOAO) to calibrate the spectra and to extract the relevant order(s) of the $\mathrm{CN}$ violet band. In the case of the VLT spectra, the UVES configuration is such that the $\mathrm{CN}$ band appears in two orders. The SOFIN and 2DCoudé spectra do not suffer from the same problem: all of the band is in a single order. The UVES wavelength calibration was done on the $\mathrm{CN}$ lines themselves in order to avoid possible shifts between the science spectra and calibration lamp spectra. Indeed, the UVES calibration errors measured between the $\mathrm{CN}$ and lamp solutions show an rms scatter of $0.66 \mathrm{~km} \mathrm{~s}^{-1}$.

The echelle orders were combined using an optimal scheme taking into account the low levels expected for the isotopic lines - i.e., a regime where the signal-to-noise ratio is directly proportional to the signal - and simultaneously correcting the echelle ripple (blaze function). The wavelength domain is very small, $\sim 10 \AA$, so that a normalization is done in such a way that flat-field spectra extracted in the same conditions are constant. This procedure yields the "flat-field normalized" spectrum $o_{\mathrm{ff}}$ and the $\mathrm{S} / \mathrm{N}$ ratio expected for a low-level constant signal, $f(\lambda)$. The $\mathrm{S} / \mathrm{N}$ ratios of faint lines of intensity $I$ can be directly compared using

$\frac{\mathrm{SN}_{i}}{\mathrm{SN}_{j}}=\frac{I_{i} f\left(\lambda_{i}\right)}{I_{j} f\left(\lambda_{j}\right)}$

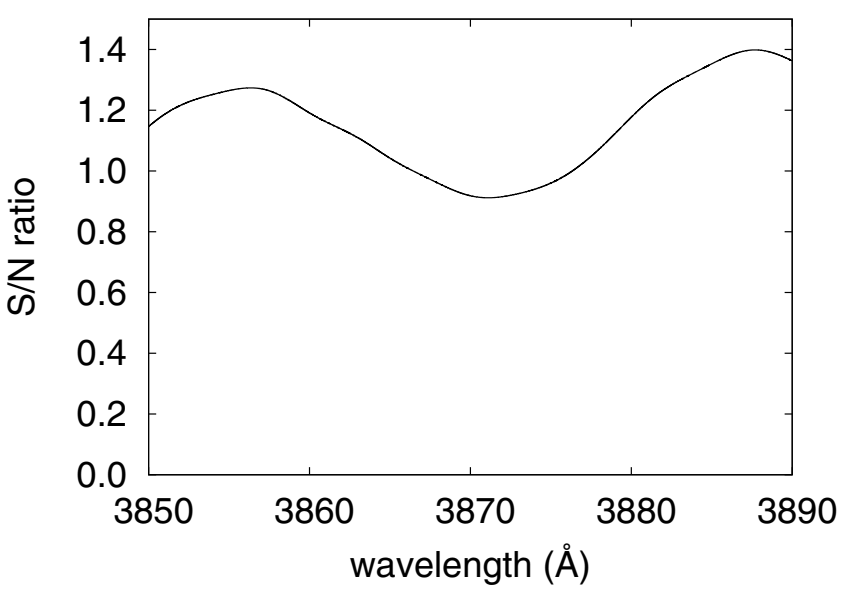

Fig. 2. Variations of of the signal-to-noise ratio $f(\lambda)$ across the UVES $\mathrm{CN}$ spectra for low-level signals.

Alternatively, a "S/N-normalized" spectrum $o_{\mathrm{sn}}(\lambda)$ can be produced, such that the intensity of faint lines is directly proportional to their $\mathrm{S} / \mathrm{N}$ ratio

$o_{\mathrm{sn}}(\lambda)=o_{\mathrm{ff}}(\lambda) f(\lambda)$

A typical $f(\lambda)$ is presented in Fig. 2. The minimum falls on the $\mathrm{CN}$ band, in the overlapping region between two echelle orders, and corresponds approximately to a $2^{-1 / 2}$ loss. Unfortunately, changing the relevant UVES settings so as to bring the $\mathrm{CN} R$ band in the middle of one order was not possible. 
Table 3. Observational circumstances.

\begin{tabular}{|c|c|c|c|c|c|c|c|c|c|c|c|}
\hline Run & $\begin{array}{l}\text { JD } \\
2400000+ \\
\end{array}$ & Comet & $\begin{array}{c}r \\
(\mathrm{AU}) \\
\end{array}$ & $\begin{array}{c}\dot{r} \\
(\mathrm{~km} / \mathrm{s}) \\
\end{array}$ & $\begin{array}{c}\Delta \\
(\mathrm{AU})\end{array}$ & $\begin{array}{c}\Delta \\
(\mathrm{km} / \mathrm{s})\end{array}$ & Spectro & 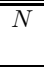 & $\begin{array}{l}\operatorname{Exp} \\
(\mathrm{ks})\end{array}$ & $\overline{\bar{R}}$ & $\begin{array}{c}\text { Slit } \\
\left({ }^{\prime \prime} \times{ }^{\prime \prime}\right)\end{array}$ \\
\hline 1 & 49993:49994 & $122 \mathrm{P} / \mathrm{de}$ Vico & 0.66 & $-2.89:-1.75$ & $0.99: 1$ & $-14.3:-12.87$ & 2DCoudé & 4 & 3.9 & 60000 & $1.2 \times 8.2$ \\
\hline 2 & 50151 & C/1996 B2 (Hyakutake) & 1.35 & $-32.94:-32.93$ & 0.53 & $-57.03:-56.96$ & 2DCoudé & 2 & 3.6 & 60000 & $1.2 \times 8.2$ \\
\hline 3 & 50175 & C/1996 B2 (Hyakutake) & 0.87 & $-38.69:-38.68$ & 0.27 & 53.08:53.14 & 2DCoudé & 3 & 5.4 & 200000 & $1.2 \times 8.2$ \\
\hline 4 & $50367: 50374$ & C/1995 O1 (Hale-Bopp) & $2.68: 2.77$ & $-20.78:-20.62$ & $3.02: 3.04$ & $3.81: 5.94$ & 2DCoudé & 17 & 30.6 & 60000 & $1.2 \times 8.2$ \\
\hline 5 & 50544 & C/1995 O1 (Hale-Bopp) & 0.91 & $2.98: 3$ & 1.39 & $18.52: 18.55$ & 2DCoudé & 3 & 2.8 & 200000 & $1.2 \times 8.2$ \\
\hline 6 & $50545: 50551$ & C/1995 O1 (Hale-Bopp) & $0.92: 0.94$ & $4.04: 7.47$ & $1.41: 1.49$ & $20.23: 24.83$ & SOFIN & 23 & 32.5 & 70000 & $0.4 \times 3.8$ \\
\hline 7 & 50830:50831 & 55P/Tempel-Tuttle & $1.18: 1.19$ & $-15.5:-15.28$ & 0.35 & $-2.37: 4.96$ & 2DCoudé & 2 & 3.6 & 60000 & $1.2 \times 8.2$ \\
\hline 8 & 51444 & C/1999 H1 (Lee) & 1.54 & 24.94 & 0.85 & -13.13 & 2DCoudé & 1 & 1.8 & 60000 & $1.2 \times 8.2$ \\
\hline 9 & $51720: 51721$ & C/1999 S4 (LINEAR) & $0.96: 0.97$ & $-19.6:-19.3$ & $1.15: 1.19$ & $-62.3:-61.9$ & 2DCoudé & 2 & 3.6 & 60000 & $1.2 \times 8.2$ \\
\hline 10 & $51731: 51742$ & C/1999 S4 (LINEAR) & $0.78: 0.86$ & $-14.9:-7.4$ & 0.44:0.79 & $-63.2:-40$ & 2DCoudé & 14 & 22.8 & 60000 & $1.2 \times 8.2$ \\
\hline 11 & 51932:51935 & C/1999 T1 (McNaught-Hartley) & $1.34: 1.36$ & 12.81:13.44 & $1.29: 1.3$ & $-6.73:-4.87$ & 2DCoudé & 11 & 16.4 & 60000 & $1.2 \times 8.2$ \\
\hline 12 & 52114 & C/2001 A2-A (LINEAR) & 1.36 & 23.59 & 0.42 & 21.92 & 2DCoudé & 1 & 1.8 & 60000 & $1.2 \times 8.2$ \\
\hline 13 & $52340: 52341$ & C/2000 WM1 (LINEAR) & 1.08:1.1 & $28.25: 28.26$ & 1.23 & $0.12: 0.28$ & UVES & 4 & 6.2 & 80000 & $0.45 \times 8$ \\
\hline 14 & $52355: 52356$ & C/2000 WM1 (LINEAR) & $1.32: 1.34$ & $27.83: 27.88$ & 1.23 & $-0.25:-0.12$ & UVES & 4 & 6.3 & 80000 & $0.45 \times 8$ \\
\hline 15 & 52386 & 153P/Ikeya-Zhang & 0.92 & 29.22 & 0.41 & $-6.44:-6.39$ & 2DCoudé & 3 & 3.6 & 60000 & $1.2 \times 8.2$ \\
\hline 16 & $52647: 52649$ & C/2002 V1 (NEAT) & $1.17: 1.22$ & $-37.11:-36.51$ & $0.83: 0.84$ & $7.86: 8.32$ & UVES & 4 & 8.2 & 80000 & $0.45 \times 8$ \\
\hline 17 & $52689: 52690$ & C/2002 X5 (Kudo-Fujikawa) & 0.69:0.72 & $42.6: 43.03$ & 0.86 & $-5.09:-2.5$ & UVES & 3 & 5.1 & 80000 & $0.45 \times 8: 12$ \\
\hline 20 & $52788: 52789$ & C/2002 Y1 (Juels-Holvorcem) & $1.14: 1.15$ & $24.09: 24.18$ & 1.55 & $-7.23:-7.18$ & UVES & 4 & 7.2 & 80000 & $0.4 \times 8$ \\
\hline 21 & $52883: 52889$ & C/2001 Q4 (NEAT) & $3.66: 3.73$ & $-18.91:-18.8$ & $3.36: 3.45$ & $-25.43:-23.55$ & UVES & 4 & 23.4 & 80000 & $0.45 \times 8$ \\
\hline 22 & $53113: 53117$ & $88 \mathrm{P} /$ Howell & 1.37 & $0.89: 1.5$ & $1.66: 1.67$ & $-4.5:-4.09$ & UVES & 4 & 14.4 & 80000 & $0.44 \times 8$ \\
\hline 23 & $53127: 53129$ & $88 \mathrm{P} /$ Howell & $1.38: 1.39$ & $3: 3.27$ & 1.64 & $-3.3:-3.15$ & UVES & 3 & 10.8 & 80000 & $0.44 \times 8$ \\
\hline 24 & $53130: 53132$ & C/2001 Q4 (NEAT) & 0.97 & $-5.43:-4.84$ & 0.32 & $-4.12: 2.23$ & UVES & 10 & 20.6 & 80000 & $0.44 \times 8$ \\
\hline 25 & $53131: 53132$ & C/2002 T7 (LINEAR) & $0.68: 0.69$ & $15.8: 16.7$ & $0.56: 0.6$ & $-65.59:-64.8$ & UVES & 4 & 3.7 & 80000 & $0.44 \times 8$ \\
\hline 26 & 53132 & C/2003 K4 (LINEAR) & 2.59 & -20.35 & 2.35 & -43 & UVES & 1 & 4.3 & 60000 & $0.6 \times 8$ \\
\hline 27 & $53142: 53149$ & 88P/Howell & $1.42: 1.44$ & $5.04: 5.9$ & $1.61: 1.62$ & $-2.7:-2.6$ & UVES & 4 & 14.5 & 80000 & $0.44 \times 8$ \\
\hline 28 & $53150: 53153$ & C/2002 T7 (LINEAR) & $0.92: 0.97$ & $25.4: 25.9$ & $0.38: 0.48$ & $51.94: 59.3$ & UVES & 6 & 15.6 & 80000 & $0.40: 0.44 \times 8$ \\
\hline 29 & $53166: 53169$ & C/2002 T7 (LINEAR) & $1.17: 1.21$ & $26.84: 26.87$ & $0.95: 1.06$ & 64.03:64.45 & UVES & 5 & 15.7 & 80000 & $0.44 \times 8$ \\
\hline 30 & 53328:53329 & C/2003 K4 (LINEAR) & 1.19:1.2 & $14.55: 14.81$ & $1.51: 1.52$ & $-28.26:-28.2$ & UVES & 2 & 3 & 80000 & $0.44 \times 10$ \\
\hline 31 & 53520 & $9 \mathrm{P} /$ Tempel 1 & 1.55 & -4 & 0.75 & $5.09: 5.2$ & HIRES & 3 & 3.6 & 47000 & $0.8 \times 7$ \\
\hline 32 & $53523:$ & 9P/Tempel 1 & $1.53: 1.54$ & $-3.7:-3.1$ & $0.76: 0.78$ & $5.3: 6.3$ & UVES & 6 & 32.4 & 80000 & $0.44 \times 10$ \\
\hline 33 & $53553: 5$ & 9P/Tempel 1 & 1.51 & $-0.3: 0.8$ & $0.88: 0.93$ & $8.9: 10.3$ & UVES & 20 & 147.6 & 80000 & $0.44 \times 10$ \\
\hline 34 & 53555 & 9P/Tempel 1 & 1.51 & $-9.1:-0.1$ & 0.89 & $9.1: 9.4$ & HIRES & 14 & 13.6 & 47000 & $0.8 \times 7$ \\
\hline 35 & $53857: 53860$ & 73P-B/Schwassmann-Wachmann 3 & $1.05: 1.07$ & $-11.76:-11.06$ & 0.09:0.11 & $-11.3:-9.19$ & 2DCoudé & 2 & 2.1 & 60000 & $1.2 \times 8.2$ \\
\hline 36 & $53858: 53860$ & 73P-C/Schwassmann-Wachmann 3 & $1.05: 1.07$ & $-11.77:-11.33$ & $0.1: 0.11$ & $-12.1:-11.2$ & 2DCoudé & 3 & 5.4 & 60000 & $1.2 \times 8.2$ \\
\hline 37 & $53882: 53883$ & 73P-B/Schwassmann-Wachmann 3 & 0.95 & $-4.17:-3.79$ & 0.15 & $12.31: 12.53$ & UVES & 3 & 10.8 & 80000 & $0.6 \times 10$ \\
\hline 40 & 54039 & C/2006 M4 (SWAN) & 0.99 & $19.48: 19.5$ & 1.02 & $13.2: 13.3$ & 2DCoudé & 2 & 3.6 & 60000 & $1.2 \times 8.2$ \\
\hline 41 & 54398:54402 & 17P/Holmes & $2.44: 2.46$ & 5 & 1.63 & $-3.8:-2.6$ & 2DCoudé & 5 & 2.7 & 60000 & $1.2 \times 8.2$ \\
\hline 42 & 54402 & 17P/Holmes & 2.46 & 6.65 & 1.63 & -2.4 & HIRES & 1 & 0 & 47000 & $0.8 \times 7$ \\
\hline 43 & 54403:54404 & $17 \mathrm{P} /$ Holmes & 2.46 & 5 & 1.62 & $-2.2:-1.9$ & 2DCoudé & 2 & 2.4 & 60000 & $1.2 \times 8.2$ \\
\hline 44 & $54422: 54423$ & 17P/Holmes & 2.54 & 6.94 & 1.64 & $5: 5.2$ & 2DCoudé & 7 & 12.6 & 60000 & $1.2 \times 8.2$ \\
\hline 45 & $54481: 54500$ & $8 \mathrm{P} /$ Tuttle & $1.03: 1.04$ & $-4.34: 3.13$ & $0.35: 0.62$ & $21.64: 24.79$ & UVES & 6 & 24 & 80000 & $0.44 \times 10$ \\
\hline 46 & $54876: 54877$ & C/2007 N3 (Lulin) & 1.33 & $10.72: 11$ & 0.53 & $-43:-39.7$ & 2DCoude & 17 & 30.6 & 60000 & $1.2 \times 8.2$ \\
\hline
\end{tabular}

For convenience the data are grouped in observing runs. $r$ is the heliocentric distance in astronomical units (AU), $\dot{r}$ the heliocentric radial velocity, $\Delta$ is the geocentric distance, $\dot{\Delta}$ the topocentric radial velocity, "Spectro" the spectrograph used, $N$ is the number of individual spectra, "Exp" the total exposure time in thousands of seconds, $R=\Delta \lambda / \lambda$ the resolving power. The nominal size of the entrance slit of the spectrograph is given in arcsec. Specific values relative to individual spectra can be found in Table 8 .

The dust-reflected sunlight and, in some cases, the twilight contamination and the lunar sky background underlying the cometary emissions, were removed from the $o_{\mathrm{ff}}$ by fitting a solar reference spectrum to each of these components. Moon or twilight sky spectra were often used for this purpose as they directly provided correct line profiles, but other good high-resolution solar spectra can be used, such as the UVES reference solar spectrum produced by ESO or the atlas by Delbouille et al. (1973), available online at the BASS2000 site (http://bass2000. obspm. fr/solar_spect.php).

Appropriate Doppler shifts had to be applied since, at the resolution of the echelle spectra, the observer, the Moon, the Sun and the comet have non-negligible relative velocities.

- The Moon spectrum and the lunar sky background show a radial velocity equal to the sum of the heliocentric and topocentric velocities of the Moon;
- the dust-reflected sunlight shows a radial velocity equal to the sum of the heliocentric and topocentric velocities of the comet;

- the twilight component is shifted by the topocentric velocity of the Sun;

- the cometary emission is shifted by the topocentric velocity of the comet.

The relevant kinematic data were retrieved from the JPL Horizon ephemeris generator. Because of the Earth rotation, the topocentric velocities show the most rapid variations. Generally, the use of average values is sufficient even when correcting the longest exposures. However, the variations of the twilight are so fast that the Doppler shift corresponds to a slightly different time than the other components (earlier in the evening, later in the morning). The spectra of the Moon rising or setting during long exposures sometimes needs similar adjustments. 
The transfer functions (line profiles) of those components are not identical. The profiles of the various solar components were fitted by convolution with a Gaussian kernel. This transformation was generally negligible, except for widely discordant resolution (e.g., some SOFIN spectra of Hale-Bopp with too long exposures at too low elevations in the Cassegrain focus). The intensity fit was done by comparing the intensity of the solar photospheric lines in each component.

After removal of the dust, twilight and moonlight spectra, no solar feature subsists. The remaining contamination consists of an extremely weak quasi-featureless continuum due to a residual of improperly subtracted scattered light in the instrument, and to the underlying sky background. This continuum is fitted by a spline through the emission-free intervals of the spectrum and is subtracted from the spectrum.

The signal levels on the CCD amount at best to only a few electrons per pixel above the bias level, except in the major $R$ lines of the abundant species where it is typically $10^{2}$ to $10^{3}$ electrons.

The linearity of the spectrographs is very good, and well maintained. For instance at ESO, the linearity of the UVES detector between 0 and $10^{5} \mathrm{ADU}$ is guaranteed to within about $1 \%$ by the frequent verifications performed by the staff. Whenever possible, however, we checked the linearity directly on our data by comparing cometary lines and stellar spectra appearing in overlapping orders with vastly different sensitivities. The comparison of specific line ratios to their theoretical values yields the same conclusion.

\section{Fluorescence model of $\mathrm{CN}$}

The structure of the $\mathrm{CN}$ radical and the details of the fluorescence calculations are presented elsewhere (Zucconi \& Festou 1985) and will not be repeated here. Instead, we will only focus on some features and on their improvements.

The CN fluorescence is very sensitive to the Swings effect (Swings 1941) and the computation requires accurate line wavelengths and solar fluxes. Wavelengths of the ${ }^{12} \mathrm{C}^{14} \mathrm{~N} \mathrm{~B}-\mathrm{X}$ system (the violet system) have been computed using the spectroscopic constants of Ram et al. (2006), leading to an accuracy better than $1 \mathrm{~m} \AA$. On the other hand we have used the Kurucz atlas (Kurucz 2005) to evaluate the solar intensities in the violet bands.

The influence of the A-X system on the shape of the violet bands cannot be neglected because the pumping via this system also affects the population of the ground state levels.

Unfortunately, we do not have high-resolution absolute spectra of the Sun in the red and near infrared. Ground-based atlases contain many strong water absorption lines and are unsuitable. Hence we decided to use the solar fluxes of Labs \& Neckel (1970). Although the Swings effect is thus ignored in the red bands, we obtain a better fit of the spectra than when using the Kurucz atlas in the red and near infrared. This could be due to the fact that there is a large number of $\mathrm{CN}$ lines in those bands, minimizing the Swings effect. There is no extensive laboratory study of the violet bands of ${ }^{13} \mathrm{C}^{14} \mathrm{~N}$ and ${ }^{12} \mathrm{C}^{15} \mathrm{~N}$ and only the most intense bands have been observed. We derived the isotope line wavelengths from the ${ }^{12} \mathrm{C}^{14} \mathrm{~N}$ lines using the isotopic shift formula. The predicted wavelengths agree with the faint isotope lines observed in our spectra. We also checked that the computed wavelengths agree with the available data for the ground vibrational state (Hübner et al. 2005).

Dipole moment, radiative lifetimes and Franck-Condon factors for the violet bands are those listed in Zucconi \& Festou (1985) and we adjusted the vibrational transition rate and the lifetime $\tau_{\mathrm{A}}$ of the A state to get the best fit of our observations. The value adopted for $\tau_{\mathrm{A}}\left(v^{\prime}=0\right), 10^{-5} \mathrm{~s}$, is very close to the average between various experimental measurements and theoretical estimates (e.g., Lu et al. 1992; Bauschlicher et al. 1988, and references therein). It is also in excellent agreement with the value based on a recent analysis of the solar spectrum (Sauval et al. 2009).

Because we probe the central part of the coma, collisions may play a non negligible role and we had to include this effect in our model. Ideally this would lead to consideration of the density variation in the field of view and the dependence of collisional cross sections as a function of the relative velocity of the colliders and of the rotational quantum number.

The general formula expressing the transition probability between two levels is given by

$g_{l} C_{l \rightarrow u}=g_{u} C_{u \rightarrow l} \exp \left(-\left(E_{u}-E_{l}\right) / k T\right)$

where $u$ and $l$ denote the upper and lower levels of the transition respectively. $g_{u}$ and $g_{l}$ are the statistical weights of the levels and $E_{u}$ and $E_{l}$ are their corresponding energies.

In order to reduce the number of free parameters in the model, we have chosen to use a constant transition probability $Q \equiv C_{u \rightarrow l}$ between two rotational states. We have also assumed that the collisional interactions are mainly dipolar, so that $Q$ is non zero only for $\Delta J=0, \pm 1$. We have found that the best fit is obtained with $Q$ independent of $\Delta J$. It should be noted that the temperature $T$ appearing here is not the thermodynamic temperature of the medium because the $\mathrm{CN}$ radical is not in thermal equilibrium but rather in a statistical equilibrium involving both radiative and collisional processes. $T$ should be taken as a temperature characterizing the velocity distribution of the colliding particles responsible for the rotational excitation of the radicals. It is similar to the kinetic temperatures defined in studies of the interstellar medium or of gaseous nebulae.

The "average" temperatures required to achieve the best agreement between observed and synthetic spectra are appreciably higher than those indicated in the interpretation of radioastronomical spectra (20-100 K near 1 AU from the Sun) of the mother molecules $\mathrm{H}_{2} \mathrm{O}, \mathrm{CH}_{3} \mathrm{OH}, \mathrm{CO}$ in several comets (Bockelée-Morvan \& Crovisier 1987; Bockelée-Morvan et al. 1994; Biver et al. 2002). The difference may be related to the fact that we are dealing here with daughter products, which are formed at greater nucleocentric distances in the coma. We note further that collisions with electrons may play a role in the excitation of the lower rotational levels of $\mathrm{CN}$, as suggested in the case of water by Xie \& Mumma (1992) and of methanol by Bockelée-Morvan et al. (1994). The cross-sections for rotational excitation of $\mathrm{CN}$ by electron impact are indeed quite appreciable at low energies (Allison \& Dalgarno 1971). We note that ions (essentially $\mathrm{H}_{3} \mathrm{O}^{+}$and $\mathrm{H}_{2} \mathrm{O}^{+}$, the most abundant ones) might in principle also contribute to excite $\mathrm{CN}$ rotationally. However, their relevant cross-sections, although expected to be rather high (Xie \& Mumma (1992), are unknown and the corresponding excitation rates would anyhow be appreciably smaller than those of electrons because of the much larger mass of the ions and their consequently $\sim 200$ times lower velocities.

A study under way will take into consideration the structure of the coma, in particular the distributions with nucleocentric distance of the densities, temperatures, and velocities, solving the statistical equilibrium equations point by point, with both radiative and collisional terms. The hope is that some information may then be derived on the nature and physical properties of the different colliders. 


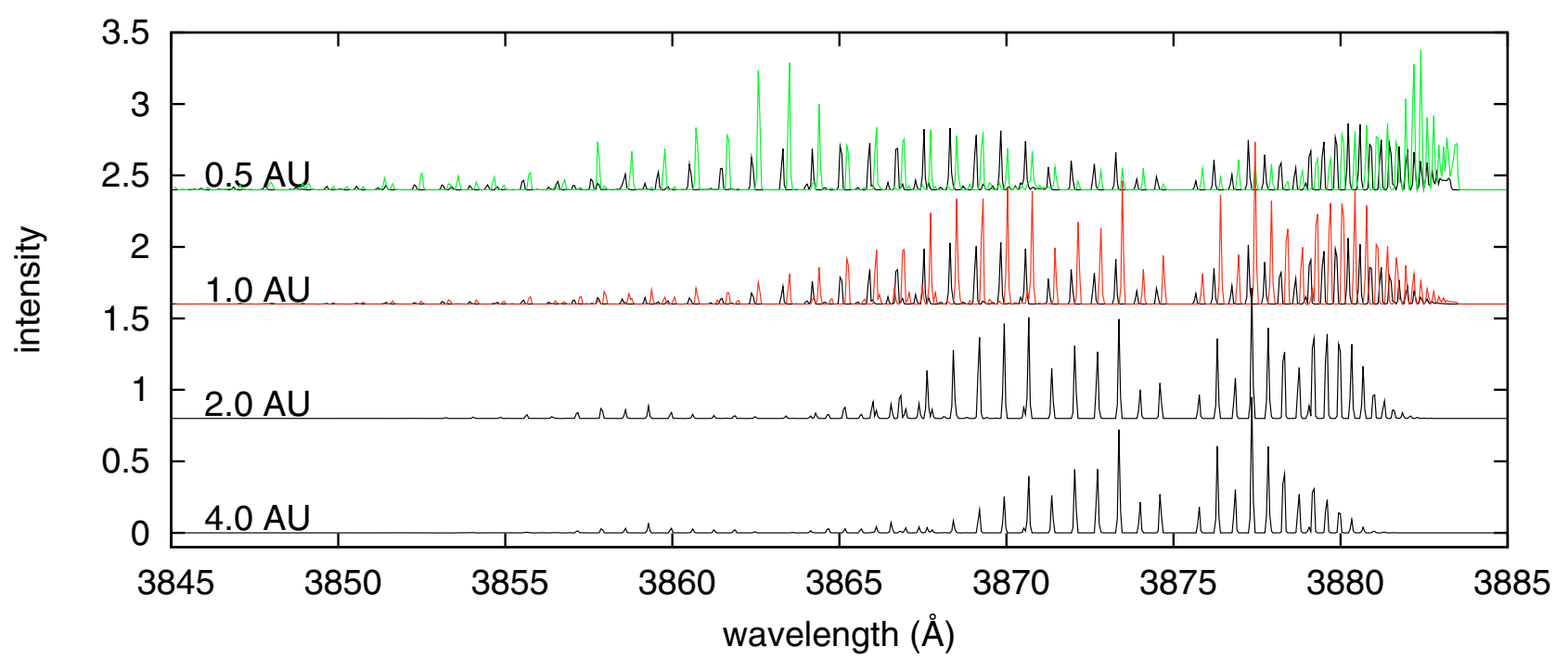

Fig. 3. Synthetic spectra of ${ }^{12} \mathrm{C}^{14} \mathrm{~N}$ at various heliocentric distances. The intensity scale is arbitrary. The $R$-branch of the (0-0) band extends shortward of $3875 \AA$ and the $P$-branch longward of $3875 \AA$. Weak lines of the $P$-branch of the $(1-1)$ band are visible at wavelengths shorter than $3872.5 \AA$ A. The black spectra correspond to a collisionless coma with $\dot{r}=0$. The green spectrum shows the Swings effect for $\dot{r}=20 \mathrm{~km} \mathrm{~s}{ }^{-1}$. The red spectrum corresponds to strong collisional effects $\left(Q=0.01 \mathrm{~s}^{-1}, T=300\right.$ and $\left.\dot{r}=0\right)$. The red and green spectra have been slightly shifted along the wavelength axis for clarity.

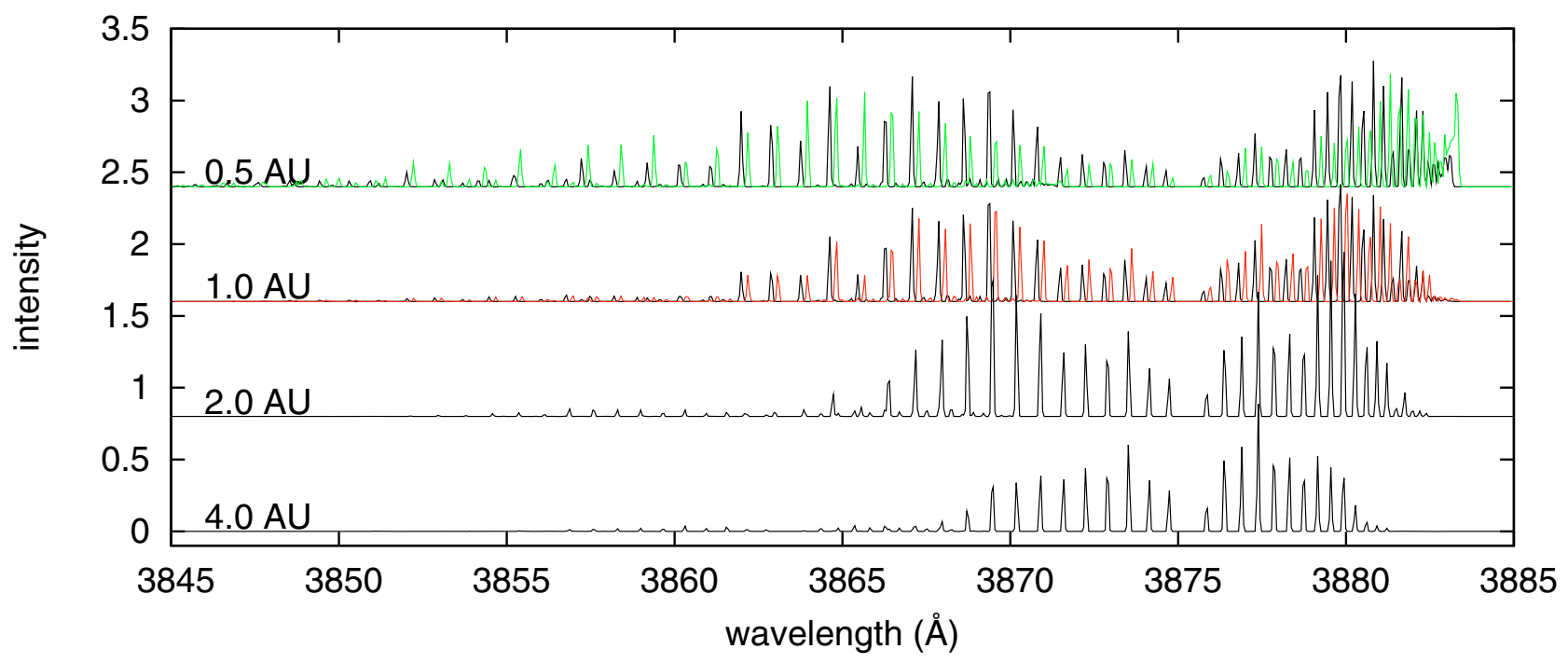

Fig. 4. Same as Fig. 3 for ${ }^{12} \mathrm{C}^{15} \mathrm{~N}$.

The effects of the heliocentric distance $r$ and velocity $\dot{r}$ and of the collisions on the fluorescence spectra of ${ }^{12} \mathrm{C}^{14} \mathrm{~N}$ and ${ }^{12} \mathrm{C}^{15} \mathrm{~N}$ are shown in Figs. 3-5. Synthetic spectra of the various isotopologues ${ }^{12} \mathrm{C}^{14} \mathrm{~N},{ }^{13} \mathrm{C}^{14} \mathrm{~N}$ and ${ }^{12} \mathrm{C}^{15} \mathrm{~N}$ had to be derived for each observing circumstance $(\dot{r}, r)$. The Swings effect shown in Fig. 3 is especially important since the solar photospheric spectrum shows strong $\mathrm{CN}$ absorption lines which reduce considerably the excitation at $\dot{r}=0$ (see also Fig. 5). Because of the wavelength separation between ${ }^{12} \mathrm{C}^{14} \mathrm{~N}$ and ${ }^{12} \mathrm{C}^{15} \mathrm{~N}$ the $\dot{r}=0$ spectrum in Fig. 4 is not so severely depressed. Observing comets close to the perihelion is thus particularly interesting because of the weaker contamination by the lines of ${ }^{12} \mathrm{C}^{14} \mathrm{~N}$.

Ideally a complete $3 \mathrm{D}$ model should have been used, with a range of values for $T$ and $Q$ inside the coma. As a first approximation, we used a single cell model, with only one value for each parameter; this appears to adequately reproduce the spectra. The parameters were derived iteratively, through a minimization procedure over the $T$ and $Q$ parameters, so as to best reproduce the intensity of all observed ${ }^{12} \mathrm{C}^{14} \mathrm{~N}$ lines. The median rms deviation of the ratio between the individual observed and modeled ${ }^{12} \mathrm{C}^{14} \mathrm{~N}$ lines is 0.05 , computed over $\sim 30$ lines. For the best spectra the median rms is 0.02 . The same values of $T$ and $Q$ were used to compute the ${ }^{13} \mathrm{C}^{14} \mathrm{~N}$ and ${ }^{12} \mathrm{C}^{15} \mathrm{~N}$ spectra. We expect the models of the isotopologues to be equally good, assuming that the collisional cross sections of all isotopologues are of the same order.

The adopted values for $T$ and $Q$ are given in Table 8. Figure 6 shows the trend of $T$ with the heliocentric distance. The large 

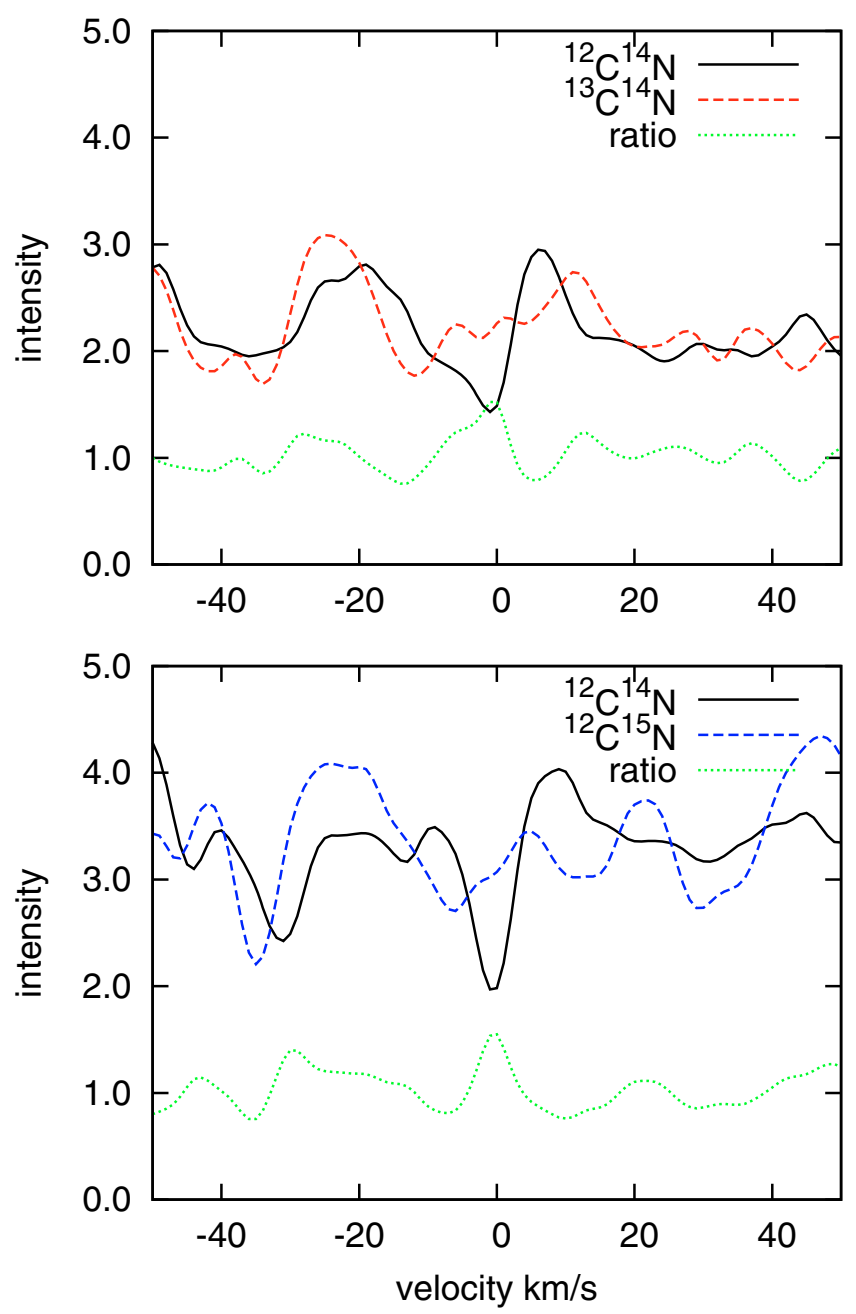

Fig. 5. Swings effect in the $\mathrm{R}$ lines used to estimate the ${ }^{12} \mathrm{C} /{ }^{13} \mathrm{C}$ (top) and ${ }^{14} \mathrm{~N} /{ }^{15} \mathrm{~N}$ ratios. The solid line corresponds to the emission of ${ }^{12} \mathrm{C}^{14} \mathrm{~N}$; the red line to the emission of an equal amount of the rare isotopologue; the green line is the ratio ${ }^{12} \mathrm{C} /{ }^{13} \mathrm{C}$ or ${ }^{14} \mathrm{~N} /{ }^{15} \mathrm{~N}$. Intensity is in arbitrary units. Observing comets close to the perihelion is thus particularly interesting because of the weaker contamination by the lines of ${ }^{12} \mathrm{C}^{14} \mathrm{~N}$.

scatter around $r=1.5 \mathrm{AU}$ is due to the Tempel 1 data. The Deep Impact experiment produced a noticeable but short-lived increase in the collisional excitation temperature.

\section{Analysis}

Very high-resolution synthetic spectra are computed with the IRAF mkldspec task, using the fluorescence model described in Sect. 6. The observed line profile (psf, point spread function) is wider and smoother than the synthetic one and it is possible to find a convolution transforming the latter into the former. The psfmatch task does it, and it is then applied to each spectrum in order to match the synthetic psf of ${ }^{12} \mathrm{C}^{14} \mathrm{~N}$ to the observed instrumental profile for a series of clean lines. The intensities are normalized simultaneously through the same procedure. ${ }^{13} \mathrm{C}^{14} \mathrm{~N}$ and ${ }^{12} \mathrm{C}^{15} \mathrm{~N}$ synthetic spectra are produced by applying the same psfmatch kernel.

The final individual spectra (observed and synthetic) were combined (by comets and observing runs) in small groups (designated by subscript $k$ ) using an optimal weighting scheme in order to maximize the overall signal-to-noise ratio of the observed spectra. This yields a library of observed and synthetic

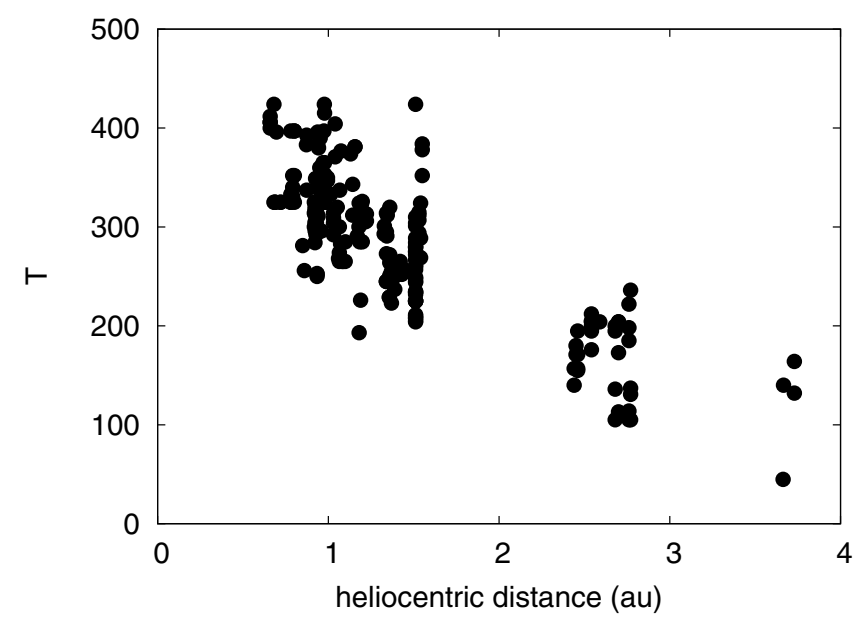

Fig. 6. Variations of the collisional temperature $T$ with the heliocentric distance.

spectra $O_{k}(\lambda)$ and $S_{k, 1}(\lambda)$ (for ${ }^{12} \mathrm{C}^{14} \mathrm{~N}$ ), $S_{k, 2}(\lambda)\left({ }^{13} \mathrm{C}^{14} \mathrm{~N}\right.$ ) and $S_{k, 3}(\lambda)\left({ }^{12} \mathrm{C}^{15} \mathrm{~N}\right)$ - the last two corresponding to isotopic ratios of 1 .

The observed spectrum $O_{k}$ and the synthetic ones $S_{k, i}$ must then be compared in order to obtain the isotopic ratio of the comet corresponding to group $k$. We have to estimate $\alpha_{k}=$ ${ }^{13} \mathrm{C}^{14} \mathrm{~N} /{ }^{12} \mathrm{C}^{14} \mathrm{~N}$ and $\beta_{k}={ }^{12} \mathrm{C}^{15} \mathrm{~N} /{ }^{12} \mathrm{C}^{14} \mathrm{~N}$ such that

$O_{k}(\lambda)=S_{k, 1}(\lambda)+\alpha_{k} S_{k, 2}(\lambda)+\beta_{k} S_{k, 3}(\lambda)$.

This is done in an interactive procedure (see Appendix A).

The values of the isotopic ratios (Table 4) are obtained over the full set of isotopic lines, unblended with ${ }^{12} \mathrm{C}^{14} \mathrm{~N}$. Calculations made with independent subsets helped us to estimate the errors. We also took into account the inaccuracies of the model, which we estimated from the residuals obtained when fitting the ${ }^{12} \mathrm{C}^{14} \mathrm{~N}$ lines. Since the uncertainties are not dominated by random errors, the quoted values are conservative estimates which represent the outer limits beyond which reasonable fits of the rare species cannot be accepted.

Weighted mean values and estimated errors are given in Table 6, using the procedure explained in Appendix B. They are computed for a variety of groups: all data, all data excluding 73P/Schwassmann-Wachmann $3 \mathrm{~B}$ and C, and each of the comet families as defined by Levison (JF, HF, ext, new) or Horner et al. (SP, I, L). There are no significant differences between the groups except perhaps for the JF or $\mathrm{SP}_{\mathrm{III}}$ families which contain - exclusively for $\mathrm{SP}_{\mathrm{III}}$ - the $73 \mathrm{P} /$ Schwassmann-Wachmann 3 fragments. The constancy of the isotopic ratios is interesting in view of the significant differences in molecular abundances exhibited by some of the comets under study (see, e.g., Biver et al. 2006).

Figure 7 shows no correlation of the ratios with the heliocentric distance, confirming our previous results (Manfroid et al. 2005). Now that the $\mathrm{CN}$ and $\mathrm{HCN}$ isotopic ratios are known to be similar (Bockelée-Morvan et al. 2008a), this absence of correlation is no longer a strong argument against $\mathrm{HCN}$ being a major parent of CN. Other possible major parents likely have similar isotopic ratios.

There is also no significant correlation between ${ }^{12} \mathrm{C} /{ }^{13} \mathrm{C}$ and ${ }^{14} \mathrm{~N} /{ }^{15} \mathrm{~N}$ (Fig. 8).

Finally, we looked for possible variations of the isotopic ratios with distance to the nucleus. Such a change would reveal a mixture of $\mathrm{CN}$ parents with different isotopic ratios and different lifetimes (see Table 7). The best spectra for this study are those 
Table 4. Isotopic ratios in $\mathrm{CN}$.

\begin{tabular}{|c|c|c|c|c|}
\hline Runs & Comet & ${ }^{12} \mathrm{C} /{ }^{13} \mathrm{C}$ & ${ }^{14} \mathrm{~N} /{ }^{15} \mathrm{~N}$ & Reference \\
\hline 1 & 122P/de Vico & $90 \pm 10$ & $145 \pm 20$ & Jehin et al. (2004) \\
\hline 2 & C/1996 B2 (Hyakutake) & $\geq 60$ & $\geq 60$ & \\
\hline 3 & C/1996 B2 (Hyakutake) & $\geq 50$ & $\geq 50$ & \\
\hline 4 & C/1995 O1 (Hale-Bopp) & $80 \pm 25$ & $130 \pm 40$ & Manfroid et al. (2005) \\
\hline 5 & C/1995 O1 (Hale-Bopp) & $90 \pm 20$ & $150 \pm 30$ & Manfroid et al. (2005) \\
\hline 6 & C/1995 O1 (Hale-Bopp) & $100 \pm 30$ & $135 \pm 40$ & Manfroid et al. (2005) \\
\hline 7 & 55P/Tempel-Tuttle & - & - & \\
\hline 8 & C/1999 H1 (Lee) & $\geq 60$ & $\geq 60$ & \\
\hline 9,10 & C/1999 S4 (LINEAR) & $90 \pm 30$ & $150 \pm 50$ & Hutsemékers et al. (2005) \\
\hline 11 & C/1999 T1 (McNaught-Hartley) & $80 \pm 20$ & $160 \pm 50$ & \\
\hline 12 & C/2001 A2-A (LINEAR) & $\geq 60$ & $\geq 60$ & \\
\hline 13,14 & C/2000 WM1 (LINEAR) & $100 \pm 20$ & $150 \pm 30$ & Arpigny et al. $(2000,2003)$ \\
\hline 15 & 153P/Ikeya-Zhang & $80 \pm 30$ & $140 \pm 50$ & Jehin et al. (2004) \\
\hline 17,18 & C/2002 X5 (Kudo-Fujikawa) & $90 \pm 20$ & $130 \pm 20$ & \\
\hline 16,19 & C/2002 V1 (NEAT) & $100 \pm 20$ & $160 \pm 35$ & \\
\hline 20 & C/2002 Y1 (Juels-Holvorcem) & $90 \pm 20$ & $150 \pm 35$ & \\
\hline 21 & C/2001 Q4 (NEAT) & $70 \pm 30$ & $130 \pm 40$ & Manfroid et al. (2005) \\
\hline 22,23 & 88P/Howell & $90 \pm 15$ & $140 \pm 20$ & Hutsemékers et al. (2005) \\
\hline 24 & C/2001 Q4 (NEAT) & $90 \pm 15$ & $135 \pm 20$ & Manfroid et al. (2005) \\
\hline $25,28,29$ & C/2002 T7 (LINEAR) & $85 \pm 20$ & $160 \pm 25$ & \\
\hline 26 & C/2003 K4 (LINEAR) & $80 \pm 20$ & $150 \pm 35$ & Manfroid et al. (2005) \\
\hline 30 & C/2003 K4 (LINEAR) & $90 \pm 20$ & $145 \pm 25$ & Manfroid et al. (2005) \\
\hline 31,34 & 9P/Tempel 1 & $110 \pm 20$ & $170 \pm 35$ & Jehin et al. (2006) \\
\hline 32,33 & 9P/Tempel 1 & $95 \pm 15$ & $145 \pm 20$ & Jehin et al. (2006) \\
\hline 35 & 73P-B/Schwassmann-Wachmann 3 & - & - & \\
\hline 36 & 73P-C/Schwassmann-Wachmann 3 & - & - & \\
\hline 37 & 73P-B/Schwassmann-Wachmann 3 & $100 \pm 30$ & $210 \pm 50$ & Jehin et al. (2008) \\
\hline 38,39 & 73P-C/Schwassmann-Wachmann 3 & $100 \pm 20$ & $220 \pm 40$ & Jehin et al. (2008) \\
\hline 40 & C/2006 M4 (SWAN) & $95 \pm 25$ & $145 \pm 50$ & \\
\hline $41-44$ & 17P/Holmes & $90 \pm 20$ & $165 \pm 35$ & \\
\hline $41,43,44$ & 17P/Holmes & $\geq 10$ & $\geq 10$ & \\
\hline 42 & 17P/Holmes & $95 \pm 20$ & $165 \pm 40$ & Bockelée-Morvan et al. (2008a) \\
\hline 45 & 8P/Tuttle & $90 \pm 20$ & $150 \pm 30$ & Bockelée-Morvan et al. (2008b) \\
\hline 46 & C/2007 N3 (Lulin) & $105 \pm 40$ & $150 \pm 50$ & \\
\hline
\end{tabular}

For convenience the data are grouped in observing runs as defined in Table 3. References are given when these are not first determinations, in italics if the values have been revised.
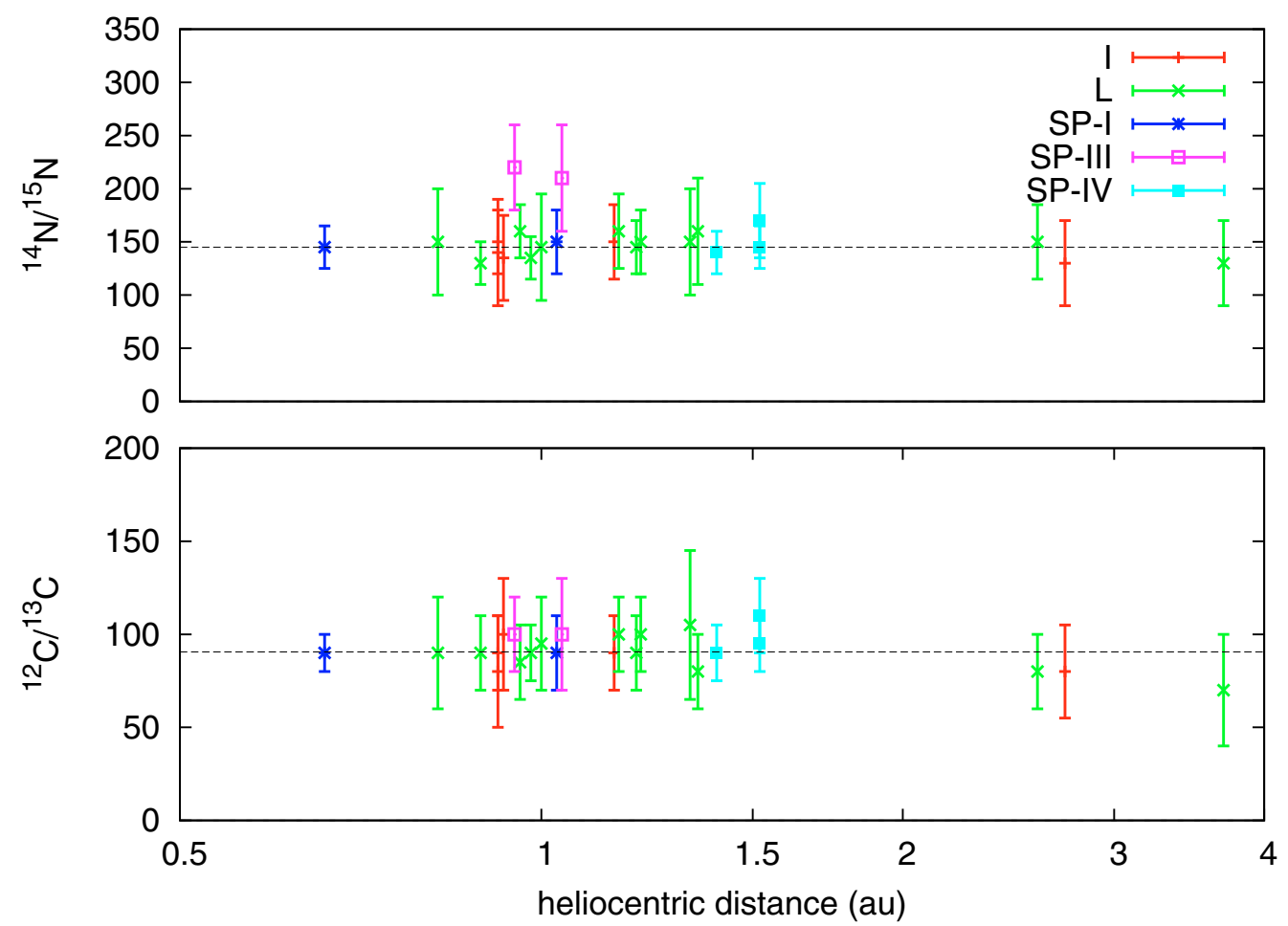

Fig. 7. ${ }^{12} \mathrm{C} /{ }^{13} \mathrm{C}$ and ${ }^{14} \mathrm{~N} /{ }^{15} \mathrm{~N}$ versus the heliocentric distance. The horizontal lines show the mean values (91.0 and 147.8 , respectively). Different symbols refer to different comet families. 


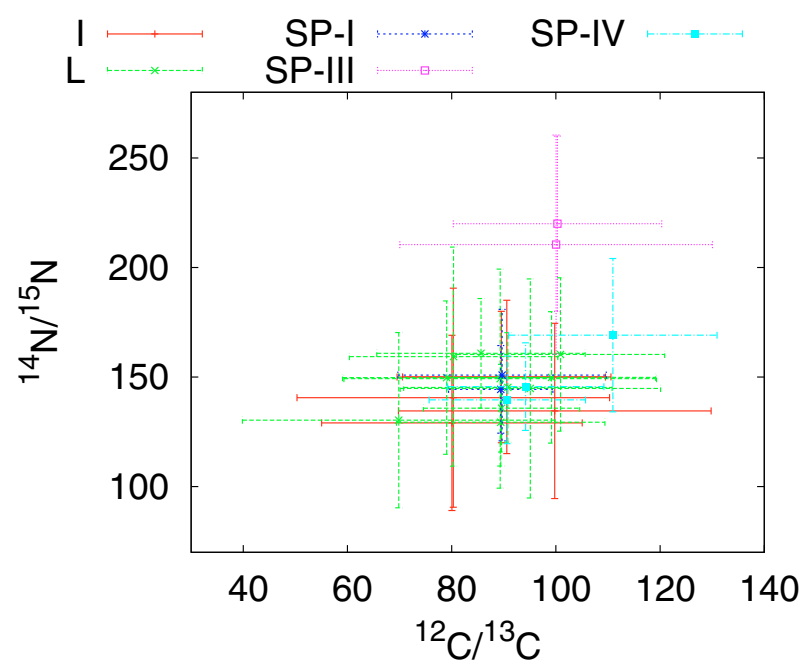

Fig. 8. ${ }^{14} \mathrm{~N} /{ }^{15} \mathrm{~N}$ versus ${ }^{12} \mathrm{C} /{ }^{13} \mathrm{C}$. Different symbols refer to different comet families. Small shifts have been introduced to reduce overlapping.

Table 5. Lines not identified as $\mathrm{CN}$ within the 3857-3880 ̊ range. Most are not yet identified.

\begin{tabular}{ll}
\hline$\lambda \lambda(\AA)$ & Identification \\
\hline 3857.36 & \\
3857.50 & \\
3857.58 & \\
3858.16 & \\
3858.90 & \\
3859.02 & \\
3859.46 & \\
3861.03 & \\
3861.68 & \\
3862.73 & \\
3863.71 & \\
3864.41 & \\
3865.40 & \\
3866.19 & \\
3866.28 & \\
3867.08 & \\
3867.92 & \\
3868.27 & \\
3873.14 & \\
3875.95 & $\mathrm{CH} \mathrm{R}_{1} J^{\prime \prime}=3.5$ \\
3877.09 & \\
3877.20 & \\
3877.47 & $\mathrm{CH} \mathrm{R}_{2} J^{\prime \prime}=1.5$ \\
3877.56 & \\
3877.65 & \\
3878.00 & \\
3878.40 & $\mathrm{CH} \mathrm{R}_{1} J^{\prime \prime}=2.5$ \\
3878.49 & \\
3878.57 & \\
3878.91 & \\
3879.36 & \\
\hline
\end{tabular}

of C/2001 Q4 (NEAT) (run No. 24, 5-7 May 2004). Spectra of sufficient $\mathrm{S} / \mathrm{N}$ ratio were taken at a projected distance of roughly 0,25000 and $50000 \mathrm{~km}$. We found the same isotopic ratios for the three groups. The spatial coverage is relatively small compared to the scale lengths involved, but we could not afford the very long exposure times needed to reach larger distances. Spectra of C/2002 T7 (LINEAR) taken at large nucleocentric distance, but with poorer $\mathrm{S} / \mathrm{N}$ ratios and over a longer time
Table 6. Average isotopic ratios. The weighted mean is computed for a variety of groups: all data, all data excluding $73 \mathrm{P} / \mathrm{Sch}$ wassmannWachmann $3 \mathrm{~B}$ and $\mathrm{C}$, and each of the categories of Levison (JF, HF, EXT, NEW) or Horner et al. (SP, I, L).

\begin{tabular}{lll}
\hline \hline Group & ${ }^{12} \mathrm{C} /{ }^{13} \mathrm{C}$ & ${ }^{14} \mathrm{~N} /{ }^{15} \mathrm{~N}$ \\
\hline All comets & $91.1 \pm 3.7$ & $147.8 \pm 5.6$ \\
All without S-W 3 & $90.6 \pm 3.8$ & $145.2 \pm 5.6$ \\
JF & $97.2 \pm 7.6$ & $156.8 \pm 12.2$ \\
JF without S-W & $96.3 \pm 8.6$ & $146.4 \pm 12.4$ \\
Non JF (Oort) & $89.1 \pm 4.2$ & $144.0 \pm 6.5$ \\
HF & $90.0 \pm 8.4$ & $146.5 \pm 15.1$ \\
EXT & $89.0 \pm 6.9$ & $141.3 \pm 10.7$ \\
NEW & $89.1 \pm 6.8$ & $145.3 \pm 9.5$ \\
SP $_{\text {I }}$ & $90.0 \pm 8.4$ & $146.5 \pm 15.1$ \\
SP $_{\text {III }}$ & $100.0 \pm 15.1$ & $216.1 \pm 27.3$ \\
SP $_{\text {IV }}$ & $96.3 \pm 8.6$ & $146.4 \pm 12.4$ \\
I & $88.2 \pm 9.3$ & $142.8 \pm 14.8$ \\
L & $89.3 \pm 5.8$ & $143.8 \pm 8.1$ \\
\hline
\end{tabular}

Table 7. Isotopic ratios in $\mathrm{CN}$ at various nucleocentric distances. The C/2002 T7 (LINEAR) data include spectra taken at up to $180000 \mathrm{~km}$ but are strongly weighted for the range $24000-45000 \mathrm{~km}$.

\begin{tabular}{llll}
\hline \hline Comet & $\begin{array}{l}\text { Distance } \\
(\mathrm{km})\end{array}$ & ${ }^{12} \mathrm{C} /{ }^{13} \mathrm{C}$ & ${ }^{14} \mathrm{~N} /{ }^{15} \mathrm{~N}$ \\
\hline C/2001 Q4 (NEAT) & $0-3000$ & $90 \pm 20$ & $135 \pm 25$ \\
C/2001 Q4 (NEAT) & 25000 & $95 \pm 20$ & $135 \pm 25$ \\
C/2001 Q4 (NEAT) & 50000 & $90 \pm 20$ & $145 \pm 25$ \\
C/2002 T7 (LINEAR) & $0-2000$ & $85 \pm 20$ & $155 \pm 25$ \\
C/2002 T7 (LINEAR) & $24000-180000$ & $80 \pm 25$ & $165 \pm 30$ \\
\hline
\end{tabular}

interval, show a similar behaviour. Again, the lack of correlation is compatible with $\mathrm{HCN}$ being a major parent of $\mathrm{CN}$, and excludes parents with different isotopic ratios.

The comparison of spectra obtained close to the nucleus and far from it reveals the presence of contaminating emission lines with short scale length in the domain of the B-X $(0,0)$ band. The main ones are listed in Table 5 (see also Fig. 9). Some of them can be attributed to $\mathrm{CH}$, and are within the $P$ branch of $\mathrm{CN}$, i.e., in a region not used for the isotope study, but most are still unidentified. Though an obvious candidate in this domain (Gausset et al. 1965), $\mathrm{C}_{3}$ does not fit the observed spectrum.

Interestingly, one of the brightest ${ }^{12} \mathrm{C}^{15} \mathrm{~N}$ lines (R10), which is blended by an unidentified feature at $\lambda=3867.92 \AA$ close to the nucleus, appears to be useable at larger nucleocentric distances.

\section{Conclusions}

We have derived homogeneously the isotopic ratios ${ }^{12} \mathrm{C} /{ }^{13} \mathrm{C}$ and ${ }^{14} \mathrm{~N} /{ }^{15} \mathrm{~N}$ from high-quality $\mathrm{CN}$ spectra of 18 comets. An improved fluorescence model taking into account the collisional effects has been used. This explains small differences with respect to our previously published values for some of these objects (122P/de Vico, Hale-Bopp, C/1999 S4, C/2000 WM1, C/2003 K4). The new measurements usually agree with our previous estimates. With the possible exception of $73 \mathrm{P} / \mathrm{Sch}$ wassmann-Wachmann 3 , most comets observed so far show the same ${ }^{12} \mathrm{C} /{ }^{13} \mathrm{C}$ and ${ }^{14} \mathrm{~N} /{ }^{15} \mathrm{~N}$ isotopic ratios of the $\mathrm{CN}$ molecule, irrespective of the comet type and the heliocentric distance at which the observations were obtained.

The carbon isotopic ratio appears to be well established. It agrees with the few cometary values determined from HCN 


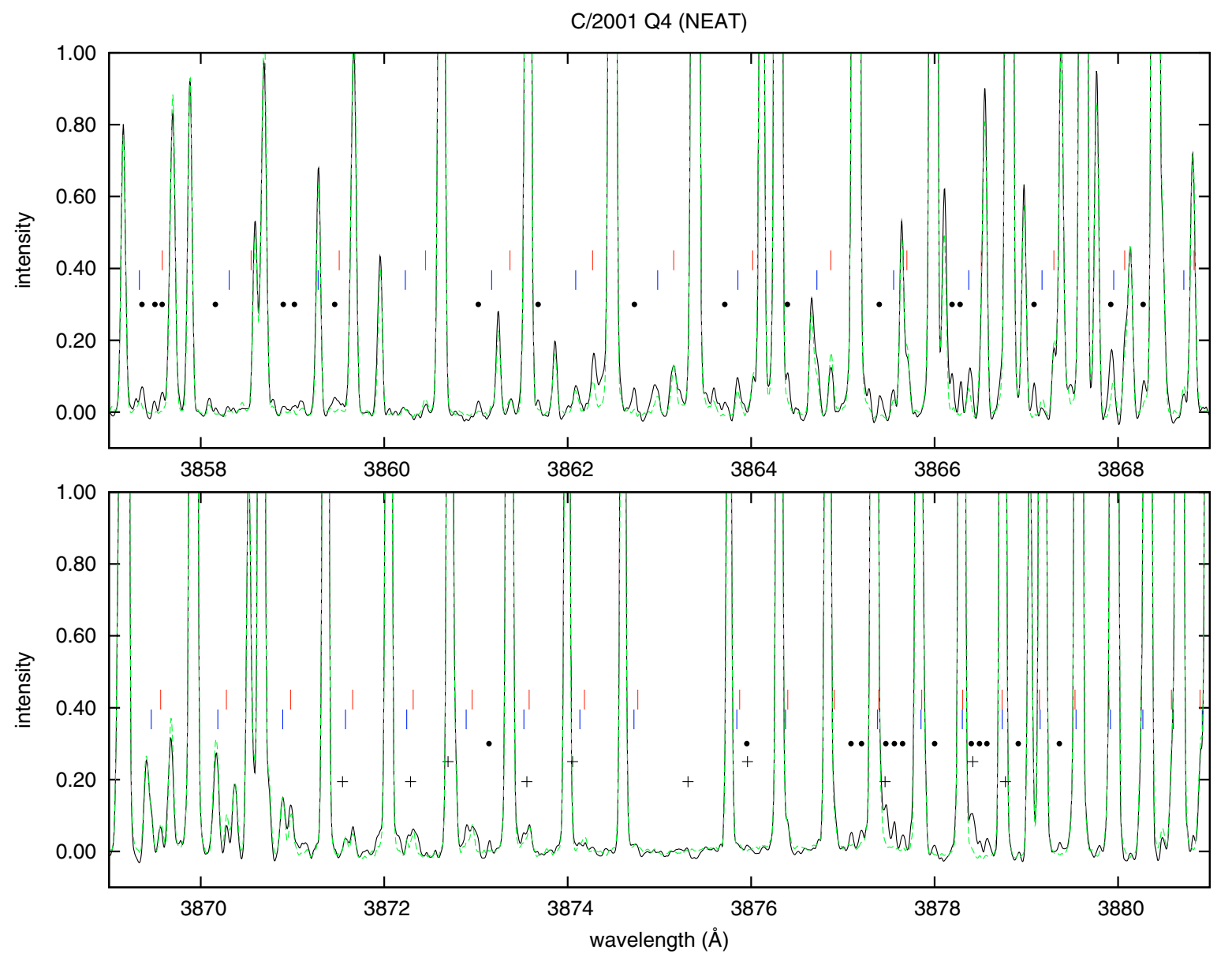

Fig. 9. Spectra of comet C/2001 Q4 (NEAT) obtained close to the nucleus (solid line) and at about $50000 \mathrm{~km}$ (green dashes). The positions of spatially peaked features appearing only in the nucleus spectrum are shown by dots. $\mathrm{CH}$ lines are indicated by + symbols. The upper (red) vertical ticks indicate the position of the main lines of ${ }^{13} \mathrm{C}^{14} \mathrm{~N}$ the lower (blue) ticks indicate the position of the main lines of ${ }^{12} \mathrm{C}^{15} \mathrm{~N}$. The intensity scale is in relative units.

and $\mathrm{C}_{2}$. It is also consistent with the solar system value of 89 (Anders \& Grevesse 1989). It is somewhat higher than the local ISM value $(68 \pm 15$ at galactic radius $R=7.9 \mathrm{kpc}$, Milam et al. 2005 ) and higher still than the value of about 63 at the birth place distance of the Sun at $R=6.6 \mathrm{kpc}$ (Wielen \& Wilson 1997). This can be explained by the galactic ${ }^{13} \mathrm{C}$ enrichment by low mass stars over the last $4.6 \mathrm{Gyr}$. The dispersion of cometary ${ }^{12} \mathrm{C} /{ }^{13} \mathrm{C}$ is small when compared to the large variations observed in $\mathrm{CHON}$ grains of 1P/Halley (Jessberger \& Kissel 1991). On the other hand, dust samples of the Jupiter family comet 81P/Wild 2 collected by Stardust have a solar composition (McKeegan et al. 2006). There must have been little or no fractionation of carbon in the protosolar cloud and in the solar nebula.

The nitrogen ratio in comets is much lower than the Earth atmosphere value (147 vs. 272, Anders \& Grevesse 1989), and the bulk of meteorites. It is even much lower than the primordial value in the solar system (presumed to be that of Jupiter, $435 \pm$ 58, Owen et al. 2001), or the local ISM value (450 \pm 22 at $R=$ $8 \mathrm{kpc}$, Dahmen et al. 1995) or the value of about 415 at the birth place distance of the Sun at $R=6.6 \mathrm{kpc}$ (Wielen \& Wilson 1997).

Primitive meteorites and IDPs are characterized by large ${ }^{15} \mathrm{~N}$ excesses (Floss et al. 2006), as are hotspots in 81P/Wild 2 dust samples (McKeegan et al. 2006). A special mechanism of fractionation (superfractionation) involving interstellar gasphase chemistry in very cold clouds has been proposed as the source of cometary and meteoritic ${ }^{15} \mathrm{~N}$ anomalies (Rodgers \& Charnley 2008) as it can lead to enhancement by a factor of up to 10 .

There is no evidence that the sub-surface material, from which $\mathrm{CN}$ was released in comet 9P/Tempel 1 as a result of the Deep Impact event, was different from the surface material. On the other hand, the 73P/Schwassmann-Wachmann 3 data seem to yield a marginally higher ${ }^{14} \mathrm{~N} /{ }^{15} \mathrm{~N}$ ratio (Jehin et al. 2008). Because of the history of successive fragmentations of 73P/Schwassmann-Wachmann 3, we might have measured recently exposed material. The lower abundance of ${ }^{12} \mathrm{C}^{15} \mathrm{~N}$ may indicate pristine material. However, other peculiarities of $73 \mathrm{P} /$ Schwassmann-Wachmann 3 may point to a unique chemical composition (Dello Russo et al. 2007; Kobayashi et al. 2007). A nuclear spin temperature of about $40 \mathrm{~K}$ for $\mathrm{NH}_{3}$ (Jehin et al. 2008) and $>37 \mathrm{~K}$ for $\mathrm{H}_{2} \mathrm{O}$ (Dello Russo et al. 2007) instead of the more usual $30 \mathrm{~K}$ found in other comets for several molecules (see, e.g., Kawakita 2005) may also indicate a peculiar comet.

Additional measurements of the nitrogen isotopic ratio in $\mathrm{CN}$ and other nitrogen-bearing species in additional comets, especially chemically peculiar ones, are needed to shed more light into these issues.

Acknowledgements. This paper includes data taken at the McDonald Observatory of the University of Texas at Austin, at the W. M. Keck Observatory, which is operated as a scientific partnership by the California Institute of Technology, the University of California, and the National Aeronautics and 
Space Administration, and at the Nordic Optical Telescope (NOT), European Northern Observatory, La Palma, Spain. IRAF is distributed by the National Optical Astronomy Observatory, which is operated by the Association of Universities for Research in Astronomy (AURA) under cooperative agreement with the National Science Foundation.

\section{References}

A'Hearn, M. F., Belton, M. J. S., Delamere, W. A., et al. 2005, Science, 310, 258 Allison, A. C., \& Dalgarno, A. 1971, A\&A, 13, 331

Anders, E., \& Grevesse, N. 1989, Geochim. Cosmochim. Acta, 53, 197

Arpigny, C., Schulz, R., Manfroid, J., et al. 2000, BAAS, 32, 1074

Arpigny, C., Jehin, E., Manfroid, J., et al. 2003, Science, 301, 1522

Bauschlicher, C. W., Jr., Langhoff, S. R., \& Taylor, P. R. 1988, ApJ, 332, 531

Biver, N., Bockelée-Morvan, D., Colom, P., et al. 2002, Earth Moon and Planets, 90,5

Biver, N., Bockelée-Morvan, D., Crovisier, J., et al. 2006, A\&A, 449, 1255

Bockelée-Morvan, D., \& Crovisier, J. 1987, A\&A, 187, 425

Bockelée-Morvan, D., Crovisier, J., Colom, P., \& Despois, D. 1994, A\&A, 287, 647

Bockelée-Morvan, D., Biver, N., Jehin, E., et al. 2008a, A\&A, 679, 249

Bockelée-Morvan, D., Dello Russo, N., Jehin, E., et al. 2008b, LPI Contributions, 1405, 8190

Cochran, A. L., \& Cochran, W. D. 2002, Icarus, 157, 297

Dahmen, G., Wilson, T. L., \& Matteucci, F. 1995, A\&A, 295, 194

Dekker, H., D’Odorico, S., Kaufer, A., Delabre, B., \& Kotzlowski, H. 2000, Proc. SPIE, 4008, 534

Delbouille, L., Roland, G., \& Neven, L. 1973, Atlas photométrique du spectre solaire de 3000 à $10000 \AA$, Univ. Liège

Dello Russo, N.., Vervack, R. J., Weaver, H. A., et al. 2007, Nature, 448, 172

Disanti, M. A., \& Mumma, M. J. 2008, Space Sci. Rev., 70

Floss, C., Stadermann, F. J., Bradley, J. P., et al. 2006, Geochim. Cosmochim. Acta, 70, 2371

Gausset, L., Herzberg, G., Lagerqvist, A., \& Rosen, B. 1965, ApJ, 142, 45

Horner, J., Evans, N. W., Bailey, M. E., \& Asher, D. J. 2003, MNRAS, 343, 1057

Hübner, M., Castillo, M., Davies, P. B., \& Röpcke, J. 2005, Spectrochim. Acta A, 61,57
Hutsemékers, D., Manfroid, J., Jehin, E., et al. 2005, A\&A, 440, L21 Jehin, E., Manfroid, J., Cochran, A. L., et al. 2004, ApJ, 613, L161 Jehin, E., Manfroid, J., Hutsemékers, D., et al. 2006, ApJ, 641, L145 Jehin, E., Manfroid, J., Kawakita, H., et al. 2008, LPI Contributions, 1405, 8319 Jehin, E., Manfroid, J., Hutsemékers, D., et al. 2009, EM\&P, submitted Jessberger, E. K., \& Kissel, J. 1991, IAU Colloq., 116, Comets in the post-Halley era, 167,1075

Jewitt, D. C., Matthews, H. E., Owen, T., \& Meier, R. 1997, Science, 278, 90 Kawakita, H., Watanabe, J., Furusho, R., et al. 2005, ApJ, 623, L49 Kleine, M., Wyckoff, S., Wehinger, P., \& Peterson, B. A. 1995, ApJ, 439, 1021 Kobayashi, H., Kawakita, H., Mumma, M. J., et al. 2007, ApJ, 668, L75

Kurucz, R. L. 2005, Mem. Soc. Astron. It. Suppl., 8, 189

Labs, D., \& Neckel, H. 1970, Sol. Phys., 15, 79

Levison, H. F. 1996, ASPC, 107, 173

Lis, D. C., Keene, J., Young, K., et al. 1997a, Icarus, 130, 355

Lu, R., Huang, Y., \& Halpern, J. B. 1992, ApJ, 395, 710

Manfroid, J., Jehin, E., Hutsemékers, D., et al. 2005, A\&A, 432, L5

McKeegan, K. D., Aléon, J., Bradley, J., et al. 2006, Science, 314, 1724

Meech, K. J., Ageorges, N., A'Hearn, M. F., et al. 2005, Science, 310, 265

Milam, S. N., Savage, C., Brewster, M. A., et al. 2005, ApJ, 634, 1126

Owen, T. 1973, ApJ, 184, 33

Owen, T., Mahaffy, P. R., Niemann, H. B., et al. 2001, ApJ, 553, L77

Ram, R. S., Davis, S. P., Wallace, L., et al. 2006, J. Mol. Spectrosc., 237, 225

Rauer, H., Helbert, J., Arpigny, C., et al. 2003, A\&A, 397, 1109

Rodgers, S. D., \& Charnley, S. B. 2008, MNRAS, 385, L48

Sauval, J., Asplund, M., Grevesse, N., van Hemert, M. C., \& Groenenboom, G. C. 2009, A\&A, to be published

Solc, M., Vanysek, V., \& Kissel, J. 1987, A\&A, 187, 385

Stawikowski, A., \& Greenstein, J. L. 1964, ApJ, 140, 1280

Swings, P. 1941, Lick Obs. Bull., 19, 131

Tull, R. G., MacQueen, P. J., Sneden, C., \& Lambert, D. L. 1995, PASP, 107, 251

Wielen, R., \& Wilson, T. L. 1997, A\&A, 326, 139

Wyckoff, S., \& Wehinger, P. 1988, Rep. Planet. Astron., NASA, 145

Wyckoff, S., Lindholm, E., Wehinger, P. A., et al. 1989, ApJ, 339, 488

Wyckoff, S., Kleine, M., Peterson, B., et al. 2000, ApJ, 535, 991

Xie, X., \& Mumma, M. J. 1992, ApJ, 386, 720

Ziurys, L. M., Savage, C., Brewster, M. A., et al. 1999, ApJ, 527, L67

Zucconi, J. M., \& Festou, M. C. 1985, A\&A, 150, 180 
Table 8. Individual spectra. $r$ is the heliocentric distance in astronomical units (AU), $\Delta$ the geocentric distance, Spectro the spectrograph used, $\mathrm{MJD}=\mathrm{JD}-2400000.5$ the Modified Julian Day, Run the run number, Exp the exposure time in seconds, $R$ the spectral resolution. Slit and Offset give the size of the entrance slit of the spectrograph and the offset from the nucleus. $T$ and $Q$ are the parameters used for the collisional effects in the synthetic spectra.

\begin{tabular}{|c|c|c|c|c|c|c|c|c|c|c|c|c|}
\hline 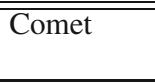 & $\begin{array}{l}r \\
(\mathrm{AU})\end{array}$ & $\begin{array}{l}\Delta \\
(\mathrm{AU})\end{array}$ & $\overline{m_{r}}$ & Spectro & MJD & $\overline{\text { Run }}$ & $\begin{array}{r}\operatorname{Exp} \\
(\mathrm{s}) \\
\end{array}$ & $\overline{\bar{R}}$ & $\begin{array}{r}\text { Slit } \\
\left(1 \times^{\prime \prime}\right)\end{array}$ & $\begin{array}{r}\text { Offset } \\
\left({ }^{\prime \prime}\right)\end{array}$ & $\begin{array}{c}T \\
(\mathrm{~K}) \\
\end{array}$ & $\begin{array}{c}+\log Q \\
\left(\mathrm{~s}^{-1}\right)\end{array}$ \\
\hline deVico & 0.66 & 1.00 & 5.60 & 2DCoudé & 49993.488 & 1 & 600 & 60000 & $1.20 \times 8.20$ & 0 & 410 & 1.9 \\
\hline deVico & 0.66 & 1.00 & 5.60 & 2DCoudé & 49993.502 & 1 & 600 & 60000 & $1.20 \times 8.20$ & 0 & 410 & 1.8 \\
\hline deVico & 0.66 & 0.99 & 5.62 & 2DCoudé & 49994.475 & 1 & 1500 & 60000 & $1.20 \times 8.20$ & 0 & 410 & 1.8 \\
\hline deVico & 0.66 & 0.99 & 5.62 & 2DCoudé & 49994.495 & 1 & 1200 & 60000 & $1.20 \times 8.20$ & 100 & 400 & 1.7 \\
\hline Hyakutake & 1.36 & 0.54 & 6.55 & 2DCoudé & 50151.467 & 2 & 1800 & 60000 & $1.20 \times 8.20$ & 0 & 230 & 2.8 \\
\hline Hyakutake & 1.36 & 0.54 & 6.55 & 2DCoudé & 50151.490 & 2 & 1800 & 60000 & $1.20 \times 8.20$ & 0 & 230 & 2.7 \\
\hline Hyakutake & 0.87 & 0.28 & 4.79 & 2DCoudé & 50175.090 & 3 & 1800 & 200000 & $1.20 \times 8.20$ & 0 & 390 & 3.1 \\
\hline Hyakutake & 0.87 & 0.28 & 4.78 & 2DCoudé & 50175.113 & 3 & 1800 & 200000 & $1.20 \times 8.20$ & 0 & 340 & 1.1 \\
\hline Hyakutake & 0.87 & 0.28 & 4.78 & 2DCoudé & 50175.134 & 3 & 1800 & 200000 & $1.20 \times 8.20$ & 0 & 380 & 3.1 \\
\hline $\mathrm{HB}$ & 2.77 & 3.02 & 3.19 & 2DCoudé & 50367.086 & 4 & 1800 & 60000 & $1.20 \times 8.20$ & 0 & 140 & 1.0 \\
\hline HB & 2.77 & 3.02 & 3.19 & 2DCoudé & 50367.111 & 4 & 1800 & 60000 & $1.20 \times 8.20$ & 0 & 130 & 0.7 \\
\hline HB & 2.77 & 3.02 & 3.19 & 2DCoudé & 50367.141 & 4 & 1800 & 60000 & $1.20 \times 8.20$ & 0 & - & - \\
\hline $\mathrm{HB}$ & 2.77 & 3.02 & 3.19 & 2DCoudé & 50367.165 & 4 & 1800 & 60000 & $1.20 \times 8.20$ & 0 & 240 & 0.8 \\
\hline HB & 2.76 & 3.03 & 3.19 & 2DCoudé & 50368.066 & 4 & 1800 & 60000 & $1.20 \times 8.20$ & 0 & - & - \\
\hline $\mathrm{HB}$ & 2.76 & 3.03 & 3.19 & 2DCoudé & 50368.091 & 4 & 1800 & 60000 & $1.20 \times 8.20$ & 0 & - & - \\
\hline HB & 2.76 & 3.03 & 3.19 & 2DCoudé & 50368.115 & 4 & 1800 & 60000 & $1.20 \times 8.20$ & 0 & 190 & 0.7 \\
\hline HB & 2.76 & 3.03 & 3.19 & 2DCoudé & 50368.138 & 4 & 1800 & 60000 & $1.20 \times 8.20$ & 0 & 110 & 0.1 \\
\hline HB & 2.76 & 3.03 & 3.19 & 2DCoudé & 50368.162 & 4 & 1800 & 60000 & $1.20 \times 8.20$ & 0 & 220 & 0.9 \\
\hline HB & 2.70 & 3.04 & 3.08 & 2DCoudé & 50373.062 & 4 & 1800 & 60000 & $1.20 \times 8.20$ & 0 & - & - \\
\hline HB & 2.70 & 3.04 & 3.08 & 2DCoudé & 50373.086 & 4 & 1800 & 60000 & $1.20 \times 8.20$ & 0 & - & - \\
\hline HB & 2.70 & 3.04 & 3.08 & 2DCoudé & 50373.110 & 4 & 1800 & 60000 & $1.20 \times 8.20$ & 0 & 170 & 1.0 \\
\hline HB & 2.70 & 3.04 & 3.08 & 2DCoudé & 50373.134 & 4 & 1800 & 60000 & $1.20 \times 8.20$ & 0 & 200 & 0.1 \\
\hline HB & 2.68 & 3.04 & 3.08 & 2DCoudé & 50374.057 & 4 & 1800 & 60000 & $1.20 \times 8.20$ & 0 & 200 & 0.8 \\
\hline HB & 2.68 & 3.04 & 3.08 & 2DCoudé & 50374.080 & 4 & 1800 & 60000 & $1.20 \times 8.20$ & 0 & - & - \\
\hline HB & 2.68 & 3.04 & 3.08 & 2DCoudé & 50374.103 & 4 & 1800 & 60000 & $1.20 \times 8.20$ & 0 & - & - \\
\hline HB & 2.68 & 3.04 & 3.08 & 2DCoudé & 50374.126 & 4 & 1800 & 60000 & $1.20 \times 8.20$ & 0 & 140 & 0.9 \\
\hline HB & 0.92 & 1.40 & -1.12 & 2DCoudé & 50544.068 & 5 & 120 & 200000 & $1.20 \times 8.20$ & 0 & 300 & 3.2 \\
\hline HB & 0.92 & 1.40 & -1.12 & 2DCoudé & 50544.079 & 5 & 900 & 200000 & $1.20 \times 8.20$ & 0 & 310 & 1.9 \\
\hline HB & 0.92 & 1.40 & -1.12 & 2DCoudé & 50544.112 & 5 & 1800 & 200000 & $1.20 \times 8.20$ & 0 & - & - \\
\hline HB & 0.92 & 1.42 & -1.15 & SOFIN & 50545.863 & 6 & 1814 & 70000 & $0.45 \times 3.80$ & 0 & 280 & 3.0 \\
\hline HB & 0.92 & 1.42 & -1.15 & SOFIN & 50545.886 & 6 & 2106 & 70000 & $0.45 \times 3.80$ & 0 & 320 & 3.2 \\
\hline HB & 0.92 & 1.43 & -1.17 & SOFIN & 50546.832 & 6 & 600 & 70000 & $0.45 \times 3.80$ & 0 & 300 & 3.0 \\
\hline HB & 0.92 & 1.43 & -1.17 & SOFIN & 50546.839 & 6 & 460 & 70000 & $0.45 \times 3.80$ & 0 & 300 & 3.2 \\
\hline HB & 0.92 & 1.43 & -1.17 & SOFIN & 50546.865 & 6 & 3838 & 70000 & $0.45 \times 3.80$ & 0 & 310 & 2.9 \\
\hline HB & 0.93 & 1.44 & -1.19 & SOFIN & 50547.836 & 6 & 1800 & 70000 & $0.45 \times 3.80$ & 0 & 300 & 2.9 \\
\hline HB & 0.93 & 1.44 & -1.19 & SOFIN & 50547.862 & 6 & 1800 & 70000 & $0.45 \times 3.80$ & 0 & 300 & 3.2 \\
\hline $\mathrm{HB}$ & 0.93 & 1.44 & -1.19 & SOFIN & 50547.887 & 6 & 1800 & 70000 & $0.45 \times 3.80$ & 0 & 290 & 3.0 \\
\hline HB & 0.93 & 1.45 & -1.20 & SOFIN & 50548.837 & 6 & 1200 & 70000 & $0.45 \times 3.80$ & 0 & 320 & 3.1 \\
\hline HB & 0.93 & 1.45 & -1.20 & SOFIN & 50548.856 & 6 & 1200 & 70000 & $0.45 \times 3.80$ & 0 & 320 & 3.2 \\
\hline HB & 0.93 & 1.45 & -1.20 & SOFIN & 50548.874 & 6 & 1200 & 70000 & $0.45 \times 3.80$ & 0 & 300 & 3.1 \\
\hline HB & 0.93 & 1.45 & -1.10 & SOFIN & 50548.893 & 6 & 1200 & 70000 & $0.45 \times 3.80$ & 0 & 320 & 3.0 \\
\hline HB & 0.93 & 1.47 & -1.12 & SOFIN & 50549.837 & 6 & 1200 & 70000 & $0.45 \times 3.80$ & 0 & 250 & 2.7 \\
\hline $\mathrm{HB}$ & 0.93 & 1.47 & -1.12 & SOFIN & 50549.855 & 6 & 1200 & 70000 & $0.45 \times 3.80$ & 0 & 250 & 2.8 \\
\hline HB & 0.93 & 1.47 & -1.12 & SOFIN & 50549.875 & 6 & 1200 & 70000 & $0.45 \times 3.80$ & 0 & 250 & 3.0 \\
\hline HB & 0.94 & 1.48 & -1.14 & SOFIN & 50550.836 & 6 & 1200 & 70000 & $0.45 \times 3.80$ & 0 & 300 & 3.0 \\
\hline HB & 0.94 & 1.48 & -1.14 & SOFIN & 50550.857 & 6 & 1516 & 70000 & $0.45 \times 3.80$ & 0 & 300 & 2.6 \\
\hline HB & 0.94 & 1.48 & -1.14 & SOFIN & 50550.877 & 6 & 1200 & 70000 & $0.45 \times 3.80$ & 0 & 400 & 3.2 \\
\hline HB & 0.94 & 1.48 & -1.14 & SOFIN & 50550.896 & 6 & 1199 & 70000 & $0.45 \times 3.80$ & 0 & 380 & 3.0 \\
\hline HB & 0.94 & 1.49 & -1.16 & SOFIN & 50551.834 & 6 & 1200 & 70000 & $0.45 \times 3.80$ & 0 & 390 & 2.2 \\
\hline HB & 0.94 & 1.49 & -1.16 & SOFIN & 50551.856 & 6 & 1200 & 70000 & $0.45 \times 3.80$ & 0 & 380 & 3.2 \\
\hline HB & 0.94 & 1.49 & -1.16 & SOFIN & 50551.874 & 6 & 1199 & 70000 & $0.45 \times 3.80$ & 0 & 350 & 2.7 \\
\hline HB & 0.94 & 1.49 & -1.16 & SOFIN & 50551.892 & 6 & 1200 & 70000 & $0.45 \times 3.80$ & 0 & 340 & 3.0 \\
\hline TT & 1.19 & 0.36 & 10.24 & 2DCoudé & 50830.104 & 7 & 1800 & 60000 & $1.20 \times 8.20$ & 0 & 230 & 1.7 \\
\hline TT & 1.18 & 0.36 & 10.23 & 2DCoudé & 50831.158 & 7 & 1800 & 60000 & $1.20 \times 8.20$ & 0 & 190 & 1.9 \\
\hline Lee & 1.54 & 0.85 & 8.74 & 2DCoudé & 51444.219 & 8 & 1800 & 60000 & $1.20 \times 8.20$ & 0 & 270 & 1.2 \\
\hline 1999S4 & 0.97 & 1.19 & 7.81 & 2DCoudé & 51720.377 & 9 & 1800 & 60000 & $1.20 \times 8.20$ & 0 & 340 & 1.9 \\
\hline 1999S4 & 0.96 & 1.16 & 7.87 & 2DCoudé & 51721.362 & 9 & 1800 & 60000 & $1.20 \times 8.20$ & 0 & 300 & 1.9 \\
\hline 1999S4 & 0.86 & 0.80 & 8.39 & 2DCoudé & 51731.387 & 10 & 1800 & 60000 & $1.20 \times 8.20$ & 0 & 260 & 2.0 \\
\hline
\end{tabular}


Table 8. continued.

\begin{tabular}{|c|c|c|c|c|c|c|c|c|c|c|c|c|}
\hline$\overline{\text { Comet }}$ & $\begin{array}{l}r \\
(\mathrm{AU})\end{array}$ & $\begin{array}{l}\Delta \\
(\mathrm{AU})\end{array}$ & $m_{r}$ & $\overline{\text { Spectro }}$ & MJD & $\overline{\text { Run }}$ & $\begin{array}{r}\operatorname{Exp} \\
(\mathrm{s})\end{array}$ & $\overline{\bar{R}}$ & $\begin{array}{r}\text { Slit } \\
\left({ }^{\prime \prime} \times^{\prime \prime}\right)\end{array}$ & $\begin{array}{r}\text { Offset } \\
\left({ }^{\prime \prime}\right)\end{array}$ & $\begin{array}{c}T \\
(\mathrm{~K})\end{array}$ & $\begin{array}{c}5+\log Q \\
\left(\mathrm{~s}^{-1}\right)\end{array}$ \\
\hline 1999S4 & 0.85 & 0.76 & 8.50 & 2DCoudé & 51732.390 & 10 & 1800 & 60000 & $1.20 \times 8.20$ & 0 & 280 & 1.9 \\
\hline 1999S4 & 0.80 & 0.52 & 8.91 & 2DCoudé & 51739.388 & 10 & 1800 & 60000 & $1.20 \times 8.20$ & 0 & 400 & 1.7 \\
\hline 1999S4 & 0.80 & 0.52 & 8.91 & 2DCoudé & 51739.414 & 10 & 1800 & 60000 & $1.20 \times 8.20$ & 0 & - & - \\
\hline 1999S4 & 0.80 & 0.52 & 8.91 & 2DCoudé & 51739.439 & 10 & 1800 & 60000 & $1.20 \times 8.20$ & 0 & 350 & 1.1 \\
\hline 1999S4 & 0.80 & 0.52 & 8.91 & 2DCoudé & 51739.459 & 10 & 1200 & 60000 & $1.20 \times 8.20$ & 0 & 400 & 1.7 \\
\hline 1999S4 & 0.79 & 0.49 & 8.84 & 2DCoudé & 51740.423 & 10 & 1200 & 60000 & $1.20 \times 8.20$ & 0 & 330 & 0.1 \\
\hline 1999S4 & 0.79 & 0.46 & 8.86 & 2DCoudé & 51741.443 & 10 & 1800 & 60000 & $1.20 \times 8.20$ & 0 & 340 & 1.2 \\
\hline 1999S4 & 0.79 & 0.46 & 8.86 & 2DCoudé & 51741.463 & 10 & 1200 & 60000 & $1.20 \times 8.20$ & 0 & 350 & 1.0 \\
\hline 1999S4 & 0.78 & 0.44 & 8.87 & 2DCoudé & 51742.359 & 10 & 1800 & 60000 & $1.20 \times 8.20$ & 0 & 330 & 1.1 \\
\hline 1999S4 & 0.78 & 0.44 & 8.87 & 2DCoudé & 51742.384 & 10 & 1800 & 60000 & $1.20 \times 8.20$ & 0 & 330 & 0.9 \\
\hline 1999S4 & 0.78 & 0.44 & 8.87 & 2DCoudé & 51742.419 & 10 & 1800 & 60000 & $1.20 \times 8.20$ & 0 & 400 & 0.8 \\
\hline 1999S4 & 0.78 & 0.44 & 8.87 & 2DCoudé & 51742.443 & 10 & 1800 & 60000 & $1.20 \times 8.20$ & 0 & 330 & 0.1 \\
\hline 1999S4 & 0.78 & 0.44 & 8.88 & 2DCoudé & 51742.463 & 10 & 1200 & 60000 & $1.20 \times 8.20$ & 0 & 330 & 1.2 \\
\hline 1999T1 & 1.34 & 1.31 & 7.11 & 2DCoudé & 51932.520 & 11 & 1200 & 60000 & $1.20 \times 8.20$ & 0 & - & - \\
\hline 1999T1 & 1.34 & 1.31 & 7.11 & 2DCoudé & 51932.537 & 11 & 1200 & 60000 & $1.20 \times 8.20$ & 0 & - & - \\
\hline 1999T1 & 1.34 & 1.30 & 7.12 & 2DCoudé & 51933.449 & 11 & 1800 & 60000 & $1.20 \times 8.20$ & 0 & 300 & 2.0 \\
\hline 1999T1 & 1.34 & 1.30 & 7.12 & 2DCoudé & 51933.476 & 11 & 1800 & 60000 & $1.20 \times 8.20$ & 0 & 310 & 1.1 \\
\hline 1999T1 & 1.34 & 1.30 & 7.12 & 2DCoudé & 51933.505 & 11 & 1800 & 60000 & $1.20 \times 8.20$ & 0 & - & - \\
\hline 1999T1 & 1.34 & 1.30 & 7.12 & 2DCoudé & 51933.549 & 11 & 700 & 60000 & $1.20 \times 8.20$ & 0 & 270 & 1.9 \\
\hline 1999T1 & 1.36 & 1.30 & 7.13 & 2DCoudé & 51935.439 & 11 & 1800 & 60000 & $1.20 \times 8.20$ & 0 & - & - \\
\hline 1999T1 & 1.36 & 1.30 & 7.13 & 2DCoudé & 51935.468 & 11 & 1800 & 60000 & $1.20 \times 8.20$ & 0 & 320 & 0.9 \\
\hline 1999T1 & 1.36 & 1.30 & 7.13 & 2DCoudé & 51935.494 & 11 & 1800 & 60000 & $1.20 \times 8.20$ & 0 & 270 & 1.9 \\
\hline 1999T1 & 1.36 & 1.30 & 7.13 & 2DCoudé & 51935.522 & 11 & 1800 & 60000 & $1.20 \times 8.20$ & 0 & 250 & -0.4 \\
\hline 1999T1 & 1.36 & 1.30 & 7.13 & 2DCoudé & 51935.545 & 11 & 700 & 60000 & $1.20 \times 8.20$ & 0 & 260 & 1.9 \\
\hline 2001A2-A & 1.36 & 0.43 & 8.45 & 2DCoudé & 52114.341 & 12 & 1800 & 60000 & $1.20 \times 8.20$ & 0 & 270 & 1.8 \\
\hline 2000WM1 & 1.08 & 1.24 & 6.83 & UVES & 52340.362 & 13 & 1550 & 80000 & $0.45 \times 8.00$ & 10 & 270 & 0.1 \\
\hline 2000WM1 & 1.08 & 1.24 & 6.83 & UVES & 52340.381 & 13 & 1550 & 80000 & $0.45 \times 8.00$ & 10 & 270 & 0.1 \\
\hline 2000WM1 & 1.10 & 1.24 & 6.83 & UVES & 52341.368 & 13 & 1550 & 80000 & $0.45 \times 8.00$ & 10 & 270 & 0.1 \\
\hline 2000WM1 & 1.10 & 1.24 & 6.83 & UVES & 52341.387 & 13 & 1550 & 80000 & $0.45 \times 8.00$ & 6 & 290 & 0.1 \\
\hline 2000WM1 & 1.33 & 1.24 & 8.43 & UVES & 52355.362 & 14 & 1550 & 80000 & $0.45 \times 8.00$ & 2 & 290 & 1.0 \\
\hline 2000WM1 & 1.33 & 1.24 & 8.43 & UVES & 52355.380 & 14 & 1550 & 80000 & $0.45 \times 8.00$ & 2 & 300 & 1.0 \\
\hline 2000WM1 & 1.34 & 1.24 & 8.43 & UVES & 52356.363 & 14 & 1610 & 80000 & $0.45 \times 8.00$ & 1 & 290 & 1.1 \\
\hline 2000WM1 & 1.34 & 1.24 & 8.43 & UVES & 52356.382 & 14 & 1610 & 80000 & $0.45 \times 8.00$ & 1 & 310 & 1.0 \\
\hline IZ & 0.92 & 0.42 & 6.39 & 2DCoudé & 52386.431 & 15 & 1200 & 60000 & $1.20 \times 8.20$ & 0 & 310 & 2.1 \\
\hline IZ & 0.92 & 0.42 & 6.39 & 2DCoudé & 52386.447 & 15 & 1200 & 60000 & $1.20 \times 8.20$ & 0 & 310 & 1.2 \\
\hline IZ & 0.92 & 0.42 & 6.39 & 2DCoudé & 52386.464 & 15 & 1200 & 60000 & $1.20 \times 8.20$ & 52 & 320 & 1.9 \\
\hline $2002 \times 5$ & 0.70 & 0.87 & 4.81 & UVES & 52689.013 & 17 & 2000 & 80000 & $0.45 \times 8.00$ & 0 & 400 & 1.9 \\
\hline $2002 \times 5$ & 0.72 & 0.86 & 4.81 & UVES & 52690.007 & 17 & 1100 & 80000 & $0.45 \times 12.00$ & 20 & 330 & 1.2 \\
\hline $2002 \times 5$ & 0.72 & 0.86 & 4.81 & UVES & 52690.020 & 17 & 2000 & 80000 & $0.45 \times 12.00$ & 20 & 330 & 1.2 \\
\hline $2002 \times 5$ & 1.06 & 0.99 & 9.32 & UVES & 52705.017 & 18 & 1800 & 80000 & $0.45 \times 8.00$ & 0 & 300 & 1.5 \\
\hline $2002 \times 5$ & 1.06 & 0.99 & 9.32 & UVES & 52705.039 & 18 & 1800 & 80000 & $0.45 \times 8.00$ & 3 & 270 & 1.2 \\
\hline $2002 \times 5$ & 1.06 & 0.99 & 9.32 & UVES & 52705.060 & 18 & 1800 & 80000 & $0.45 \times 8.00$ & 3 & 270 & 1.2 \\
\hline 2002V1 & 1.22 & 0.83 & 7.89 & UVES & 52647.037 & 16 & 2100 & 80000 & $0.45 \times 8.00$ & 0 & 310 & 1.2 \\
\hline 2002V1 & 1.22 & 0.83 & 7.89 & UVES & 52647.062 & 16 & 2100 & 80000 & $0.45 \times 8.00$ & 0 & 310 & 1.2 \\
\hline 2002V1 & 1.18 & 0.84 & 7.87 & UVES & 52649.031 & 16 & 2100 & 80000 & $0.45 \times 8.00$ & 0 & 320 & 1.2 \\
\hline 2002V1 & 1.18 & 0.84 & 7.87 & UVES & 52649.056 & 16 & 1983 & 80000 & $0.45 \times 8.00$ & 0 & 300 & 1.2 \\
\hline $2002 \mathrm{~V} 1$ & 1.01 & 1.60 & 5.47 & UVES & 52719.985 & 19 & 600 & 80000 & $0.45 \times 8.00$ & 0 & 330 & 1.9 \\
\hline 2002Y1 & 1.14 & 1.56 & 7.13 & UVES & 52788.394 & 20 & 1800 & 80000 & $0.40 \times 8.00$ & 0 & 310 & 1.2 \\
\hline 2002Y1 & 1.14 & 1.56 & 7.13 & UVES & 52788.416 & 20 & 1800 & 80000 & $0.40 \times 8.00$ & 0 & 340 & 1.6 \\
\hline 2002Y1 & 1.16 & 1.55 & 7.14 & UVES & 52789.393 & 20 & 1800 & 80000 & $0.40 \times 8.00$ & 3 & 380 & 1.2 \\
\hline 2002Y1 & 1.16 & 1.55 & 7.14 & UVES & 52789.415 & 20 & 1800 & 80000 & $0.40 \times 8.00$ & 3 & 380 & 1.1 \\
\hline Howell & 1.37 & 1.68 & 11.07 & UVES & 53113.372 & 22 & 3600 & 80000 & $0.44 \times 8.00$ & 0 & 260 & 1.0 \\
\hline Howell & 1.37 & 1.67 & 11.08 & UVES & 53114.364 & 22 & 3600 & 80000 & $0.44 \times 8.00$ & 0 & 250 & 1.2 \\
\hline Howell & 1.37 & 1.67 & 8.78 & UVES & 53115.368 & 22 & 3600 & 80000 & $0.44 \times 8.00$ & 0 & 220 & 1.0 \\
\hline Howell & 1.37 & 1.67 & 8.79 & UVES & 53117.371 & 22 & 3600 & 80000 & $0.44 \times 8.00$ & 0 & 260 & 1.1 \\
\hline Howell & 1.38 & 1.65 & 8.81 & UVES & 53127.372 & 23 & 3600 & 80000 & $0.44 \times 8.00$ & 0 & 260 & 1.2 \\
\hline Howell & 1.39 & 1.65 & 8.81 & UVES & 53128.363 & 23 & 3600 & 80000 & $0.44 \times 8.00$ & 0 & 250 & 1.2 \\
\hline Howell & 1.39 & 1.64 & 8.82 & UVES & 53129.372 & 23 & 3600 & 80000 & $0.44 \times 8.00$ & 0 & 240 & 1.2 \\
\hline Howell & 1.42 & 1.63 & 8.84 & UVES & 53142.358 & 29 & 3600 & 80000 & $0.44 \times 8.00$ & 0 & 270 & 1.2 \\
\hline Howell & 1.43 & 1.62 & 8.95 & UVES & 53146.371 & 29 & 3600 & 80000 & $0.44 \times 8.00$ & 0 & 250 & 1.2 \\
\hline Howell & 1.44 & 1.62 & 8.95 & UVES & 53147.391 & 29 & 2499 & 80000 & $0.44 \times 8.00$ & 0 & 260 & 1.2 \\
\hline Howell & 1.44 & 1.62 & 8.95 & UVES & 53149.361 & 29 & 4900 & 80000 & $0.44 \times 8.00$ & 0 & 260 & 1.2 \\
\hline $2002 \mathrm{~T} 7$ & 0.68 & 0.61 & 4.87 & UVES & 53131.406 & 25 & 1080 & 80000 & $0.44 \times 8.00$ & 5 & 330 & 1.2 \\
\hline $2002 \mathrm{~T} 7$ & 0.68 & 0.61 & 4.87 & UVES & 53131.421 & 25 & 1080 & 80000 & $0.44 \times 8.00$ & 5 & 420 & 1.8 \\
\hline
\end{tabular}


Table 8. continued.

\begin{tabular}{|c|c|c|c|c|c|c|c|c|c|c|c|c|}
\hline$\overline{\text { Comet }}$ & $\begin{array}{l}r \\
(\mathrm{AU})\end{array}$ & $\begin{array}{l}\Delta \\
(\mathrm{AU})\end{array}$ & $m_{r}$ & Spectro & MJD & $\overline{\text { Run }}$ & $\begin{array}{r}\operatorname{Exp} \\
(\mathrm{s})\end{array}$ & $R$ & $\begin{array}{r}\text { Slit } \\
\left({ }^{\prime \prime} \times^{\prime \prime}\right)\end{array}$ & $\begin{array}{r}\text { Offset } \\
\left({ }^{\prime \prime}\right)\end{array}$ & $\begin{array}{c}T \\
(\mathrm{~K})\end{array}$ & $\begin{array}{c}5+\log Q \\
\left(\mathrm{~s}^{-1}\right)\end{array}$ \\
\hline $2002 \mathrm{~T} 7$ & 0.69 & 0.57 & 5.02 & UVES & 53132.412 & 28 & 800 & 80000 & $0.44 \times 8.00$ & 110 & - & - \\
\hline $2002 \mathrm{~T} 7$ & 0.69 & 0.57 & 5.02 & UVES & 53132.424 & 28 & 800 & 80000 & $0.44 \times 8.00$ & 110 & - & - \\
\hline $2002 \mathrm{~T} 7$ & 0.93 & 0.38 & 5.18 & UVES & 53150.983 & 30 & 3208 & 80000 & $0.44 \times 8.00$ & 0 & 350 & 2.1 \\
\hline $2002 \mathrm{~T} 7$ & 0.94 & 0.41 & 5.01 & UVES & 53151.976 & 30 & 2677 & 80000 & $0.40 \times 8.00$ & 0 & 310 & 2.6 \\
\hline $2002 \mathrm{~T} 7$ & 0.94 & 0.42 & 5.00 & UVES & 53152.036 & 30 & 1800 & 80000 & $0.40 \times 8.00$ & 0 & 340 & 2.1 \\
\hline $2002 \mathrm{~T} 7$ & 0.96 & 0.45 & 4.84 & UVES & 53152.970 & 30 & 3900 & 80000 & $0.44 \times 8.00$ & 70 & 330 & 1.1 \\
\hline $2002 \mathrm{~T} 7$ & 0.97 & 0.48 & 4.79 & UVES & 53153.973 & 30 & 487 & 80000 & $0.40 \times 8.00$ & 70 & 370 & 0.6 \\
\hline $2002 \mathrm{~T} 7$ & 0.97 & 0.48 & 4.78 & UVES & 53153.986 & 30 & 3600 & 80000 & $0.44 \times 8.00$ & 70 & 340 & 0.9 \\
\hline $2002 \mathrm{~T} 7$ & 1.17 & 0.95 & 6.10 & UVES & 53166.967 & 31 & 3600 & 80000 & $0.44 \times 8.00$ & 70 & 290 & 1.1 \\
\hline $2002 \mathrm{~T} 7$ & 1.18 & 0.99 & 6.12 & UVES & 53167.967 & 31 & 3000 & 80000 & $0.44 \times 8.00$ & 245 & 310 & 0.9 \\
\hline $2002 \mathrm{~T} 7$ & 1.18 & 0.99 & 6.11 & UVES & 53168.012 & 31 & 3000 & 80000 & $0.44 \times 8.00$ & 245 & 290 & 0.1 \\
\hline $2002 \mathrm{~T} 7$ & 1.20 & 1.03 & 6.24 & UVES & 53168.983 & 31 & 3120 & 80000 & $0.44 \times 8.00$ & 245 & 290 & 0.1 \\
\hline $2002 \mathrm{~T} 7$ & 1.22 & 1.07 & 6.36 & UVES & 53169.983 & 31 & 3000 & 80000 & $0.44 \times 8.00$ & 245 & 310 & -0.3 \\
\hline 2001Q4 & 3.73 & 3.45 & 9.51 & UVES & 52883.293 & 21 & 4500 & 80000 & $0.45 \times 8.00$ & 0 & 160 & 0.1 \\
\hline 2001Q4 & 3.73 & 3.45 & 9.51 & UVES & 52883.349 & 21 & 4500 & 80000 & $0.45 \times 8.00$ & 0 & 130 & 0.7 \\
\hline 2001Q4 & 3.67 & 3.37 & 9.56 & UVES & 52889.236 & 21 & 7200 & 80000 & $0.45 \times 8.00$ & 0 & 140 & 0.8 \\
\hline 2001Q4 & 3.66 & 3.37 & 9.56 & UVES & 52889.320 & 21 & 7200 & 80000 & $0.45 \times 8.00$ & 0 & - & - \\
\hline 2001Q4 & 0.98 & 0.32 & 6.06 & UVES & 53130.958 & 24 & 120 & 80000 & $0.44 \times 8.00$ & 2 & 350 & 2.0 \\
\hline 2001Q4 & 0.98 & 0.32 & 6.06 & UVES & 53130.960 & 24 & 120 & 80000 & $0.44 \times 8.00$ & 3 & 330 & 1.2 \\
\hline 2001Q4 & 0.98 & 0.32 & 6.06 & UVES & 53130.962 & 24 & 120 & 80000 & $0.44 \times 8.00$ & 13 & 420 & 0.9 \\
\hline 2001Q4 & 0.98 & 0.32 & 6.06 & UVES & 53130.965 & 24 & 120 & 80000 & $0.44 \times 8.00$ & 108 & - & - \\
\hline 2001Q4 & 0.98 & 0.32 & 6.06 & UVES & 53130.967 & 24 & 600 & 80000 & $0.44 \times 8.00$ & 108 & 330 & -0.0 \\
\hline 2001Q4 & 0.98 & 0.32 & 6.06 & UVES & 53130.975 & 24 & 7200 & 80000 & $0.44 \times 8.00$ & 217 & 330 & 0.1 \\
\hline 2001Q4 & 0.98 & 0.32 & 6.06 & UVES & 53131.066 & 24 & 2185 & 80000 & $0.44 \times 8.00$ & 13 & 420 & 1.0 \\
\hline 2001Q4 & 0.98 & 0.32 & 6.06 & UVES & 53131.952 & 26 & 1782 & 80000 & $0.44 \times 8.00$ & 110 & 330 & 0.1 \\
\hline 2001Q4 & 0.98 & 0.32 & 6.06 & UVES & 53131.991 & 26 & 6300 & 80000 & $0.44 \times 8.00$ & 216 & 400 & 0.8 \\
\hline 2001Q4 & 0.97 & 0.32 & 6.06 & UVES & 53132.065 & 26 & 2144 & 80000 & $0.44 \times 8.00$ & 13 & 330 & 1.2 \\
\hline $2003 \mathrm{~K} 4$ & 2.59 & 2.35 & 9.14 & UVES & 53132.343 & 27 & 4380 & 60000 & $0.60 \times 8.00$ & 0 & 200 & 0.1 \\
\hline 2003K4 & 1.19 & 1.53 & 5.78 & UVES & 53328.347 & 32 & 1500 & 80000 & $0.44 \times 10.00$ & 0 & 320 & 1.7 \\
\hline 2003K4 & 1.20 & 1.51 & 5.80 & UVES & 53329.344 & 32 & 1500 & 80000 & $0.44 \times 10.00$ & 0 & 330 & 1.7 \\
\hline Tempel1 & 1.55 & 0.75 & 10.71 & HIRES & 53520.363 & 33 & 1200 & 47000 & $0.86 \times 7.00$ & 0 & 350 & 1.2 \\
\hline Tempel1 & 1.55 & 0.75 & 10.71 & HIRES & 53520.378 & 33 & 1200 & 47000 & $0.86 \times 7.00$ & 0 & 380 & 1.0 \\
\hline Tempel1 & 1.55 & 0.75 & 10.71 & HIRES & 53520.392 & 33 & 1200 & 47000 & $0.86 \times 7.00$ & 0 & 380 & 1.1 \\
\hline Tempel1 & 1.54 & 0.76 & 10.69 & UVES & 53523.016 & 34 & 5400 & 80000 & $0.44 \times 10.00$ & 0 & 320 & 1.2 \\
\hline Tempel1 & 1.54 & 0.76 & 10.69 & UVES & 53523.083 & 34 & 5400 & 80000 & $0.44 \times 10.00$ & 0 & 290 & 1.2 \\
\hline Tempel1 & 1.53 & 0.78 & 10.65 & UVES & 53528.025 & 34 & 5400 & 80000 & $0.44 \times 10.00$ & 0 & 310 & 1.2 \\
\hline Tempel1 & 1.53 & 0.78 & 10.65 & UVES & 53528.091 & 34 & 5400 & 80000 & $0.44 \times 10.00$ & 0 & 290 & 1.2 \\
\hline Tempel1 & 1.53 & 0.78 & 10.63 & UVES & 53529.033 & 34 & 5400 & 80000 & $0.44 \times 10.00$ & 0 & 320 & 1.2 \\
\hline Tempel1 & 1.53 & 0.78 & 10.63 & UVES & 53529.102 & 34 & 5400 & 80000 & $0.44 \times 10.00$ & 0 & 310 & 1.2 \\
\hline Tempel1 & 1.51 & 0.89 & 10.36 & UVES & 53553.955 & 35 & 7200 & 80000 & $0.44 \times 10.00$ & 1 & 300 & 0.8 \\
\hline Tempel1 & 1.51 & 0.89 & 10.36 & UVES & 53554.041 & 35 & 7200 & 80000 & $0.44 \times 10.00$ & 1 & 280 & 0.1 \\
\hline Tempel1 & 1.51 & 0.89 & 10.34 & UVES & 53554.954 & 35 & 7200 & 80000 & $0.44 \times 10.00$ & 1 & - & - \\
\hline Tempel1 & 1.51 & 0.89 & 10.34 & UVES & 535 & 35 & 7200 & 80000 & $0.44 \times 10.00$ & 1 & 210 & -0.4 \\
\hline Tempel1 & 1.51 & 0.89 & 10.34 & HIRES & 53555.238 & 36 & 720 & 47000 & $0.86 \times 7.00$ & 0 & 420 & 3.2 \\
\hline Tempel1 & 1.51 & 0.89 & 10.34 & HIRES & 53555.250 & 36 & 600 & 47000 & $0.86 \times 7.00$ & 0 & 310 & 3.2 \\
\hline Tempel1 & 1.51 & 0.89 & 10.34 & HIRES & 53555.258 & 36 & 600 & 47000 & $0.86 \times 7.00$ & 0 & 210 & 1.8 \\
\hline Tempel1 & 1.51 & 0.89 & 10.34 & HIRES & 53555.267 & 36 & 900 & 47000 & $0.86 \times 7.00$ & 0 & 200 & 1.7 \\
\hline Tempel1 & 1.51 & 0.89 & 10.34 & HIRES & 53555.278 & 36 & 900 & 47000 & $0.86 \times 7.00$ & 0 & 260 & 2.2 \\
\hline Tempel1 & 1.51 & 0.89 & 10.34 & HIRES & 53555.289 & 36 & 900 & 47000 & $0.86 \times 7.00$ & 0 & 240 & 1.8 \\
\hline Tempel1 & 1.51 & 0.89 & 10.34 & HIRES & 53555.300 & 36 & 900 & 47000 & $0.86 \times 7.00$ & 0 & 210 & 1.2 \\
\hline Tempel1 & 1.51 & 0.89 & 10.34 & HIRES & 53555.311 & 36 & 900 & 47000 & $0.86 \times 7.00$ & 0 & 210 & 1.2 \\
\hline Tempel1 & 1.51 & 0.89 & 10.34 & HIRES & 53555.322 & 36 & 900 & 47000 & $0.86 \times 7.00$ & 0 & 230 & 1.2 \\
\hline Tempel1 & 1.51 & 0.89 & 10.34 & HIRES & 53555.333 & 36 & 900 & 47000 & $0.86 \times 7.00$ & 0 & 230 & 1.2 \\
\hline Tempel1 & 1.51 & 0.89 & 10.34 & HIRES & 53555.344 & 36 & 900 & 47000 & $0.86 \times 7.00$ & 0 & - & - \\
\hline Tempel1 & 1.51 & 0.89 & 10.34 & HIRES & 53555.355 & 36 & 900 & 47000 & $0.86 \times 7.00$ & 0 & 230 & 1.2 \\
\hline Tempel1 & 1.51 & 0.89 & 10.34 & HIRES & 53555.372 & 36 & 1800 & 47000 & $0.86 \times 7.00$ & 0 & 250 & 1.0 \\
\hline Tempel1 & 1.51 & 0.89 & 10.34 & HIRES & 53555.393 & 36 & 1800 & 47000 & $0.86 \times 7.00$ & 0 & 250 & 1.2 \\
\hline Tempel1 & 1.51 & 0.90 & 10.33 & UVES & 53555.955 & 37 & 7200 & 80000 & $0.44 \times 10.00$ & 1 & 260 & -0.3 \\
\hline Tempel1 & 1.51 & 0.90 & 10.33 & UVES & 53556.043 & 37 & 7800 & 80000 & $0.44 \times 10.00$ & 2 & 260 & 0.9 \\
\hline Tempel1 & 1.51 & 0.90 & 10.41 & UVES & 53557.007 & 37 & 9600 & 80000 & $0.44 \times 10.00$ & 1 & 270 & 1.1 \\
\hline Tempel1 & 1.51 & 0.90 & 10.41 & UVES & 53557.121 & 37 & 4800 & 80000 & $0.44 \times 10.00$ & 0 & 280 & -0.0 \\
\hline Tempel1 & 1.51 & 0.91 & 10.40 & UVES & 53557.955 & 37 & 7500 & 80000 & $0.44 \times 10.00$ & 0 & 240 & 0.1 \\
\hline Tempel1 & 1.51 & 0.91 & 10.40 & UVES & 53558.044 & 37 & 7500 & 80000 & $0.44 \times 10.00$ & 0 & - & - \\
\hline Tempel1 & 1.51 & 0.91 & 10.39 & UVES & 53558.952 & 37 & 7500 & 80000 & $0.44 \times 10.00$ & 0 & 280 & 0.9 \\
\hline Tempel1 & 1.51 & 0.92 & 10.39 & UVES & 53559.041 & 37 & 7500 & 80000 & $0.44 \times 10.00$ & 0 & - & - \\
\hline
\end{tabular}


Table 8. continued.

\begin{tabular}{|c|c|c|c|c|c|c|c|c|c|c|c|c|}
\hline$\overline{\text { Comet }}$ & $\begin{array}{l}r \\
(\mathrm{AU})\end{array}$ & $\begin{array}{l}\Delta \\
(\mathrm{AU})\end{array}$ & $\overline{m_{r}}$ & $\overline{\text { Spectro }}$ & $\overline{\mathrm{MJD}}$ & $\overline{\text { Run }}$ & $\begin{array}{r}\operatorname{Exp} \\
(\mathrm{s})\end{array}$ & $\bar{R}$ & $\begin{array}{r}\text { Slit } \\
\left({ }^{\prime \prime} x^{\prime \prime}\right)\end{array}$ & $\begin{array}{r}\text { Offset } \\
\left({ }^{\prime \prime}\right)\end{array}$ & $\begin{array}{c}T \\
(\mathrm{~K})\end{array}$ & $\begin{array}{c}5+\log Q \\
\left(\mathrm{~s}^{-1}\right)\end{array}$ \\
\hline Tempel1 & 1.51 & 0.92 & 10.38 & UVES & 53559.954 & 37 & 7500 & 80000 & $0.44 \times 10.00$ & 0 & 280 & 1.2 \\
\hline Tempel1 & 1.51 & 0.92 & 10.48 & UVES & 53560.044 & 37 & 7500 & 80000 & $0.44 \times 10.00$ & 0 & 300 & 0.8 \\
\hline Tempel1 & 1.51 & 0.93 & 10.46 & UVES & 53560.952 & 37 & 7800 & 80000 & $0.44 \times 10.00$ & 0 & 290 & 1.2 \\
\hline Tempel1 & 1.51 & 0.93 & 10.46 & UVES & 53561.045 & 37 & 7800 & 80000 & $0.44 \times 10.00$ & 0 & - & - \\
\hline Tempel1 & 1.51 & 0.93 & 10.45 & UVES & 53561.953 & 37 & 7200 & 80000 & $0.44 \times 10.00$ & 0 & 280 & 1.2 \\
\hline Tempel1 & 1.51 & 0.93 & 10.55 & UVES & 53562.041 & 37 & 7200 & 80000 & $0.44 \times 10.00$ & 0 & 270 & 0.9 \\
\hline Tempel1 & 1.51 & 0.94 & 10.54 & UVES & 53562.955 & 37 & 7200 & 80000 & $0.44 \times 10.00$ & 0 & 280 & 1.0 \\
\hline Tempel1 & 1.51 & 0.94 & 10.54 & UVES & 53563.041 & 37 & 7200 & 80000 & $0.44 \times 10.00$ & 0 & 230 & 0.1 \\
\hline SW3-B & 1.05 & 0.10 & 11.02 & 2DCoudé & 53857.209 & 38 & 300 & 60000 & $1.20 \times 8.20$ & 0 & 320 & 2.5 \\
\hline SW3-B & 1.07 & 0.12 & 10.65 & 2DCoudé & 53860.322 & 40 & 1800 & 60000 & $1.20 \times 8.20$ & 0 & 280 & 2.5 \\
\hline SW3-B & 0.95 & 0.15 & 11.60 & UVES & 53882.367 & 41 & 4800 & 80000 & $0.60 \times 10.00$ & 0 & 390 & 1.9 \\
\hline SW3-B & 0.95 & 0.16 & 11.50 & UVES & 53883.353 & 41 & 3609 & 80000 & $0.60 \times 10.00$ & 2 & 350 & 1.2 \\
\hline SW3-B & 0.95 & 0.16 & 11.50 & UVES & 53883.398 & 41 & 2400 & 80000 & $0.60 \times 10.00$ & 2 & 360 & 1.2 \\
\hline SW3-C & 1.06 & 0.10 & 11.07 & 2DCoudé & 53858.190 & 39 & 1800 & 60000 & $1.20 \times 8.20$ & 0 & 270 & 2.5 \\
\hline SW3-C & 1.07 & 0.11 & 10.95 & 2DCoudé & 53859.212 & 39 & 1800 & 60000 & $1.20 \times 8.20$ & 0 & 340 & 1.9 \\
\hline SW3-C & 1.07 & 0.12 & 10.79 & 2DCoudé & 53860.183 & 39 & 1800 & 60000 & $1.20 \times 8.20$ & 0 & 380 & 2.5 \\
\hline SW3-C & 0.94 & 0.25 & 11.39 & UVES & 53898.369 & 42 & 4800 & 80000 & $1.00 \times 10.00$ & 0 & 340 & 1.2 \\
\hline SW3-C & 1.13 & 0.50 & 14.52 & UVES & 53937.345 & 43 & 5000 & 80000 & $0.60 \times 10.00$ & 0 & 370 & 1.7 \\
\hline SWAN & 1.00 & 1.02 & 5.25 & 2DCoudé & 54039.052 & 44 & 1800 & 60000 & $1.20 \times 8.20$ & 0 & 350 & 1.9 \\
\hline SWAN & 1.00 & 1.02 & 5.25 & 2DCoudé & 54039.074 & 44 & 1800 & 60000 & $1.20 \times 8.20$ & 0 & 350 & 1.8 \\
\hline Holmes & 2.44 & 1.63 & 1.43 & 2DCoudé & 54398.400 & 45 & 60 & 60000 & $1.20 \times 8.20$ & 20 & 160 & 3.2 \\
\hline Holmes & 2.44 & 1.63 & 1.43 & 2DCoudé & 54398.406 & 45 & 600 & 60000 & $1.20 \times 8.20$ & 20 & 140 & 2.5 \\
\hline Holmes & 2.45 & 1.63 & 1.43 & 2DCoudé & 54401.290 & 45 & 300 & 60000 & $1.20 \times 8.20$ & 20 & 180 & 0.9 \\
\hline Holmes & 2.45 & 1.63 & 1.43 & 2DCoudé & 54401.300 & 45 & 600 & 60000 & $1.20 \times 8.20$ & 20 & 170 & 1.0 \\
\hline Holmes & 2.46 & 1.63 & 1.43 & 2DCoudé & 54402.362 & 45 & 1200 & 60000 & $1.20 \times 8.20$ & 20 & 200 & 0.9 \\
\hline Holmes & 2.46 & 1.63 & 1.43 & HIRES & 54402.573 & 46 & 730 & 47000 & $0.86 \times 7.00$ & 0 & 160 & 2.1 \\
\hline Holmes & 2.46 & 1.62 & 1.45 & 2DCoudé & 54403.362 & 47 & 1200 & 60000 & $1.20 \times 8.20$ & 20 & 160 & 1.1 \\
\hline Holmes & 2.46 & 1.62 & 1.45 & 2DCoudé & 54404.383 & 47 & 1200 & 60000 & $1.20 \times 8.20$ & 20 & 170 & 1.0 \\
\hline Holmes & 2.54 & 1.64 & 1.92 & 2DCoudé & 54422.366 & 48 & 1800 & 60000 & $1.20 \times 8.20$ & 20 & 200 & 1.2 \\
\hline Holmes & 2.54 & 1.64 & 1.92 & 2DCoudé & 54422.388 & 48 & 1800 & 60000 & $1.20 \times 8.20$ & 20 & 180 & 1.0 \\
\hline Holmes & 2.54 & 1.64 & 1.92 & 2DCoudé & 54422.410 & 48 & 1800 & 60000 & $1.20 \times 8.20$ & 20 & 200 & 1.9 \\
\hline Holmes & 2.54 & 1.64 & 1.92 & 2DCoudé & 54422.432 & 48 & 1800 & 60000 & $1.20 \times 8.20$ & 20 & 210 & 0.9 \\
\hline Holmes & 2.54 & 1.64 & 1.92 & 2DCoudé & 54423.253 & 48 & 1800 & 60000 & $1.20 \times 8.20$ & 20 & 210 & 0.8 \\
\hline Holmes & 2.54 & 1.64 & 1.92 & 2DCoudé & 54423.275 & 48 & 1800 & 60000 & $1.20 \times 8.20$ & 20 & 200 & 2.0 \\
\hline Holmes & 2.54 & 1.64 & 1.92 & 2DCoudé & 54423.297 & 48 & 1800 & 60000 & $1.20 \times 8.20$ & 20 & 200 & 0.9 \\
\hline Tuttle & 1.04 & 0.36 & 8.43 & UVES & 54481.021 & 49 & 3600 & 80000 & $0.44 \times 10.00$ & 0 & 400 & 1.7 \\
\hline Tuttle & 1.04 & 0.36 & 8.42 & UVES & 54481.071 & 49 & 4800 & 80000 & $0.44 \times 10.00$ & 0 & 370 & 1.7 \\
\hline Tuttle & 1.03 & 0.52 & 8.01 & UVES & 54493.018 & 49 & 3900 & 80000 & $0.44 \times 10.00$ & 0 & 310 & 1.8 \\
\hline Tuttle & 1.03 & 0.52 & 8.00 & UVES & 54493.071 & 49 & 3900 & 80000 & $0.44 \times 10.00$ & 0 & 290 & 1.9 \\
\hline Tuttle & 1.03 & 0.62 & 7.73 & UVES & 54500.017 & 49 & 3900 & 80000 & $0.44 \times 10.00$ & 0 & 310 & 1.9 \\
\hline Tuttle & 1.03 & 0.62 & 7.73 & UVES & 54500.070 & 49 & 3900 & 80000 & $0.44 \times 10.00$ & 0 & 300 & 1.8 \\
\hline Lulin & 1.33 & 0.53 & 6.97 & 2DCoudé & 54876.328 & 50 & 1800 & 60000 & $1.20 \times 8.20$ & 0 & 280 & 2.5 \\
\hline Lulin & 1.33 & 0.53 & 6.97 & 2DCoudé & 54876.351 & 50 & 1800 & 60000 & $1.20 \times 8.20$ & 0 & 390 & 3.1 \\
\hline Lulin & 1.33 & 0.53 & 6.97 & 2DCoudé & 54876.373 & 50 & 1800 & 60000 & $1.20 \times 8.20$ & 0 & 270 & 2.6 \\
\hline Lulin & 1.33 & 0.53 & 6.97 & 2DCoudé & 54876.395 & 50 & 1800 & 60000 & $1.20 \times 8.20$ & 0 & 300 & 2.8 \\
\hline Lulin & 1.33 & 0.53 & 6.97 & 2DCoudé & 54876.418 & 50 & 1800 & 60000 & $1.20 \times 8.20$ & 0 & 260 & 2.5 \\
\hline Lulin & 1.33 & 0.53 & 6.97 & 2DCoudé & 54876.440 & 50 & 1800 & 60000 & $1.20 \times 8.20$ & 0 & 280 & 2.5 \\
\hline Lulin & 1.33 & 0.53 & 6.97 & 2DCoudé & 54876.463 & 50 & 1800 & 60000 & $1.20 \times 8.20$ & 0 & 290 & 2.7 \\
\hline Lulin & 1.33 & 0.53 & 6.97 & 2DCoudé & 54876.486 & 50 & 1800 & 60000 & $1.20 \times 8.20$ & 0 & 260 & 2.5 \\
\hline Lulin & 1.33 & 0.53 & 6.97 & 2DCoudé & 54876.508 & 50 & 1800 & 60000 & $1.20 \times 8.20$ & 0 & 350 & 2.5 \\
\hline Lulin & 1.33 & 0.53 & 6.87 & 2DCoudé & 54877.351 & 50 & 1800 & 60000 & $1.20 \times 8.20$ & 4 & 270 & 2.5 \\
\hline Lulin & 1.33 & 0.53 & 6.87 & 2DCoudé & 54877.374 & 50 & 1800 & 60000 & $1.20 \times 8.20$ & 4 & 280 & 2.6 \\
\hline Lulin & 1.33 & 0.53 & 6.87 & 2DCoudé & 54877.397 & 50 & 1800 & 60000 & $1.20 \times 8.20$ & 4 & 270 & 2.5 \\
\hline Lulin & 1.33 & 0.53 & 6.87 & 2DCoudé & 54877.419 & 50 & 1800 & 60000 & $1.20 \times 8.20$ & 4 & 270 & 2.5 \\
\hline Lulin & 1.33 & 0.53 & 6.87 & 2DCoudé & 54877.441 & 50 & 1800 & 60000 & $1.20 \times 8.20$ & 4 & 280 & 2.5 \\
\hline Lulin & 1.33 & 0.53 & 6.87 & 2DCoudé & 54877.463 & 50 & 1800 & 60000 & $1.20 \times 8.20$ & 4 & 260 & 2.6 \\
\hline Lulin & 1.33 & 0.53 & 6.87 & 2DCoudé & 54877.485 & 50 & 1800 & 60000 & $1.20 \times 8.20$ & 4 & 270 & 2.7 \\
\hline Lulin & 1.33 & 0.53 & 6.87 & 2DCoudé & 54877.507 & 50 & 1800 & 60000 & $1.20 \times 8.20$ & 4 & 260 & 2.5 \\
\hline
\end{tabular}




\section{Appendix A: Estimating the isotopic ratios}

Because of uncertainties in the models and systematic errors in the observations, the parameters $\alpha$ and $\beta$ (Eq. (5)) cannot be estimated directly. Additional parameters are required to deal with the exact central wavelength of the lines, and the exact level of a possible residual background $C_{k}(\lambda)$.

The spectra are divided into small domains surrounding the central wavelength $\lambda_{i}$ of the most intense, unblended $R$ lines of ${ }^{13} \mathrm{C}^{14} \mathrm{~N}$ and ${ }^{12} \mathrm{C}^{15} \mathrm{~N}$, i.e., regions where $\alpha S_{k, 2}(\lambda)$ or $\beta S_{k, 3}(\lambda) \gg S_{k, 1}(\lambda)$. This is necessary because the accuracy of the ${ }^{12} \mathrm{C}^{14} \mathrm{~N}$ model is not perfect, especially in the wings of intense lines. Lines of other molecules must also be avoided, e.g., an unidentified feature at $\lambda \sim 3867.92 \AA$ precludes the use of the $\mathrm{R} 10$ line of ${ }^{12} \mathrm{C}^{15} \mathrm{~N}$ close to the nucleus (see Sect. 7).

The line profile of the strongest lines can be fitted by a Gaussian, or their intensity can be estimated by direct integration, providing sets of $\alpha$ and $\beta$ which can then be averaged.

However, in order to reduce the number of free parameters, we used a different procedure. Instead of fitting separately $O(\lambda)-C_{i}$ over the intervals $\left[\lambda_{i}-\Delta \lambda / 2, \lambda_{i}+\Delta \lambda / 2\right]$, we superimpose the profiles by shifting the line centers to $\lambda=0$, optimally coadd them, and fit the resulting profile over the resulting domain $[-\Delta \lambda / 2, \Delta \lambda / 2]$ (see Fig. A.1). Hence, dropping the subscript $k$, we write, for the ${ }^{13} \mathrm{C}^{14} \mathrm{~N}$ lines,

$\sum_{i} w_{i}\left[O\left(\lambda+\lambda_{i}\right)-C_{i}\right] \equiv \sum_{i} w_{i} O\left(\lambda+\lambda_{i}\right)-C=\alpha \sum_{i} w_{i} S_{2}\left(\lambda+\lambda_{i}\right)(\mathrm{A} .1)$

and the corresponding formula with $S_{3}$ and $\beta$ for the ${ }^{12} \mathrm{C}^{15} \mathrm{~N}$ lines. The $C_{i} \mathrm{~s}$ merge into a single free parameter $\sum_{i} w_{i} C_{i} \equiv C$. The weight factor $w_{i}$ is estimated from the expected intensity $f\left(\lambda_{i}\right) I_{i}$ of line $i$ in the SN-normalized spectrum (Eqs. (2), (3)).

The width of the observed "coadded" profile $\sum_{i} w_{i} O\left(\lambda+\lambda_{i}\right)$ is found to be equal to that of the synthetic profile $\sum_{i} w_{i} S_{2}\left(\lambda+\lambda_{i}\right)$ (or $\sum_{i} w_{i} S_{3}\left(\lambda+\lambda_{i}\right)$ ) and it is symmetric about zero. This confirms that the identification of the ${ }^{13} \mathrm{C}^{14} \mathrm{~N}$ and ${ }^{12} \mathrm{C}^{15} \mathrm{~N}$ lines is correct, as well as the theoretical wavelengths adopted for them.

The analysis is done either by profile fitting around $\lambda=0$ or by direct integration. In the latter case we write

$$
\int_{-\Delta \lambda / 2}^{\Delta \lambda / 2}\left[\sum_{i} w_{i} O\left(\lambda+\lambda_{i}\right)-C\right] \delta \lambda=\alpha \int_{-\Delta \lambda / 2}^{\Delta \lambda / 2} \sum_{i} w_{i} S_{2}\left(\lambda+\lambda_{i}\right) \delta \lambda(\mathrm{A} .2)
$$

for the ${ }^{13} \mathrm{C}^{14} \mathrm{~N}$ lines, and an equivalent formula for the ${ }^{12} \mathrm{C}^{15} \mathrm{~N}$ lines.

The choice of the lines $i$ depends on the quality of the spectra and on particular circumstances, especially the heliocentric distance. Figures 3 and 4 shows that the width of the envelope of the $\mathrm{CN}$ band decreases at large $r$. The relative intensity of the lines of high quantum number drops rapidly. Many lines could be used efficiently for the coadded profiles of comets de Vico or X5 (Fig. A.1), up to 11 for ${ }^{12} \mathrm{C}^{15} \mathrm{~N}$ and 8 for ${ }^{13} \mathrm{C}^{14} \mathrm{~N}$. On the contrary, at large $r$, a few lines dominate overwhelmingly. As shown in Sect. 7, some blends may become less troublesome far from the nucleus.

$R$ lines of ${ }^{13} \mathrm{C}^{14} \mathrm{~N}$ and ${ }^{12} \mathrm{C}^{15} \mathrm{~N}$ with low quantum numbers are slightly blended and also - depending on the spectral resolution - with the corresponding $\mathrm{R}$ line of ${ }^{12} \mathrm{C}^{14} \mathrm{~N}$. An additional difficulty is the presence of faint lines of the $\mathrm{B}-\mathrm{X}(0-0)$ band of $\mathrm{CH}$. This region of the spectrum needs special care (e.g., some iterative procedure) and may have to be ignored for the lowest quality spectra.

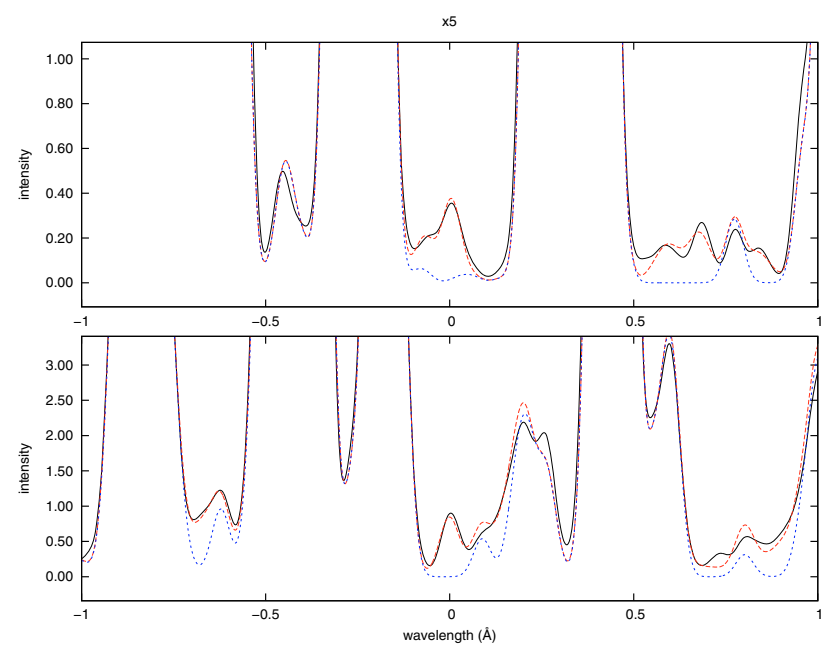

Fig. A.1. Coadded observed (solid line) and synthetic (with and without the rare isotopologues) spectra of comet C/2002 X5 (Kudo-Fujikawa). The upper panel is centered on ${ }^{13} \mathrm{C}^{14} \mathrm{~N}$ lines (in this instance, $\mathrm{R} 1-\mathrm{R} 6$, $\mathrm{R} 14$ and $\mathrm{R} 16)$, the lower one on ${ }^{12} \mathrm{C}^{15} \mathrm{~N}$ lines (R1-R5, R11-R13, $\mathrm{R} 15-\mathrm{R} 17)$. The large number of useable isotopic lines is allowed by the good quality of the spectra used in the combination. Intensity is in arbitrary units.

\section{Appendix B: Averages}

While weighted averages of measurements $x_{i}$ with errors $e_{i}$ $(i=1, \ldots, n)$ are easily defined as $m=\sum_{i} x_{i} e_{i}^{-2} / \sum_{i} e_{i}^{-2}$, estimating the resulting error on this value is less obvious. The global data set is far from homogeneous. Systematic errors affect the various data sets in different ways. The instrumentation and the circumstances are never identical. Two estimates of the standard error $S$ are sometimes used:

$S_{1}^{2}=\frac{\sum_{i}\left(x_{i}-m\right)^{2} e_{i}^{-2}}{(n-1) \sum_{i} e_{i}^{-2}}$

and

$S_{2}^{2}=1 / \sum_{i} e_{i}^{-2}$

They are not satisfying, particularly for a small data set. Equation (B.1) does not take properly into account the individual errors, except for the weighting factors, so that a few $x_{i}$ with large $e_{i}$ but grouped by chance around $m$ would give an unrealistically small $S_{1}$. On the other hand Eq. (B.2) does not take into account the inter-group variations which can be large in the case of systematic effects. The larger of $S_{1}$ and $S_{2}$ may be taken, but we choose a different approach by simulating the $x_{i}$ as the average of $n_{i}$ individual observations $y_{i, j}\left(j=1, \ldots, n_{i}\right)$ with a standard deviation $e_{i}$. The weighting is obtained by taking $n_{i}$ proportional to $e_{i}^{-2}$ i.e., the standard deviation is the same for each data set, $\sigma_{i}=\sigma$ and $n_{i}=\sigma^{2} e_{i}^{-2}$. It is then possible to combine several $x_{i}$ by merging their respective data sets. The mean value is the same as above and the standard deviation of the mean is given by

$e=\left(\frac{\sigma^{2} \sum_{i}\left(n_{i}-1\right)+\sum_{i} n_{i} x_{i}^{2}-\left(\sum_{i} x_{i} n_{i}\right)^{2} / \sum_{i} n_{i}}{\sum_{i} n_{i}\left(\sum_{i} n_{i}-1\right)}\right)^{1 / 2}$.

For large $\sigma$, this is equivalent to Eq. (B.2), i.e., the result is dominated by the internal dispersion of each data set. Choosing a reasonable value of $\sigma$ is thus critical. We adopt the value of $\sigma$ yielding the smallest realistic samples, $\min \left(n_{i}\right)=2$. This gives the largest, conservative estimates of the errors. 


\section{Appendix C: Spectra}

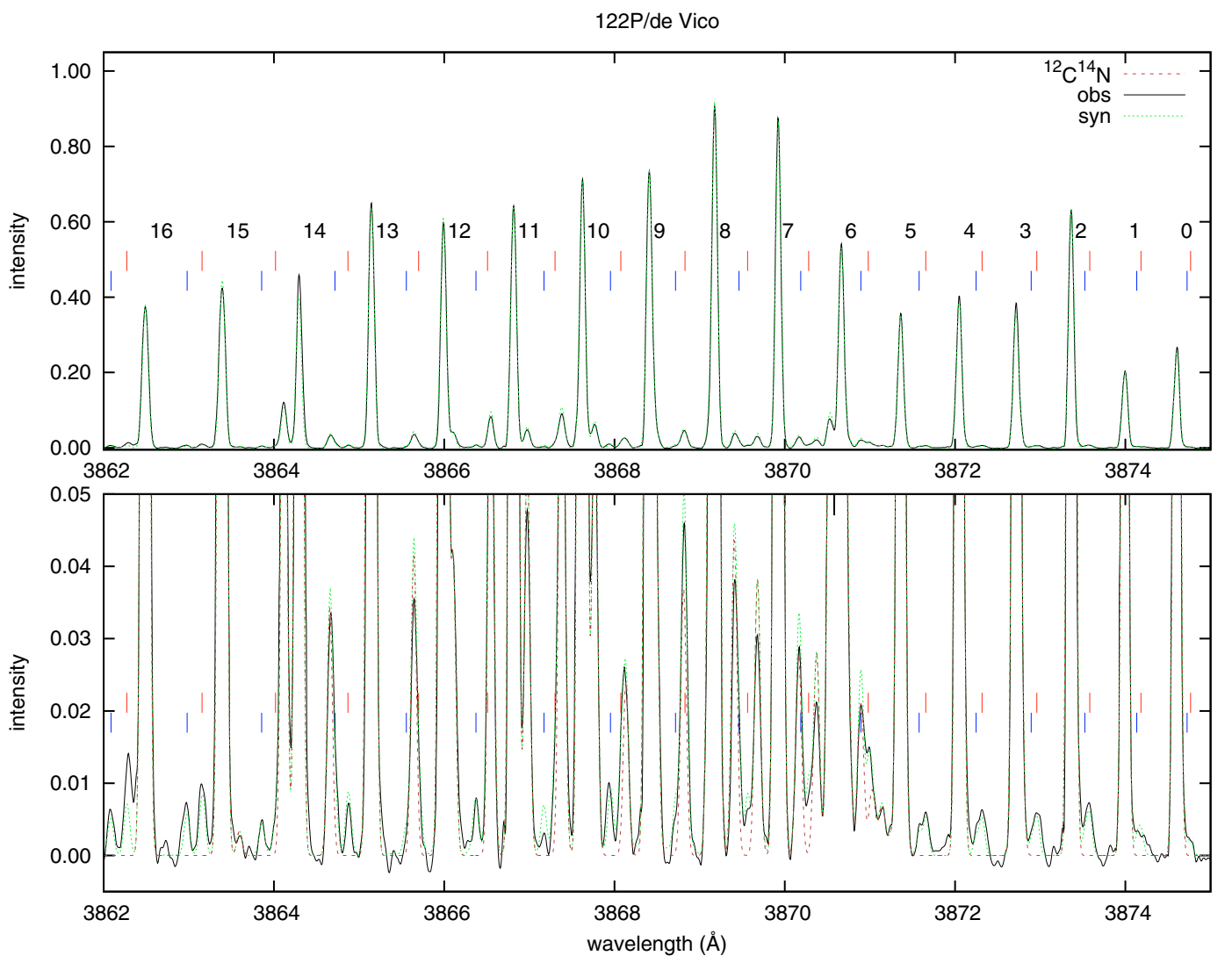

Fig. C.1. Observed (2DCoudé) and synthetic (dotted) spectra of comet 122P/de Vico. In this and the following graphs, the upper (red) ticks indicate the position of the major $\mathrm{R}$ lines of ${ }^{13} \mathrm{C}^{14} \mathrm{~N}$ the lower (blue) ticks indicate the position of the major $\mathrm{R}$ lines of ${ }^{12} \mathrm{C}^{15} \mathrm{~N}$. The corresponding quantum numbers are indicated in the upper panel midway between the strong ${ }^{12} \mathrm{C}^{14} \mathrm{~N}$ lines and the faint isotopic lines. The intensity scale is in relative units. 
J. Manfroid et al.: The $\mathrm{CN}$ isotopic ratios in comets, Online Material $p 7$

C/1996 B2 (Hyakutake)
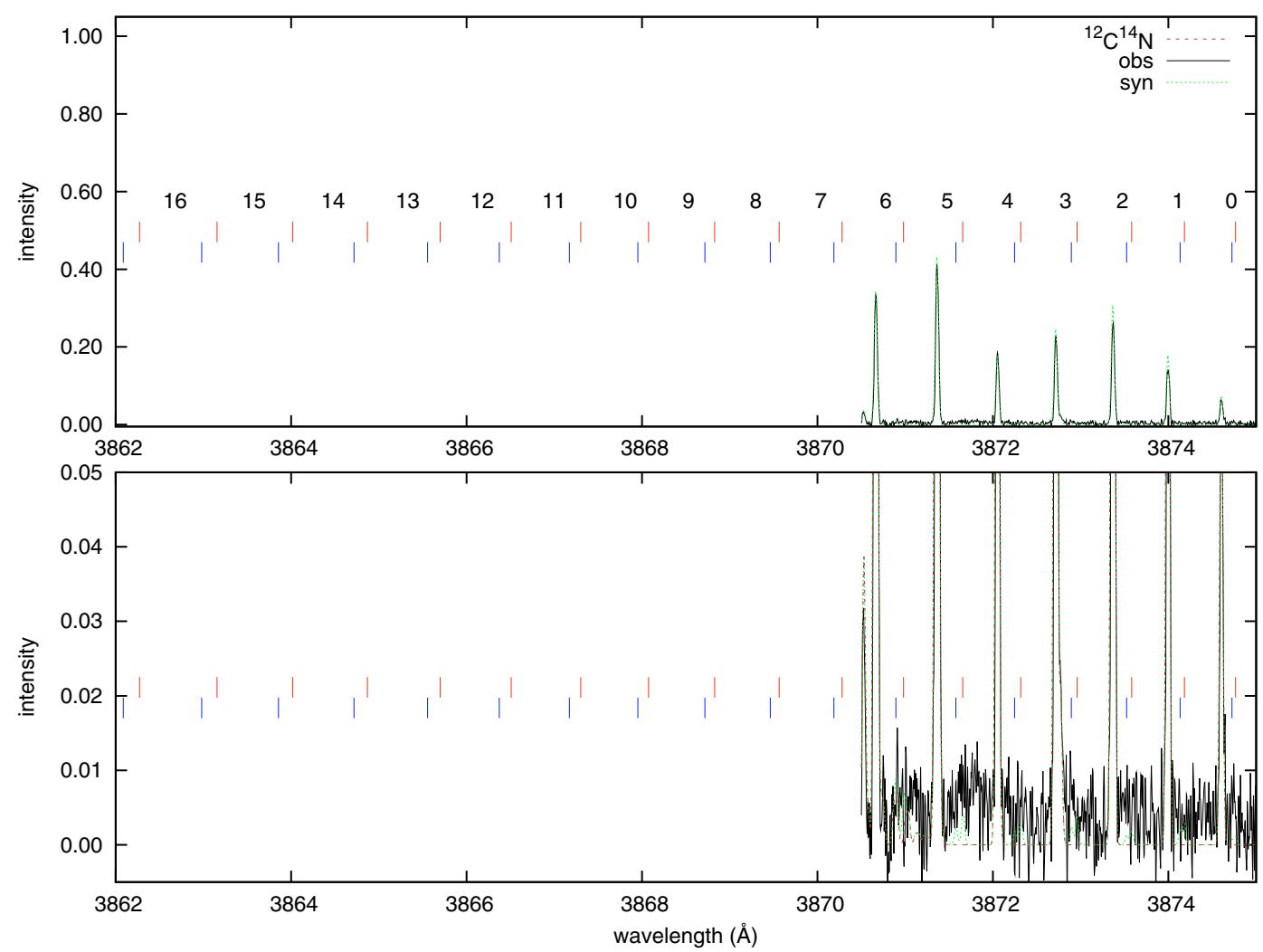

Fig. C.2. Observed (2DCoudé) and synthetic (dotted) spectra of comet C/1996 B2 (Hyakutake).

C/1996 B2 (Hyakutake)
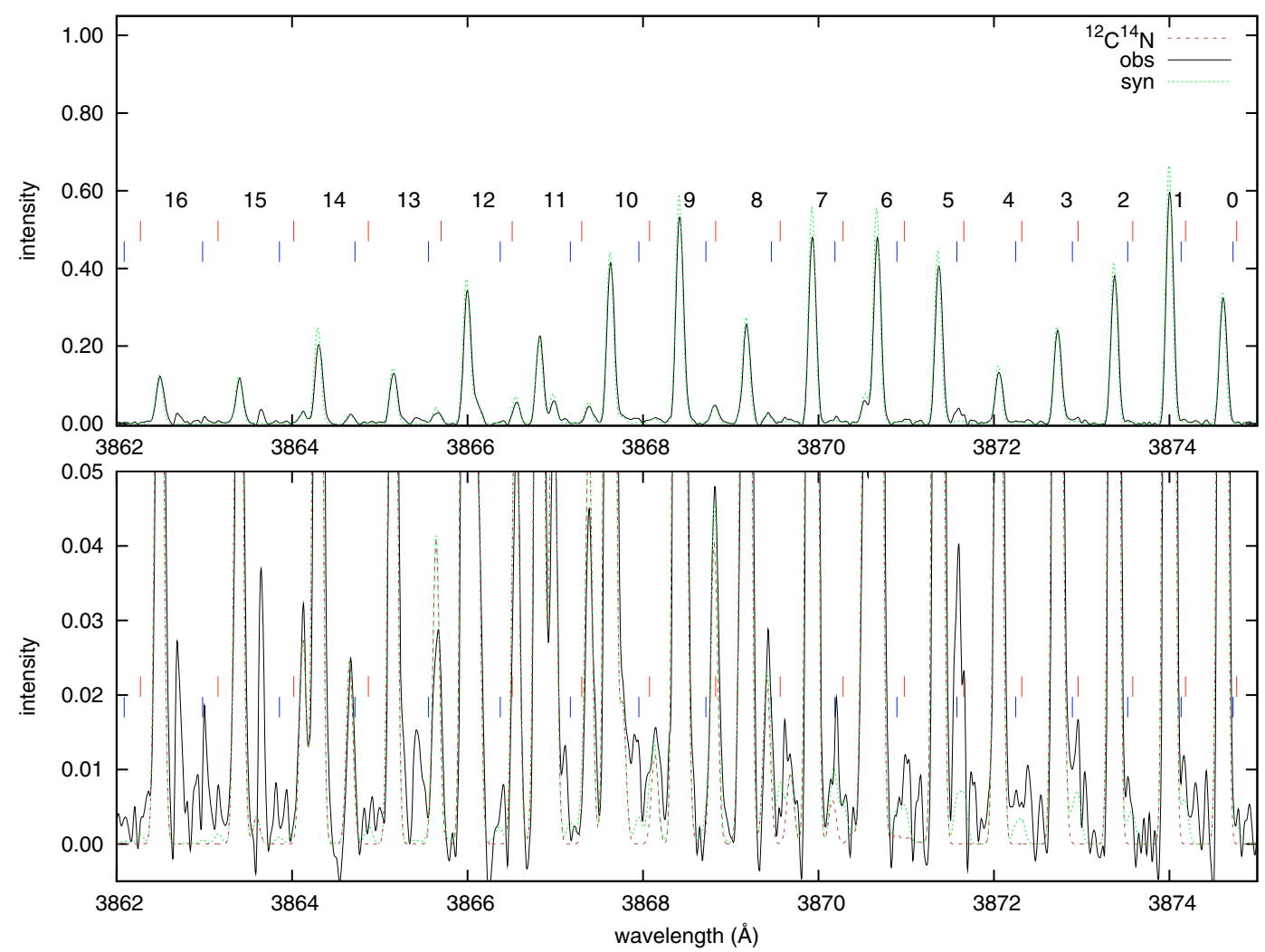

Fig. C.3. Observed (2DCoudé) and synthetic (dotted) spectra of comet C/1996 B2 (Hyakutake). 
J. Manfroid et al.: The $\mathrm{CN}$ isotopic ratios in comets, Online Material $p 8$

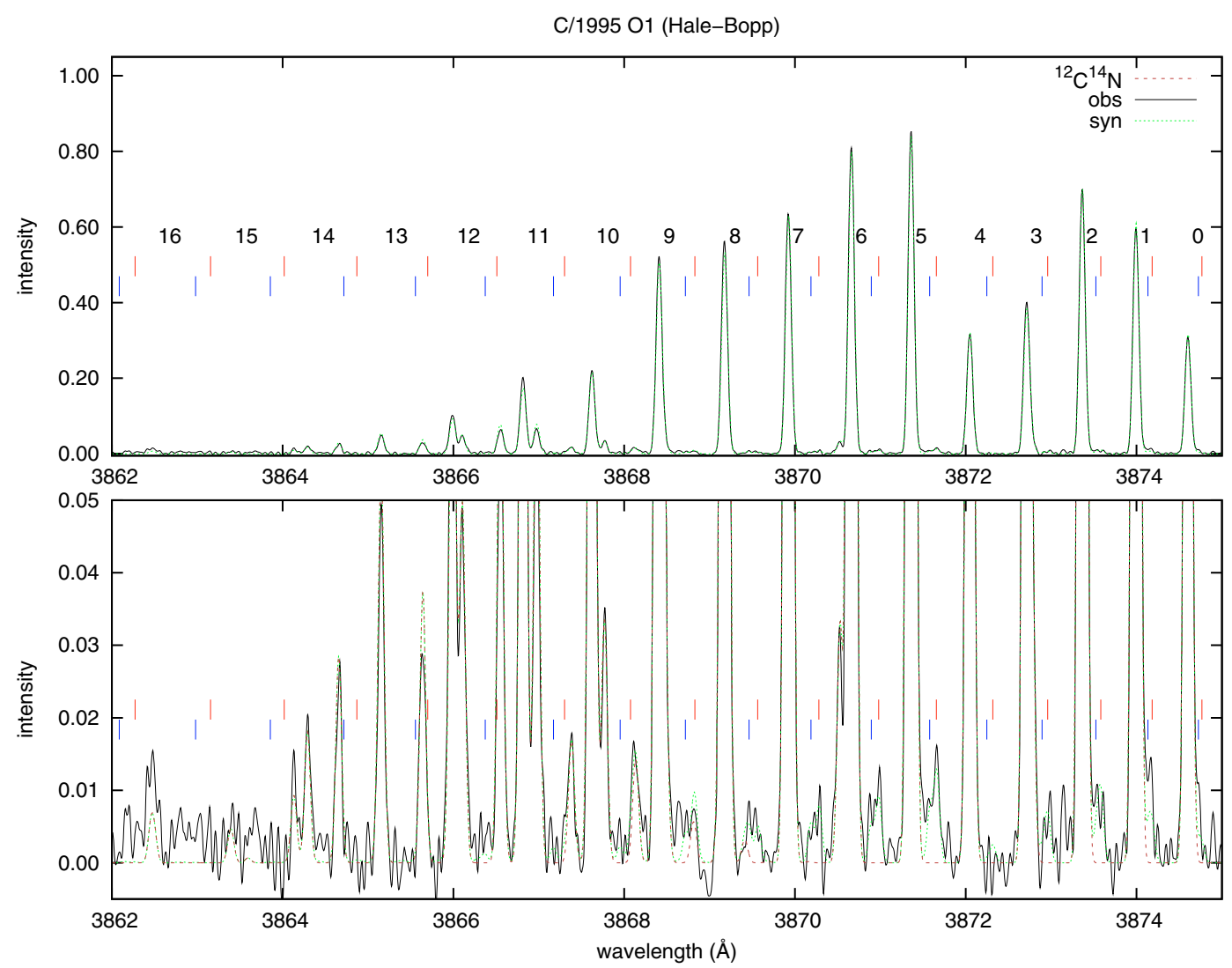

Fig. C.4. Observed (2DCoudé) and synthetic (dotted) spectra of comet C/1995 O1 (Hale-Bopp).
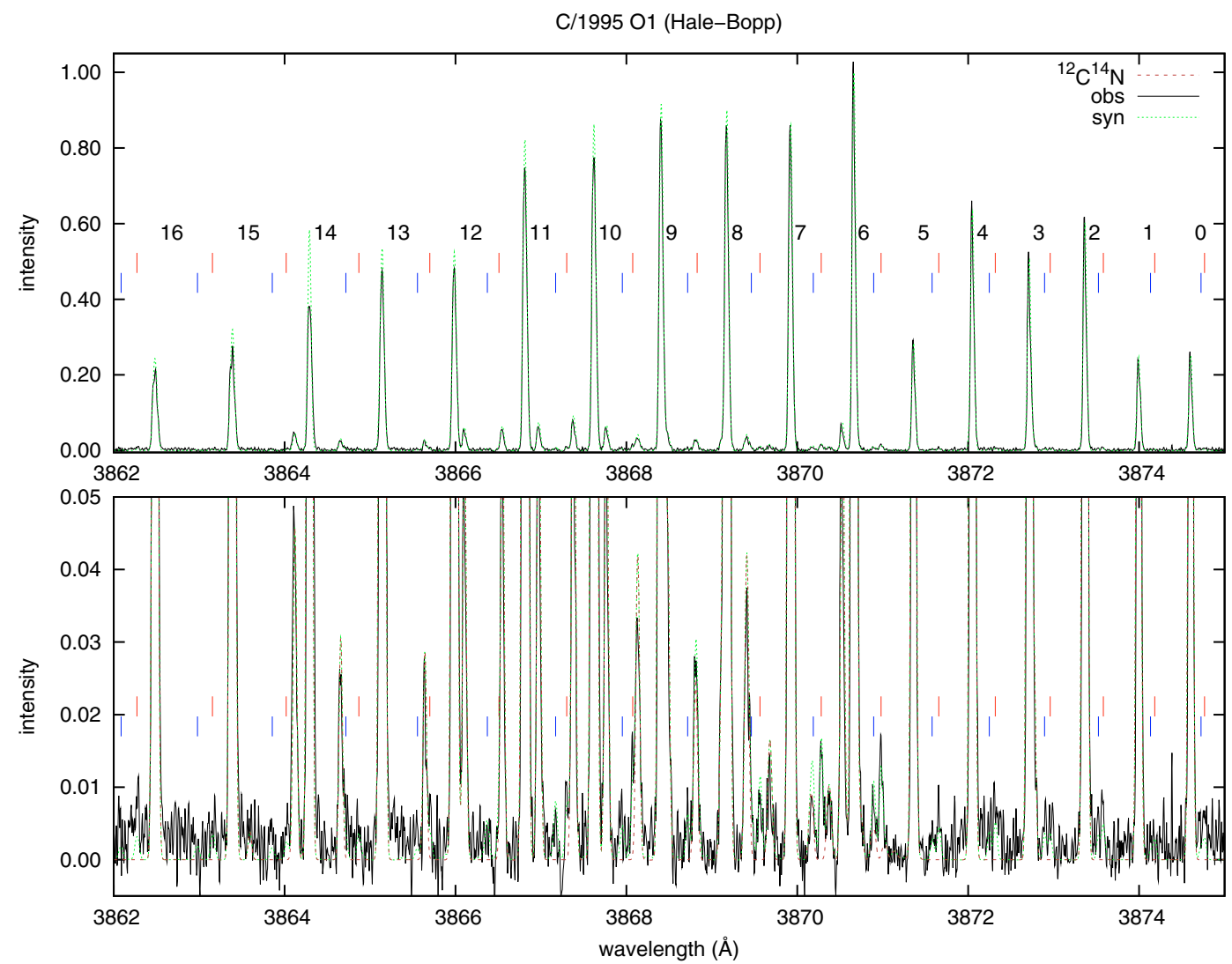

Fig. C.5. Observed (2DCoudé) and synthetic (dotted) spectra of comet C/1995 O1 (Hale-Bopp). 
J. Manfroid et al.: The $\mathrm{CN}$ isotopic ratios in comets, Online Material p 9
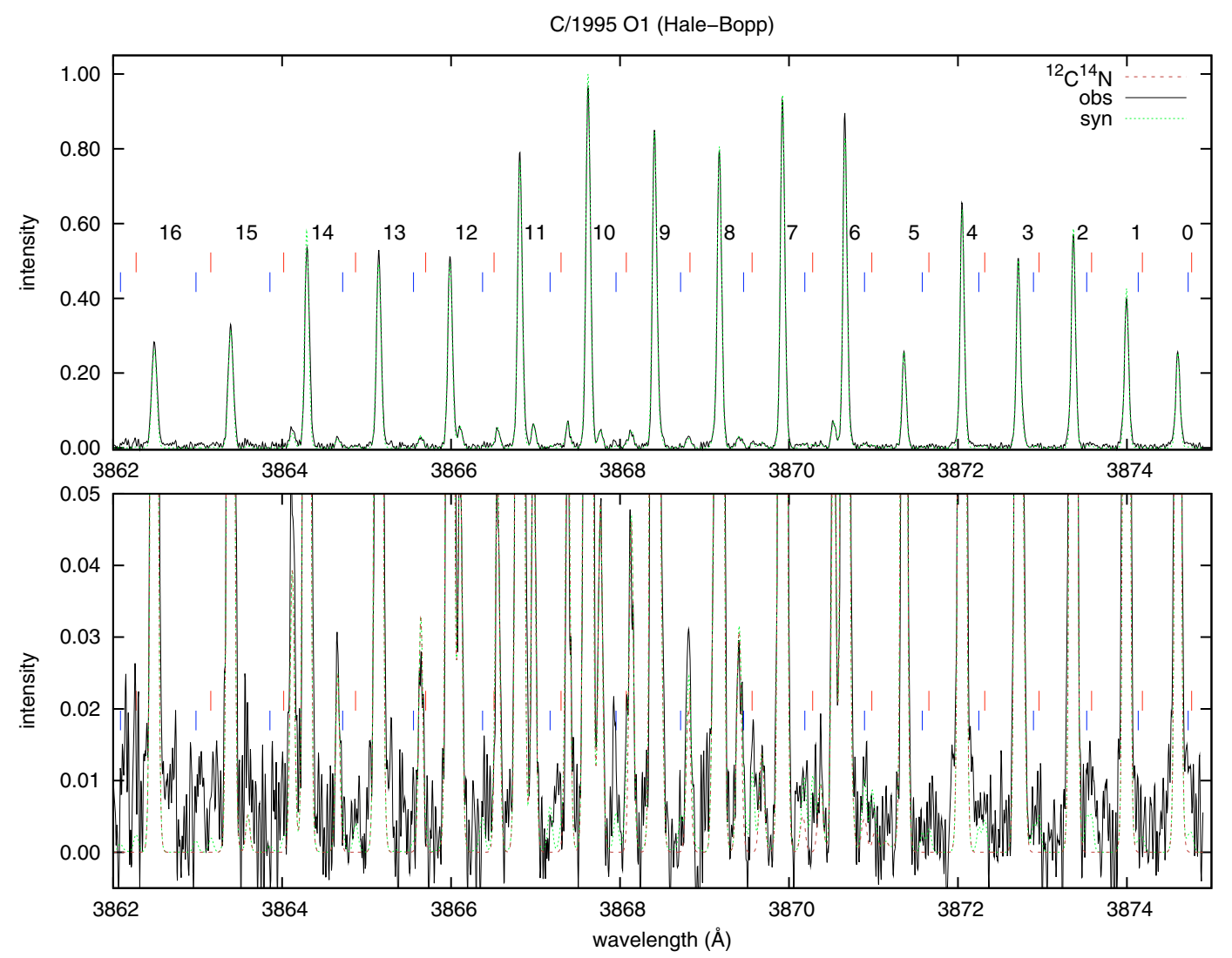

Fig. C.6. Observed (SOFIN) and synthetic (dotted) spectra of comet C/1995 O1 (Hale-Bopp).

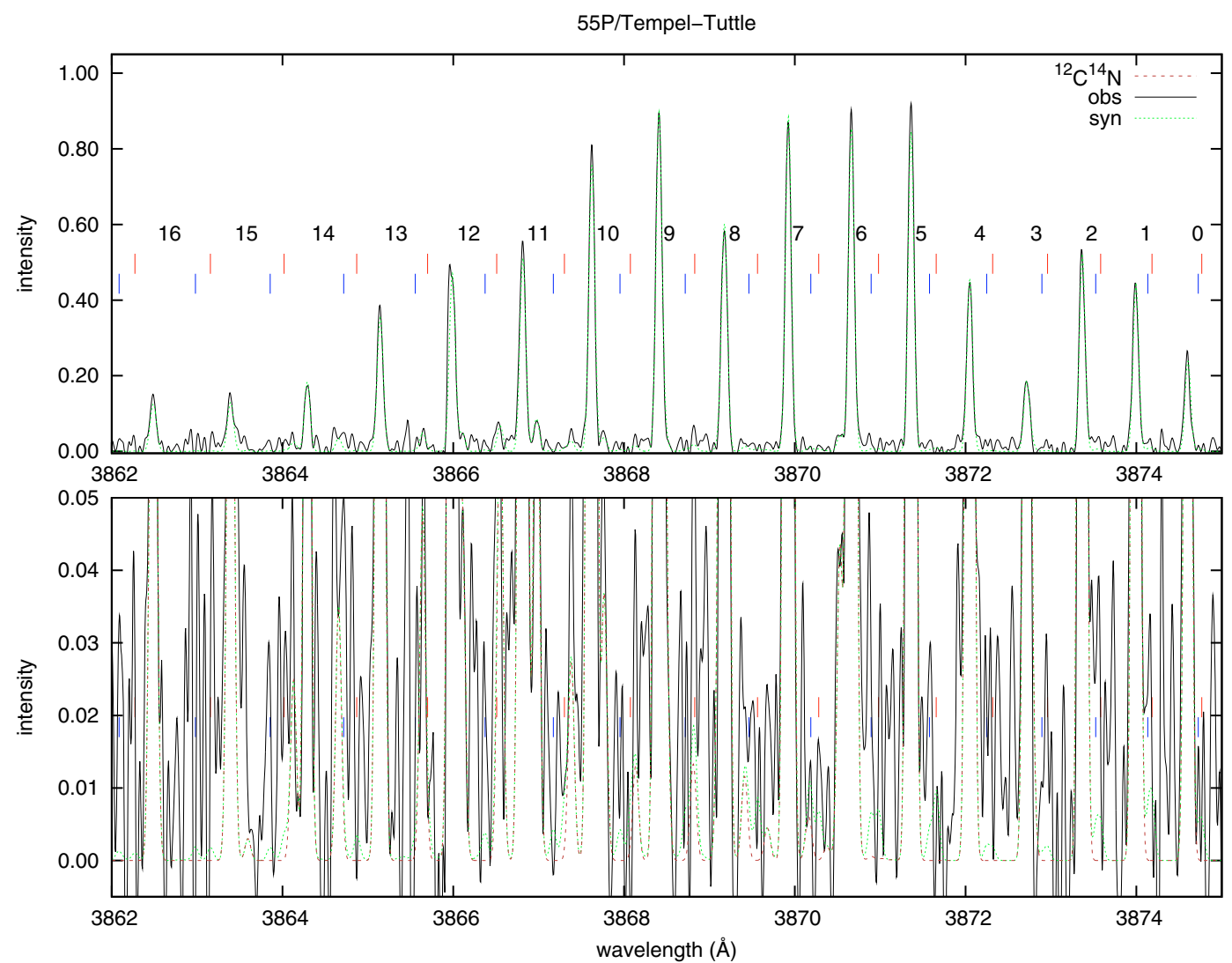

Fig. C.7. Observed (2DCoudé) and synthetic (dotted) spectra of comet 55P/Tempel-Tuttle. 
J. Manfroid et al.: The $\mathrm{CN}$ isotopic ratios in comets, Online Material p 10

C/1999 H1 (Lee)
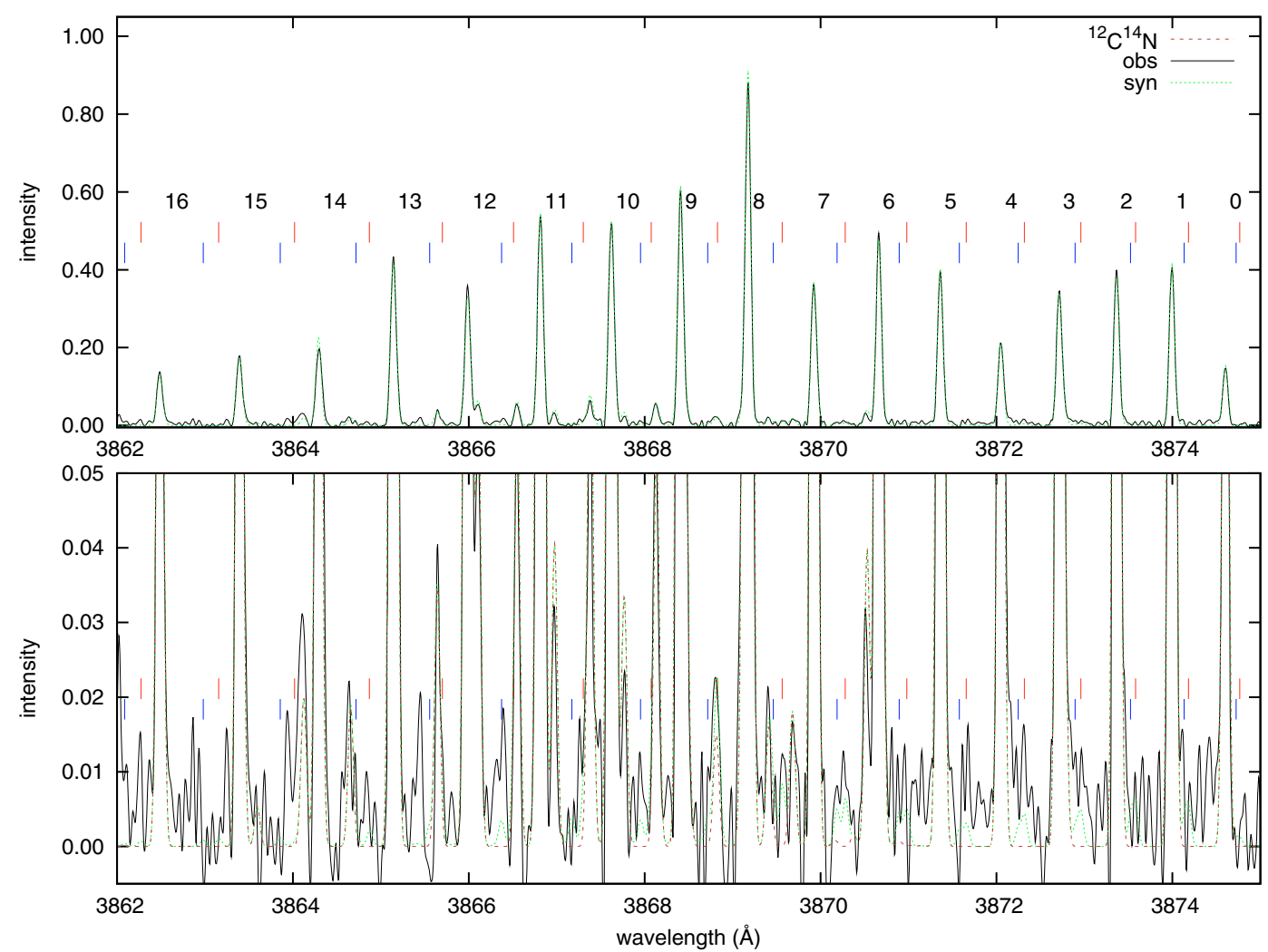

Fig. C.8. Observed (2DCoudé) and synthetic (dotted) spectra of comet C/1999 H1 (Lee).

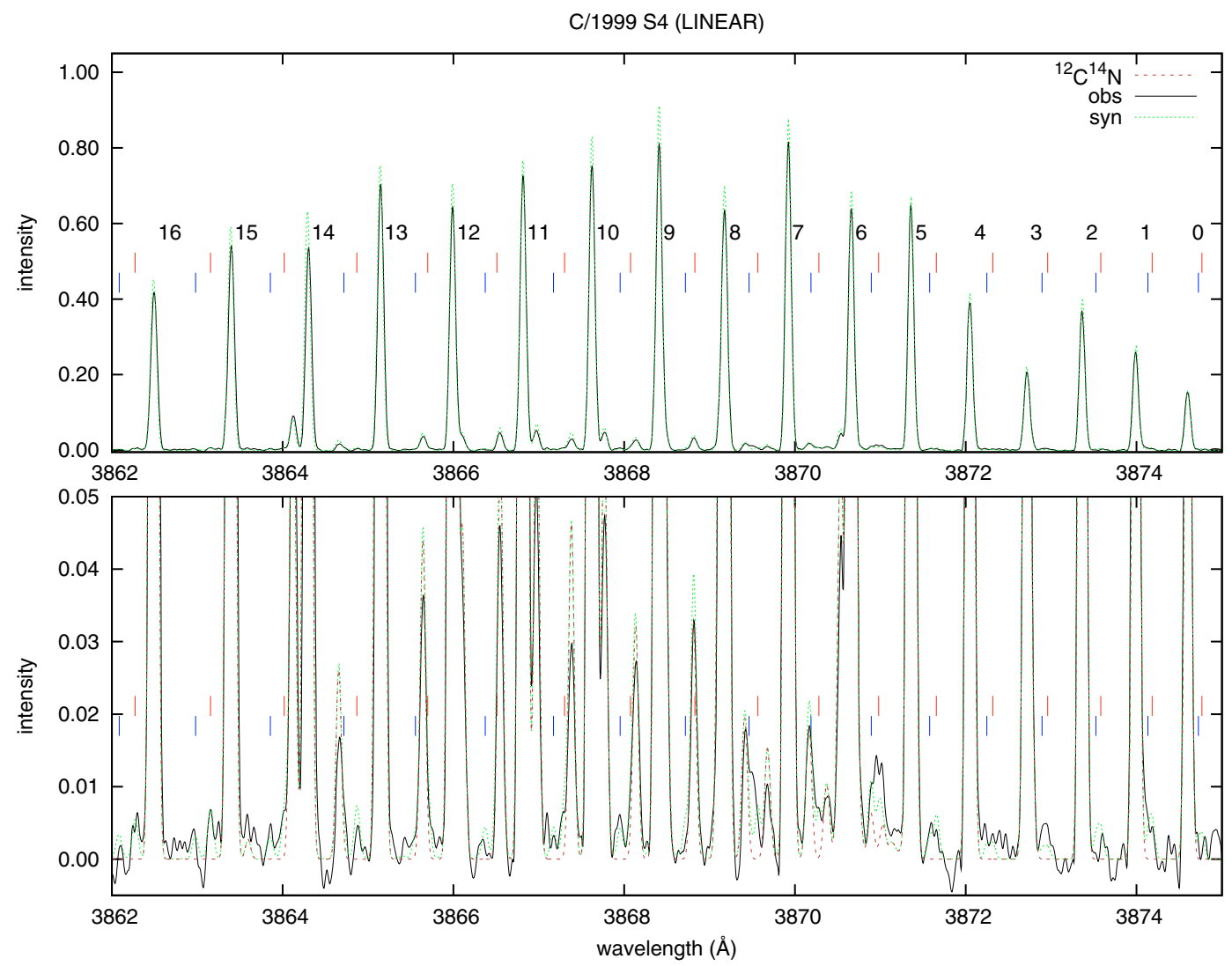

Fig. C.9. Observed (2DCoudé) and synthetic (dotted) spectra of comet C/1999 S4 (LINEAR). 
J. Manfroid et al.: The $\mathrm{CN}$ isotopic ratios in comets, Online Material $p 11$

C/1999 T1 (McNaught-Hartley)
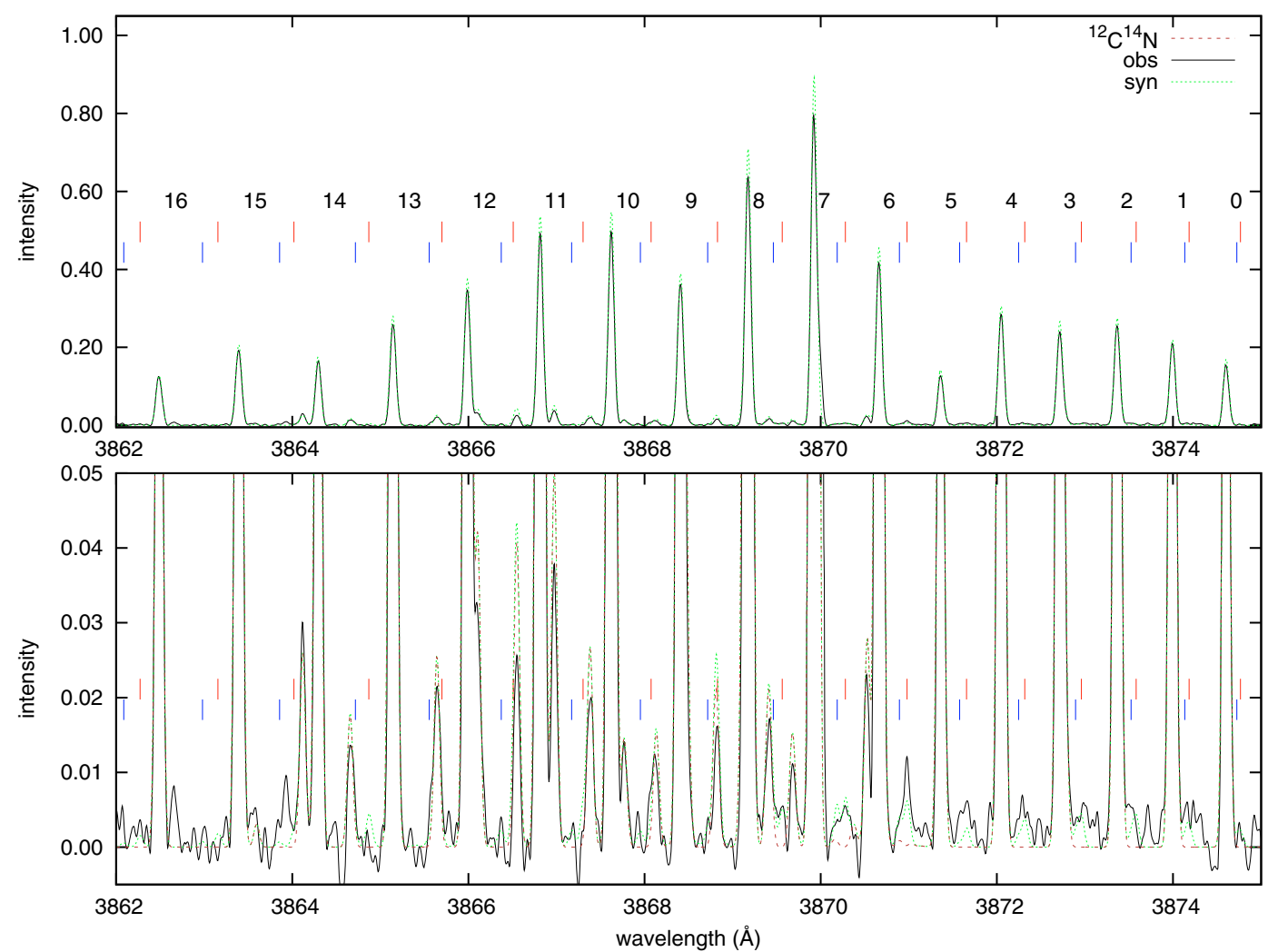

Fig. C.10. Observed (2DCoudé) and synthetic (dotted) spectra of comet C/1999 T1 (McNaught-Hartley).

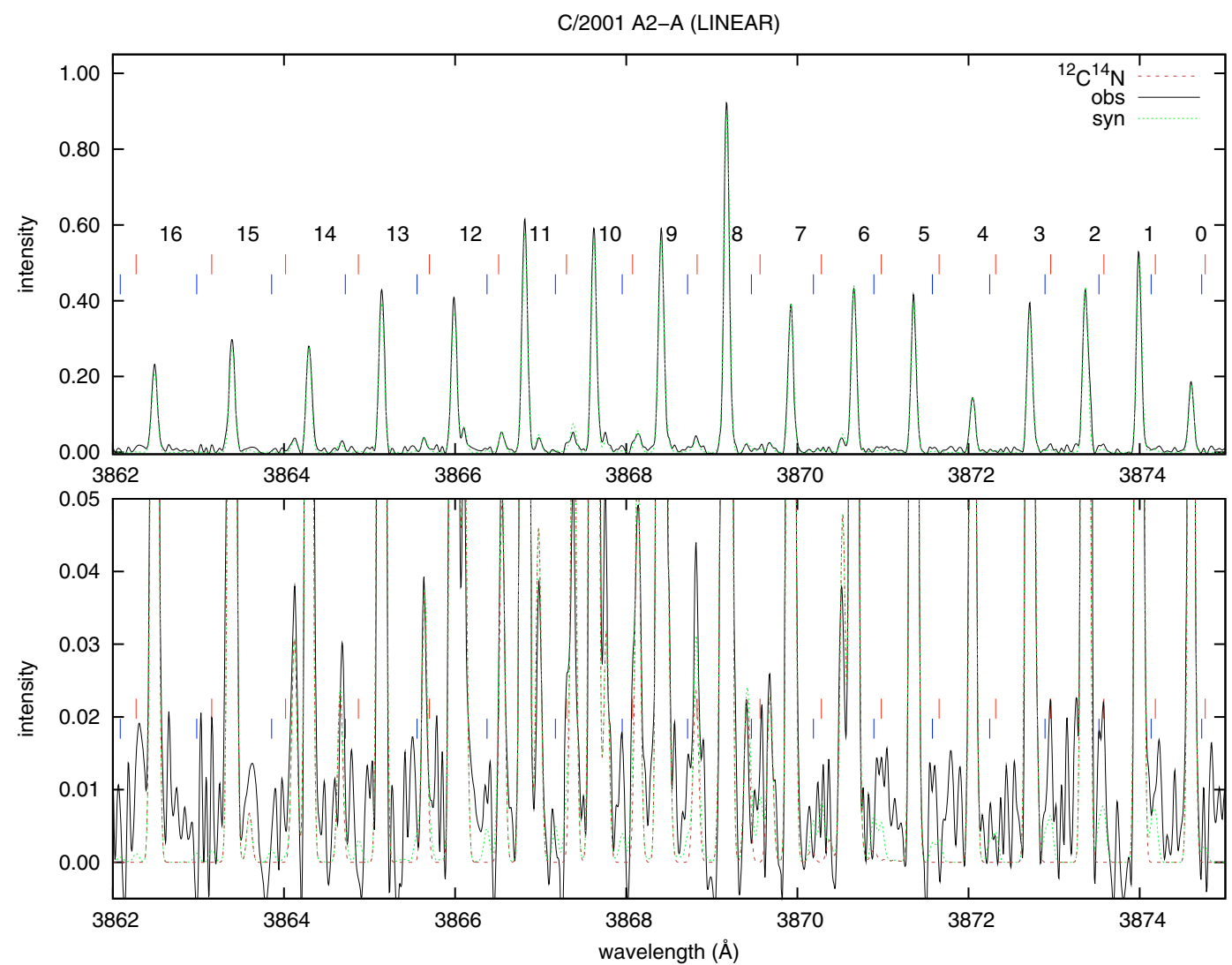

Fig. C.11. Observed (2DCoudé) and synthetic (dotted) spectra of comet C/2001 A2-A (LINEAR). 
J. Manfroid et al.: The $\mathrm{CN}$ isotopic ratios in comets, Online Material p 12

C/2000 WM1 (LINEAR)
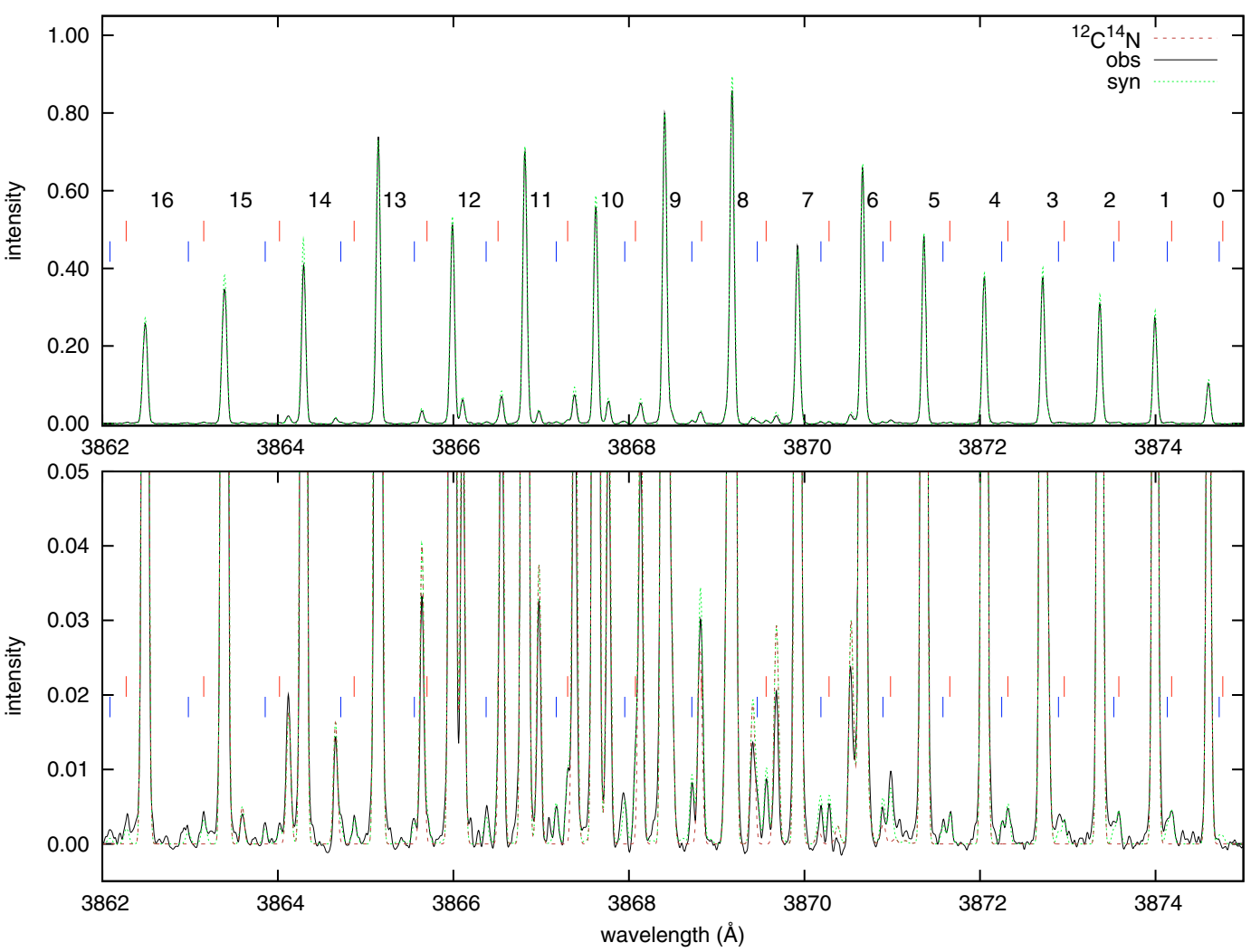

Fig. C.12. Observed (UVES) and synthetic (dotted) spectra of comet C/2000 WM1 (LINEAR).

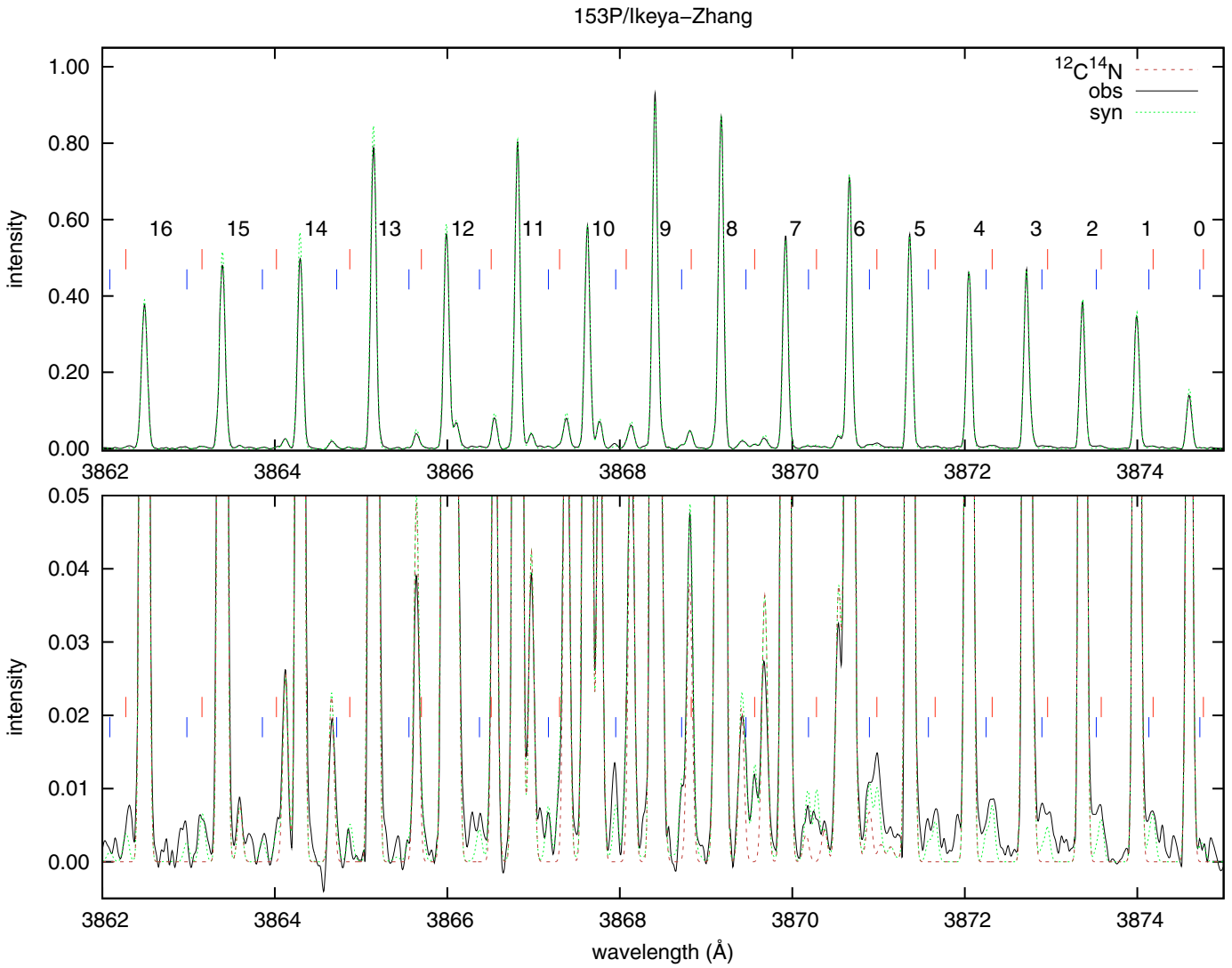

Fig. C.13. Observed (2DCoudé) and synthetic (dotted) spectra of comet 153P/Ikeya-Zhang. 
J. Manfroid et al.: The $\mathrm{CN}$ isotopic ratios in comets, Online Material p 13

C/2002 X5 (Kudo-Fujikawa)
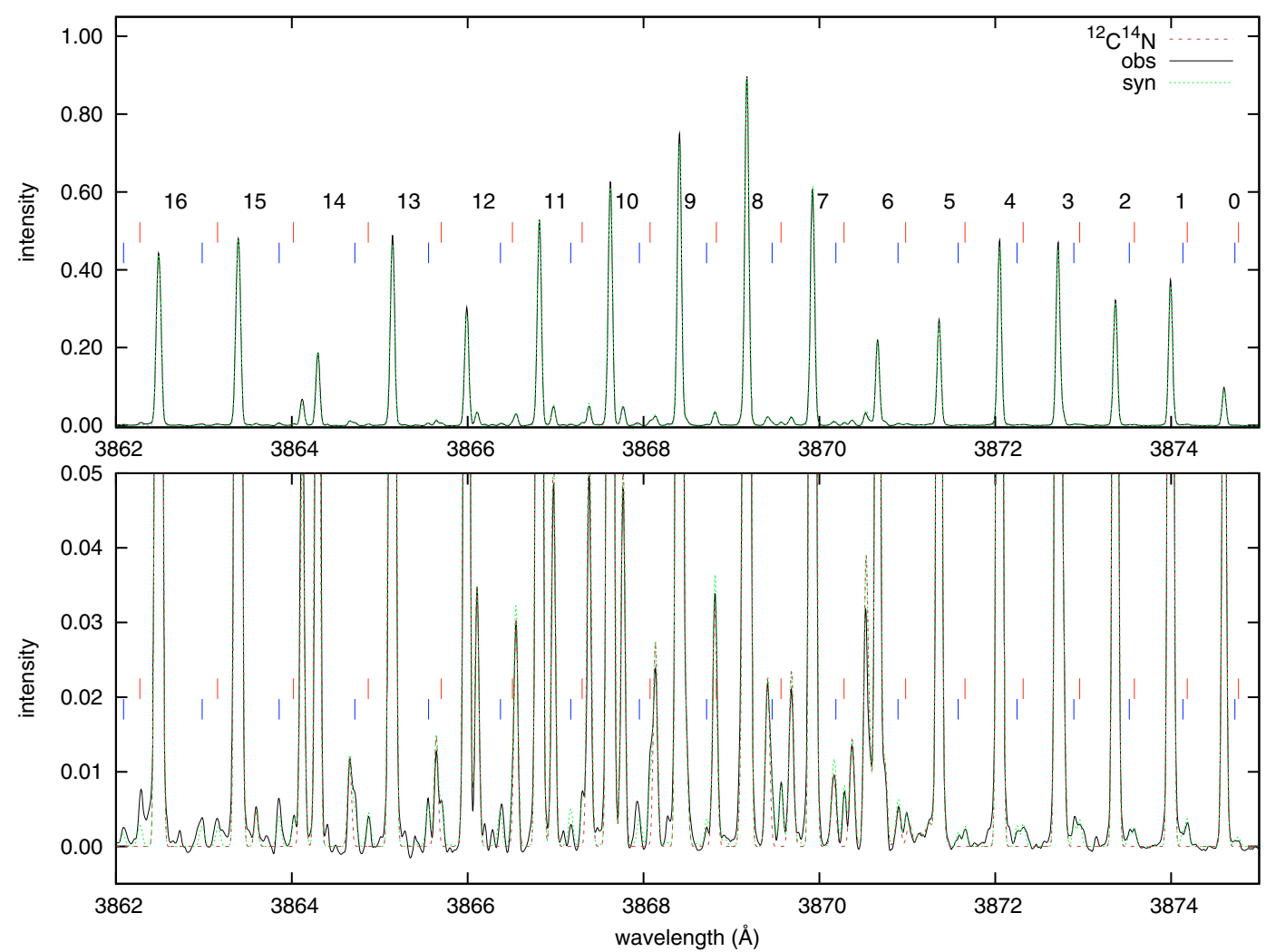

Fig. C.14. Observed (UVES) and synthetic (dotted) spectra of comet C/2002 X5 (Kudo-Fujikawa).

C/2002 V1 (NEAT)
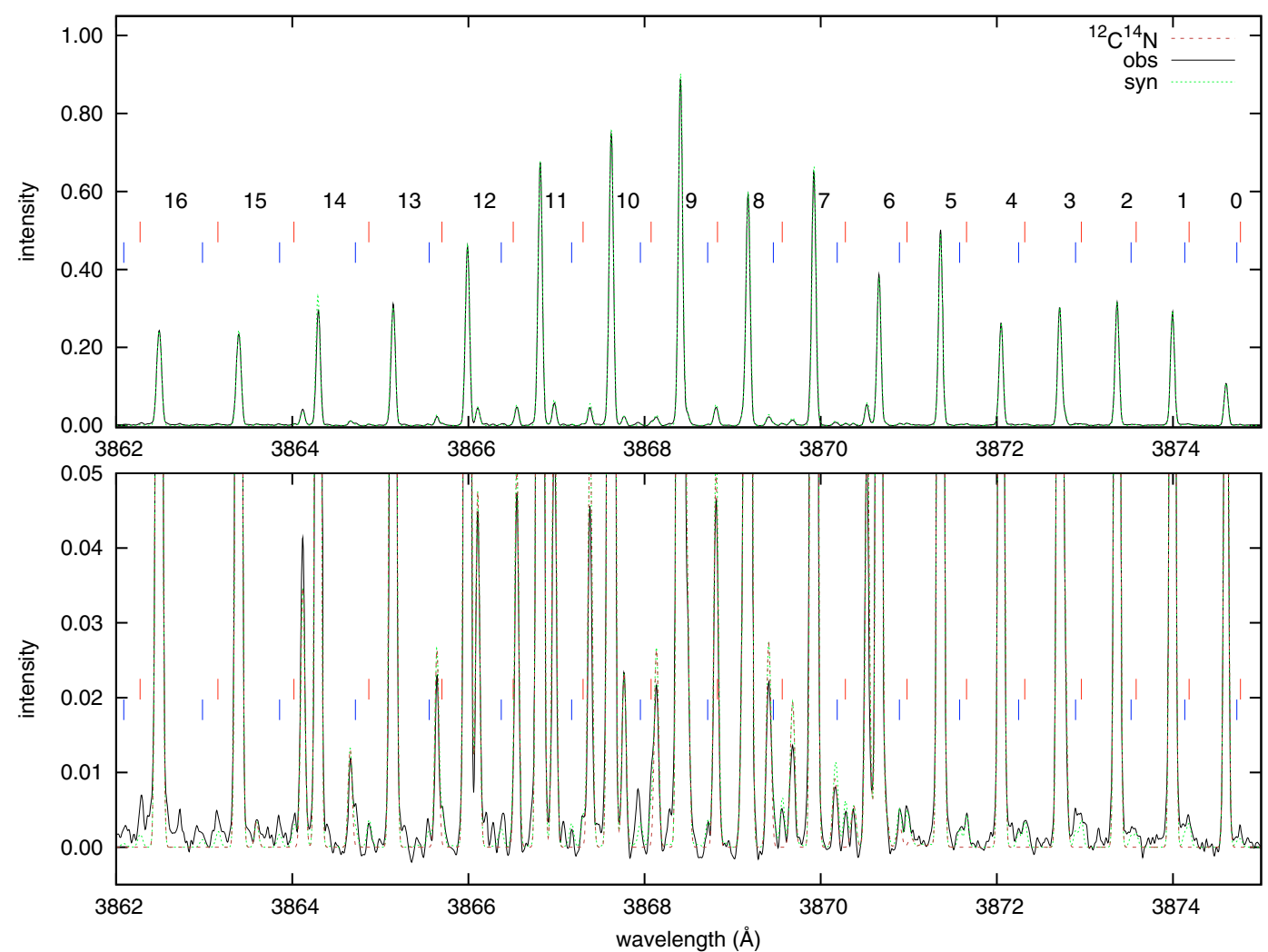

Fig. C.15. Observed (UVES) and synthetic (dotted) spectra of comet C/2002 V1 (NEAT). 
J. Manfroid et al.: The $\mathrm{CN}$ isotopic ratios in comets, Online Material p 14

C/2002 Y1 (Juels-Holvorcem)
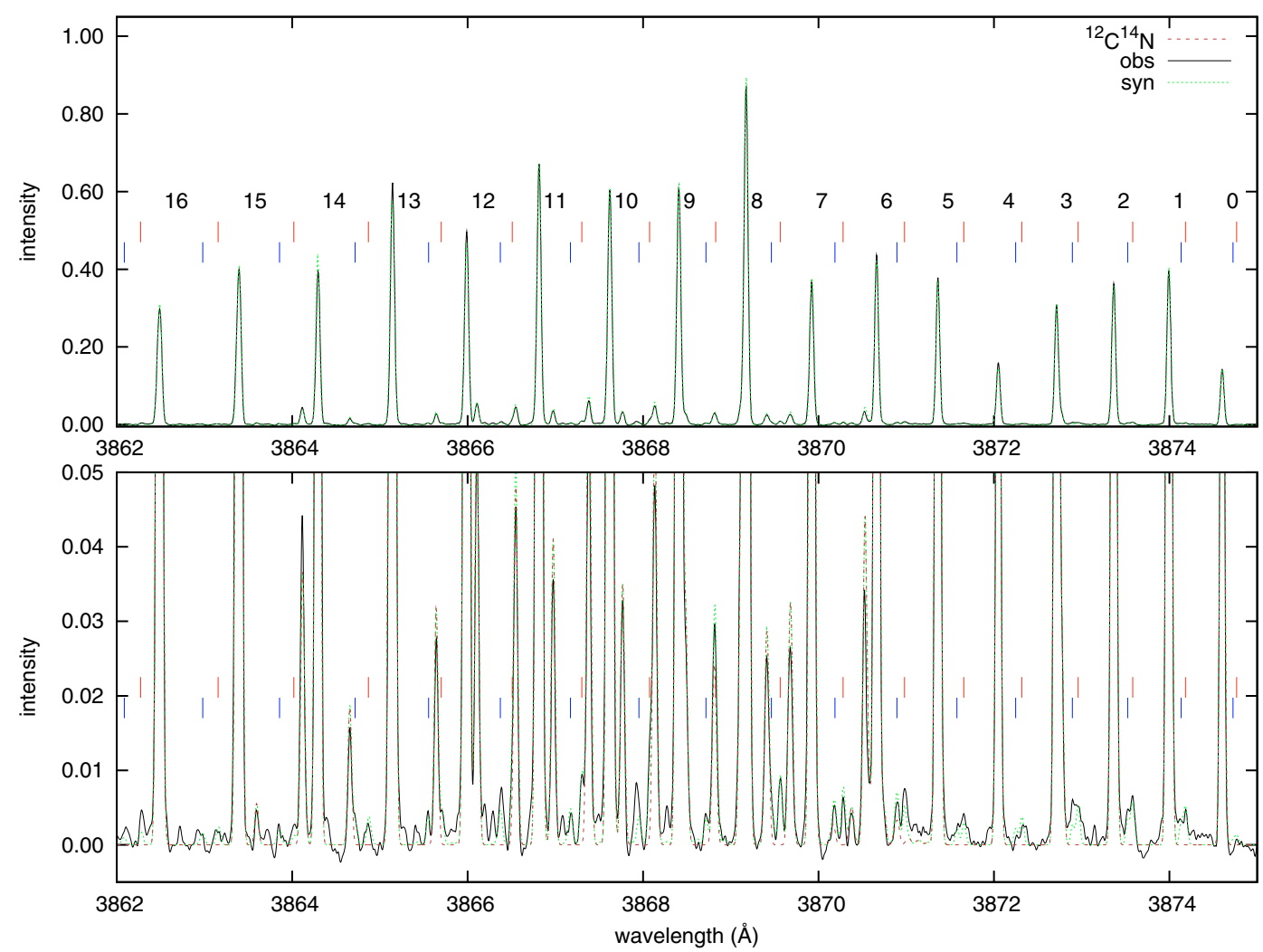

Fig. C.16. Observed (UVES) and synthetic (dotted) spectra of comet C/2002 Y1 (Juels-Holvorcem).

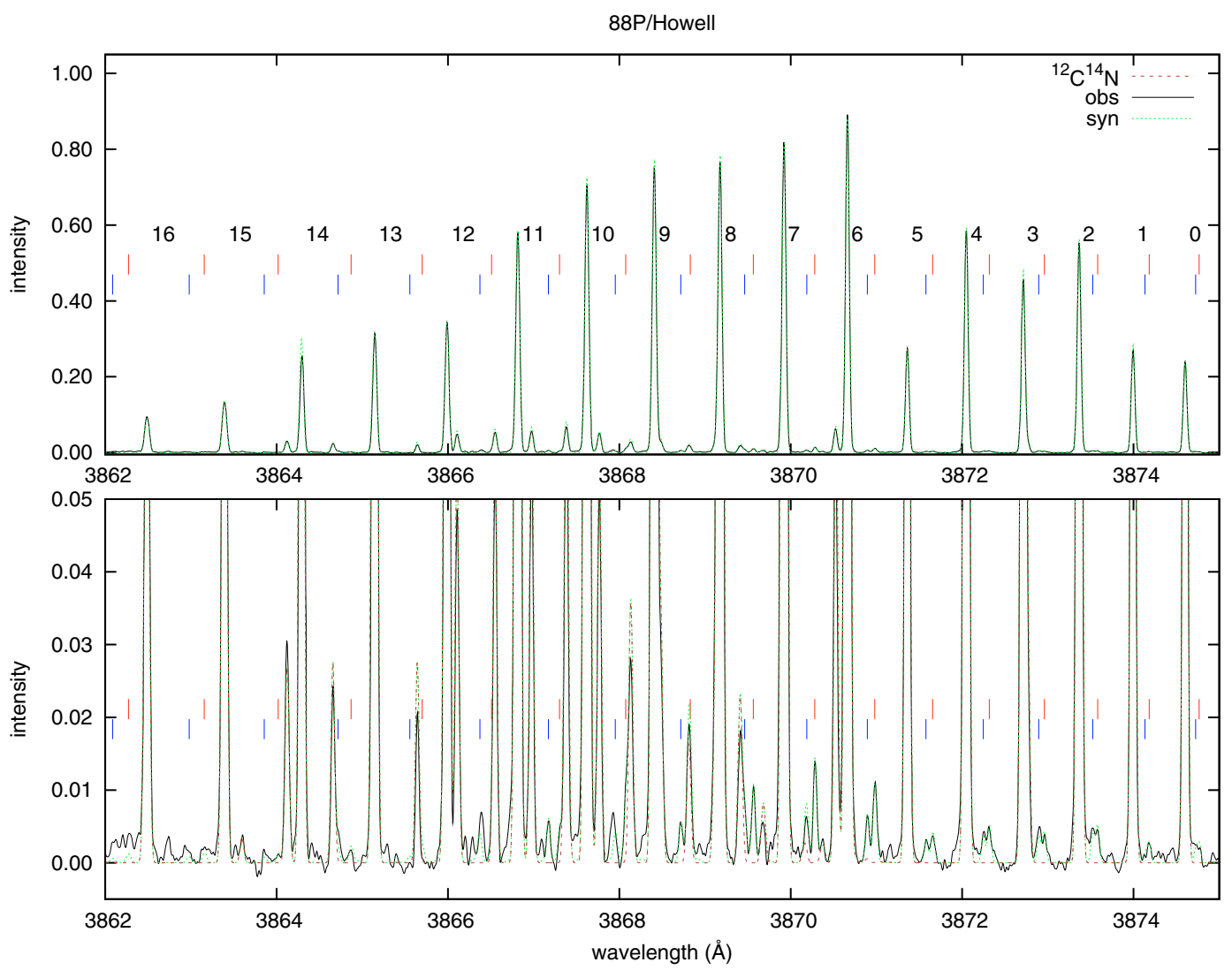

Fig. C.17. Observed (UVES) and synthetic (dotted) spectra of comet 88P/Howell. 
J. Manfroid et al.: The $\mathrm{CN}$ isotopic ratios in comets, Online Material p 15

C/2002 T7 (LINEAR)
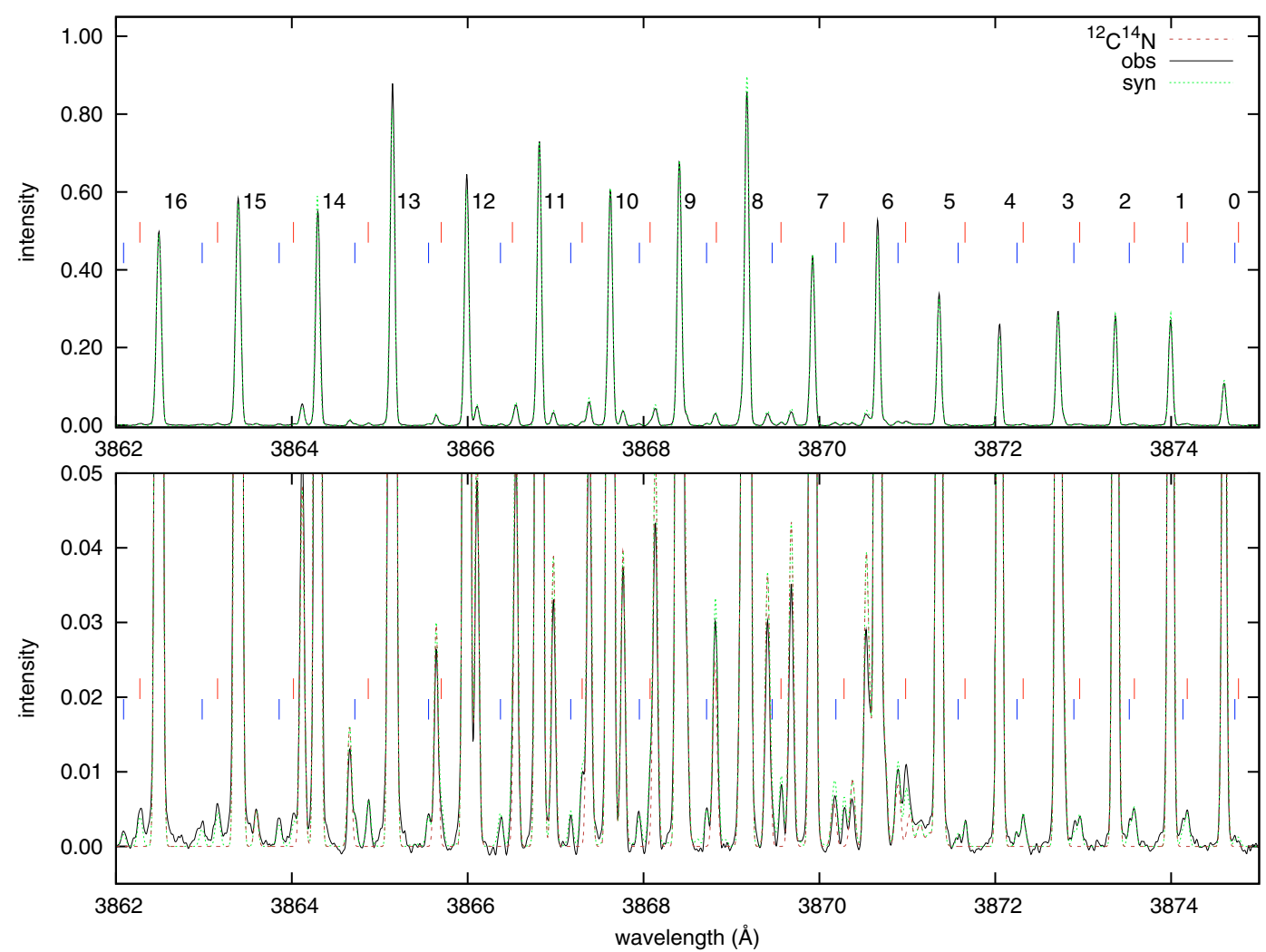

Fig. C.18. Observed (UVES) and synthetic (dotted) spectra of comet C/2002 T7 (LINEAR).

C/2001 Q4 (NEAT)
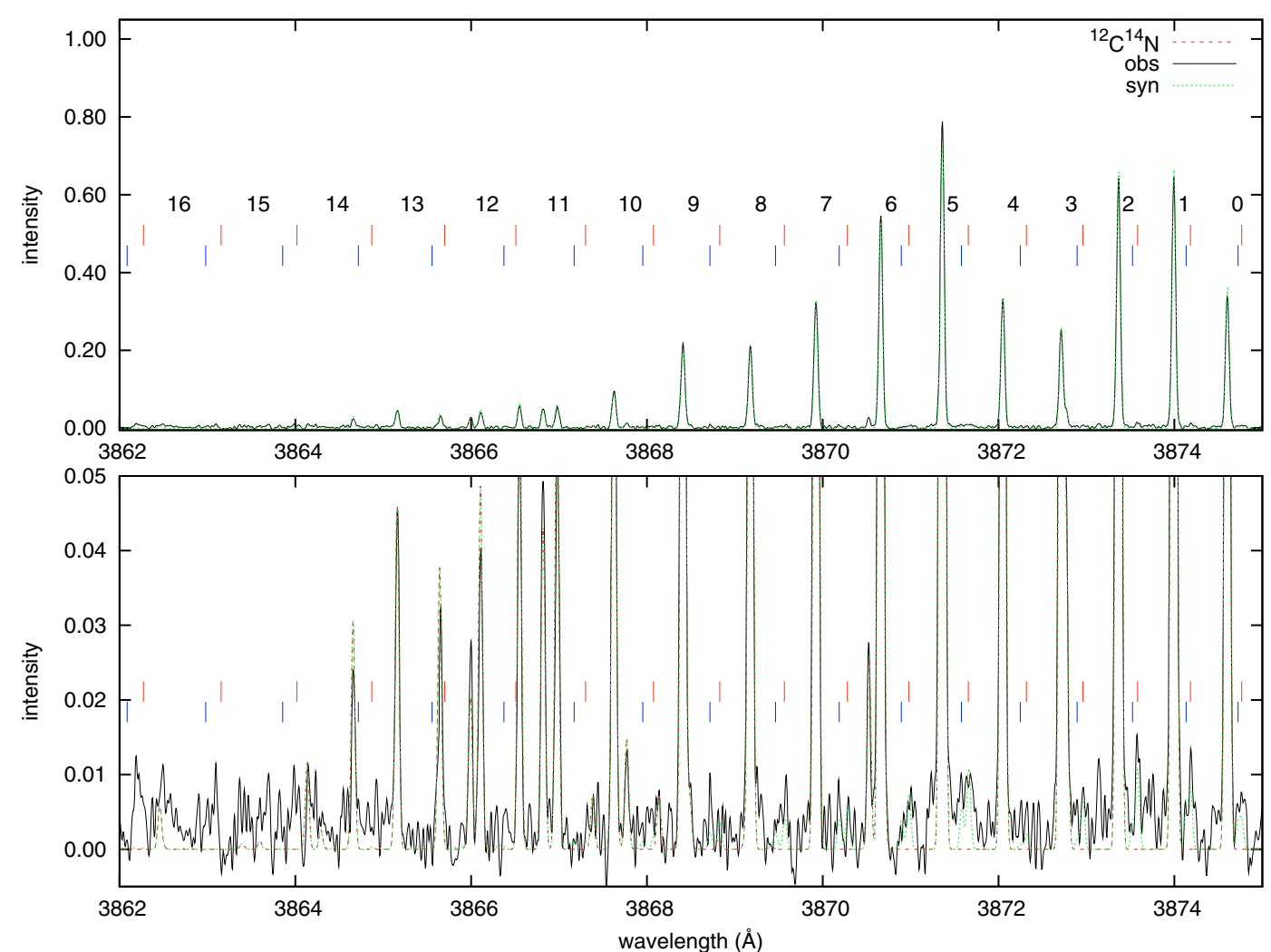

Fig. C.19. Observed (UVES) and synthetic (dotted) spectra of comet C/2001 Q4 (NEAT). 
J. Manfroid et al.: The $\mathrm{CN}$ isotopic ratios in comets, Online Material p 16

C/2001 Q4 (NEAT)
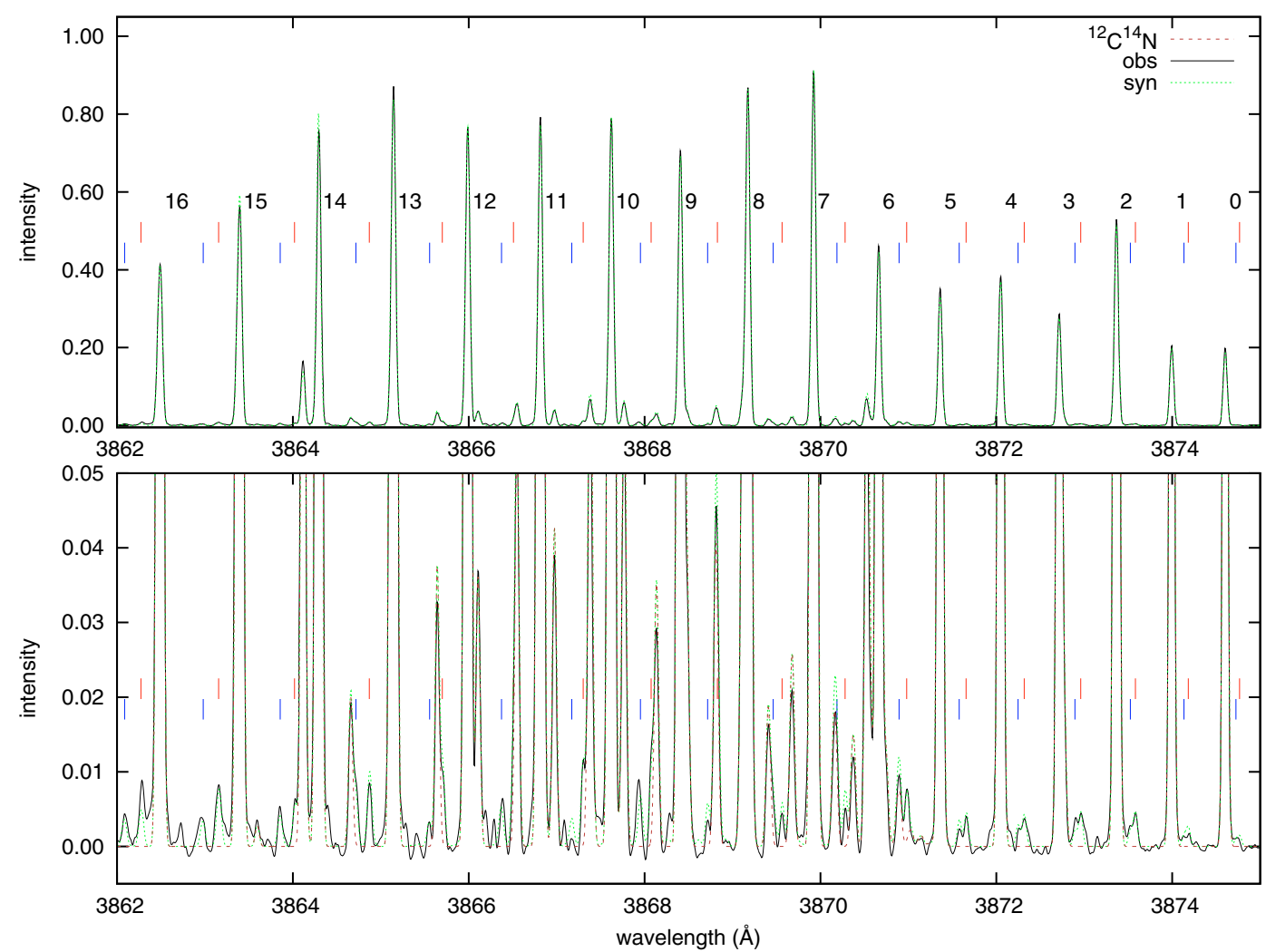

Fig. C.20. Observed (UVES) and synthetic (dotted) spectra of comet C/2001 Q4 (NEAT).

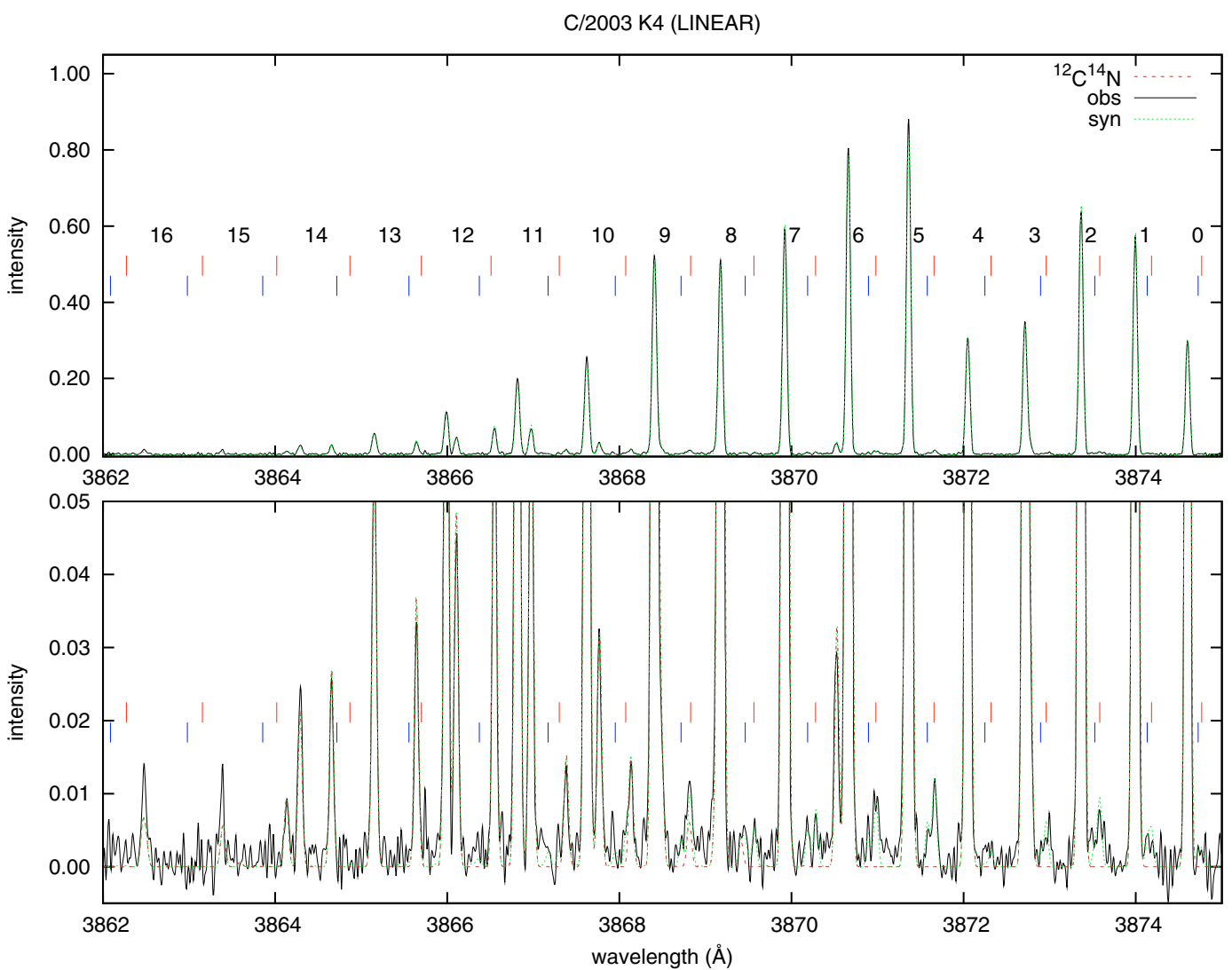

Fig. C.21. Observed (UVES) and synthetic (dotted) spectra of comet C/2003 K4 (LINEAR). 
J. Manfroid et al.: The $\mathrm{CN}$ isotopic ratios in comets, Online Material p 17

C/2003 K4 (LINEAR)
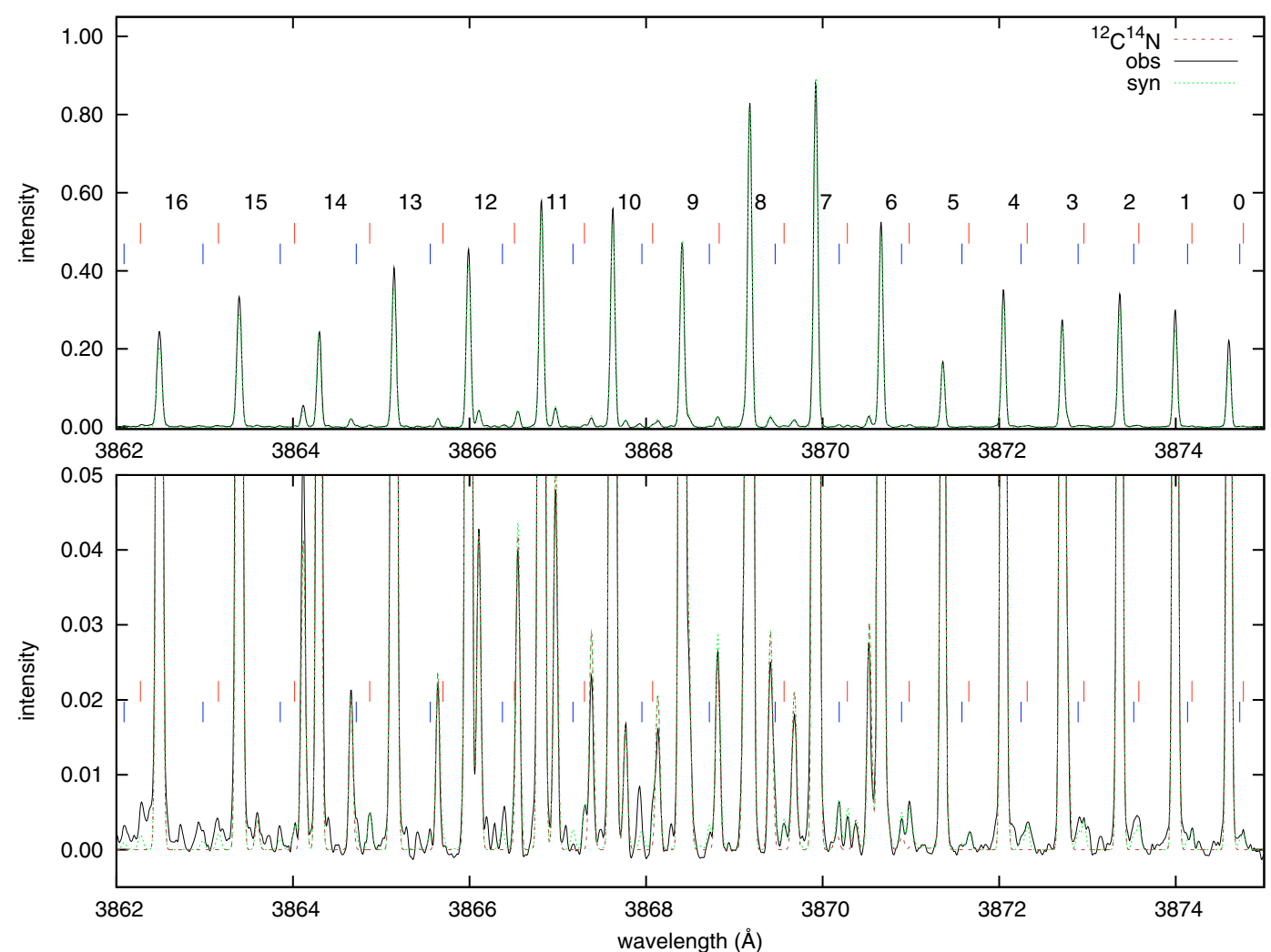

Fig. C.22. Observed (UVES) and synthetic (dotted) spectra of comet C/2003 K4 (LINEAR).

9P/Tempel 1
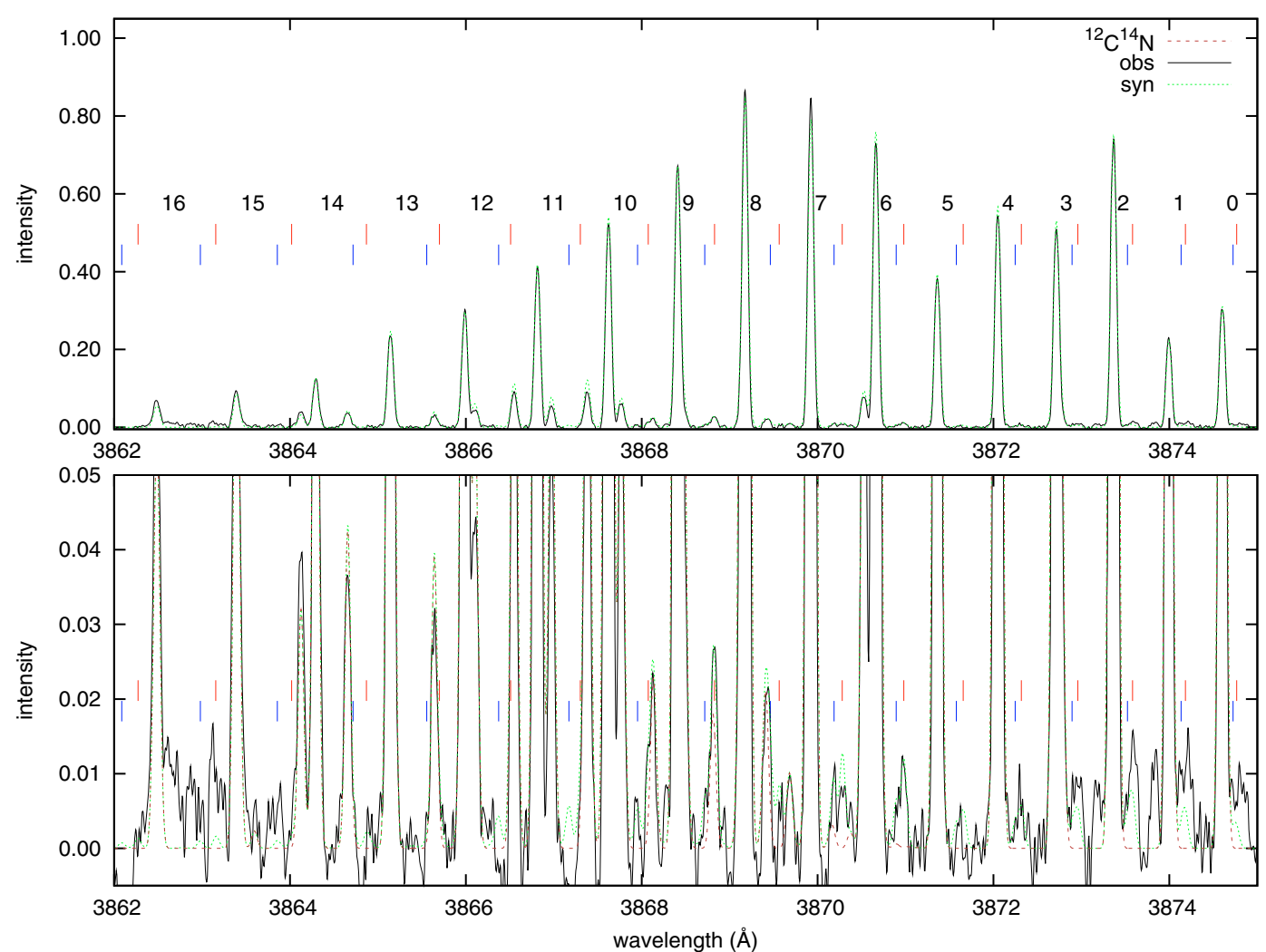

Fig. C.23. Observed (HIRES) and synthetic (dotted) spectra of comet 9P/Tempel 1. 
J. Manfroid et al.: The $\mathrm{CN}$ isotopic ratios in comets, Online Material p 18

9P/Tempel 1
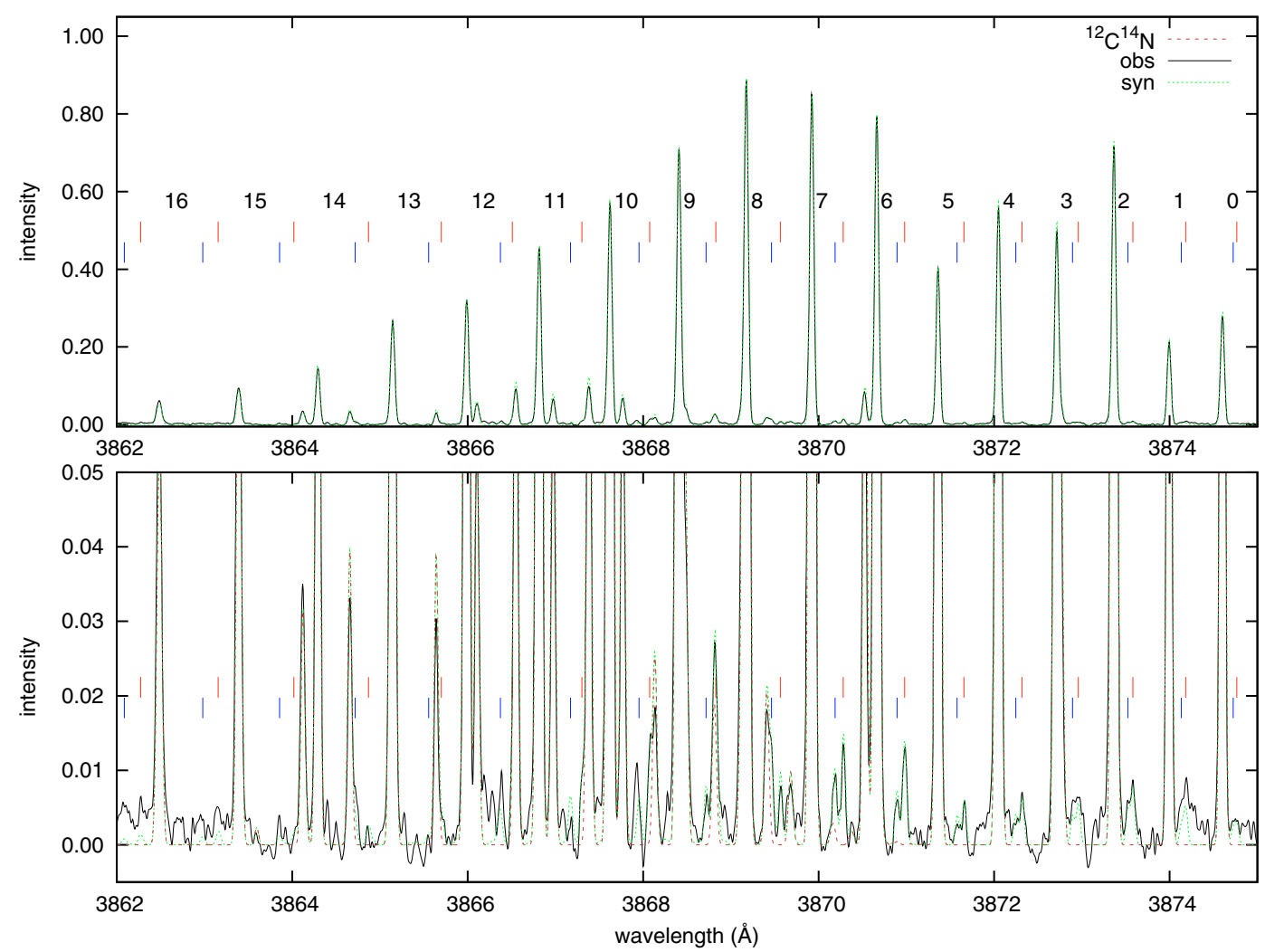

Fig. C.24. Observed (UVES) and synthetic (dotted) spectra of comet 9P/Tempel 1.

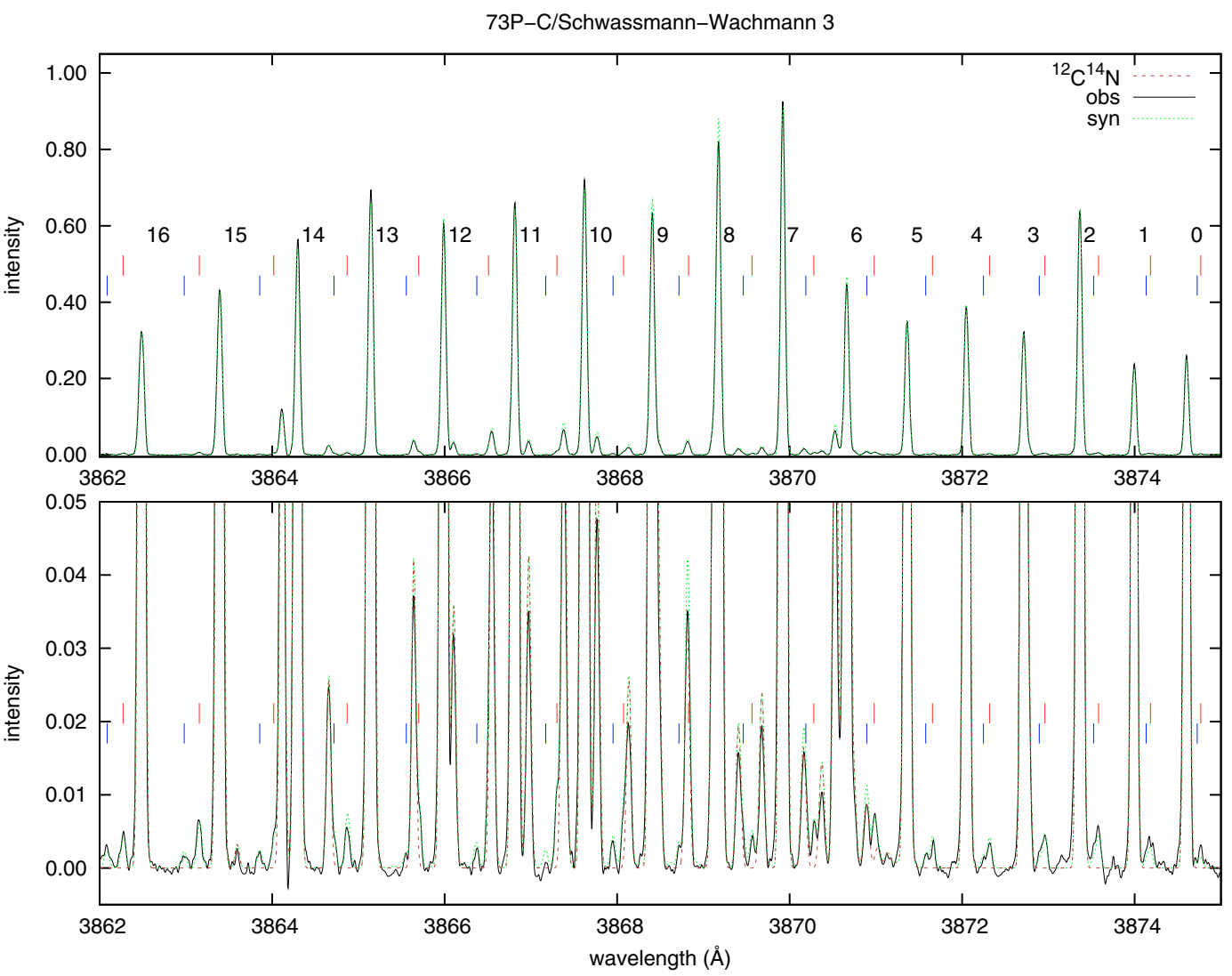

Fig. C.25. Observed (UVES) and synthetic (dotted) spectra of comet 73P-B/Schwassmann-Wachmann 3. 
J. Manfroid et al.: The CN isotopic ratios in comets, Online Material p 19

73P-C/Schwassmann-Wachmann 3
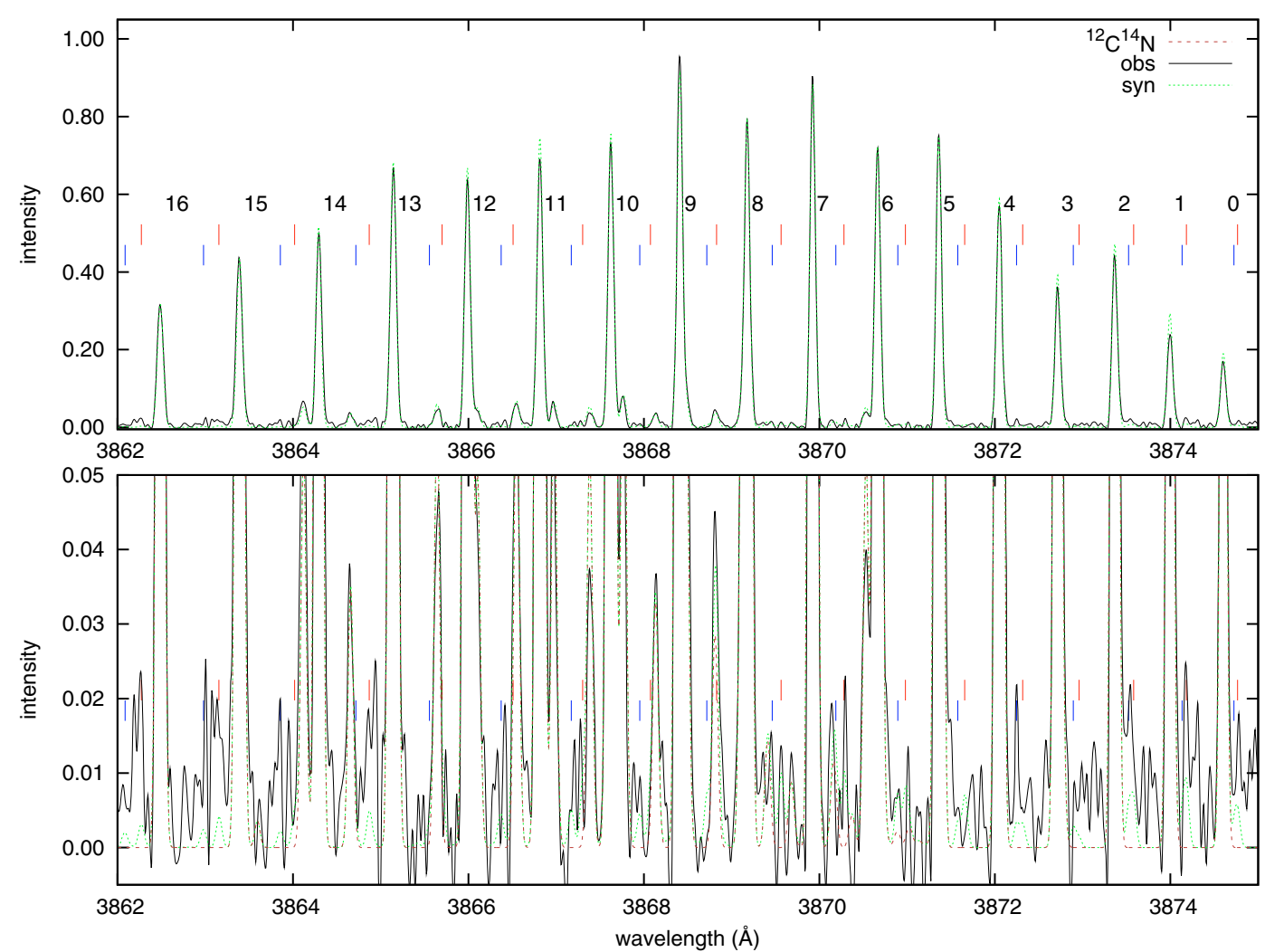

Fig. C.26. Observed (2DCoudé) and synthetic (dotted) spectra of comet 73P-B/Schwassmann-Wachmann 3.
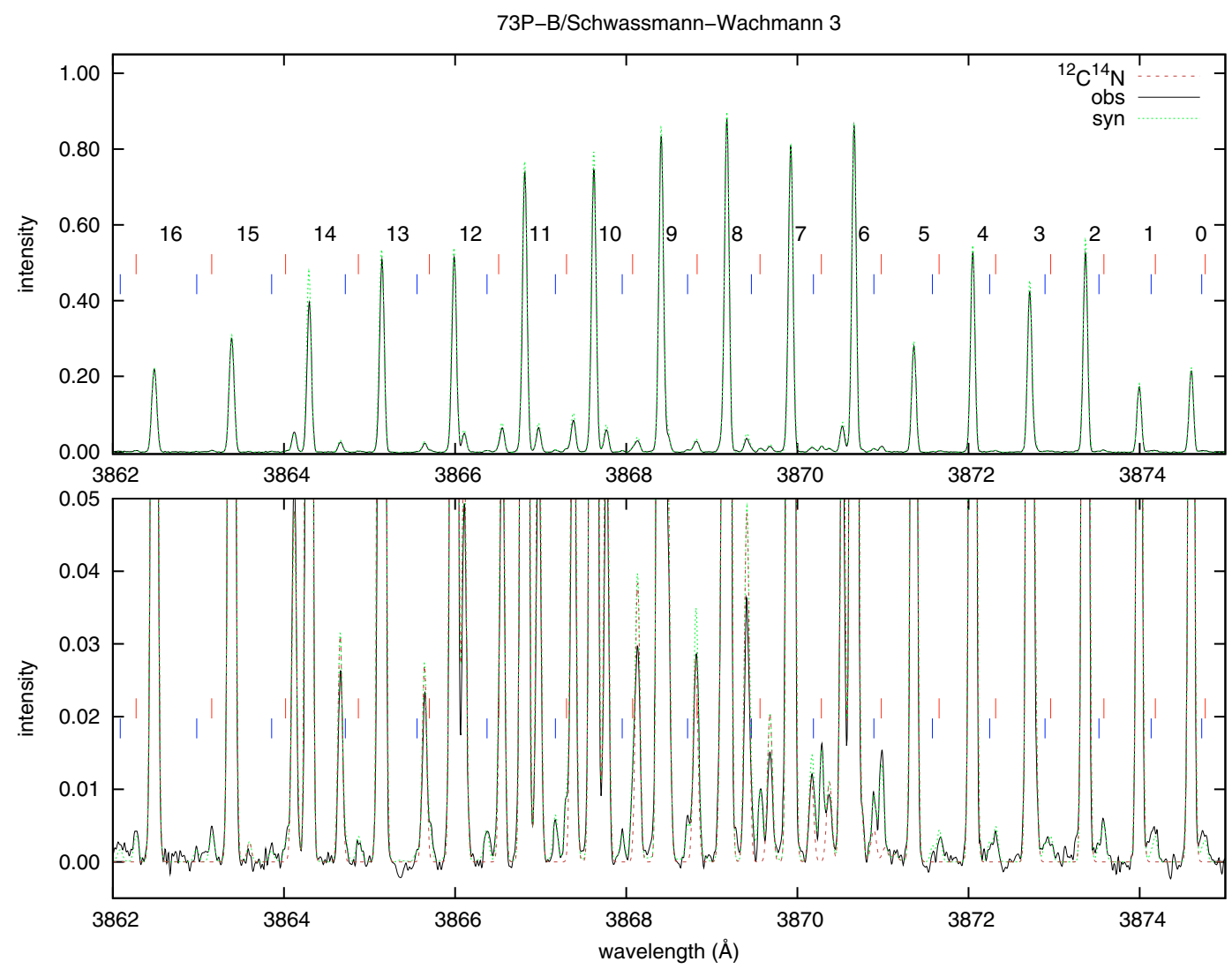

Fig. C.27. Observed (UVES) and synthetic (dotted) spectra of comet 73P-C/Schwassmann-Wachmann 3. 
J. Manfroid et al.: The $\mathrm{CN}$ isotopic ratios in comets, Online Material p 20

73P-B/Schwassmann-Wachmann 3
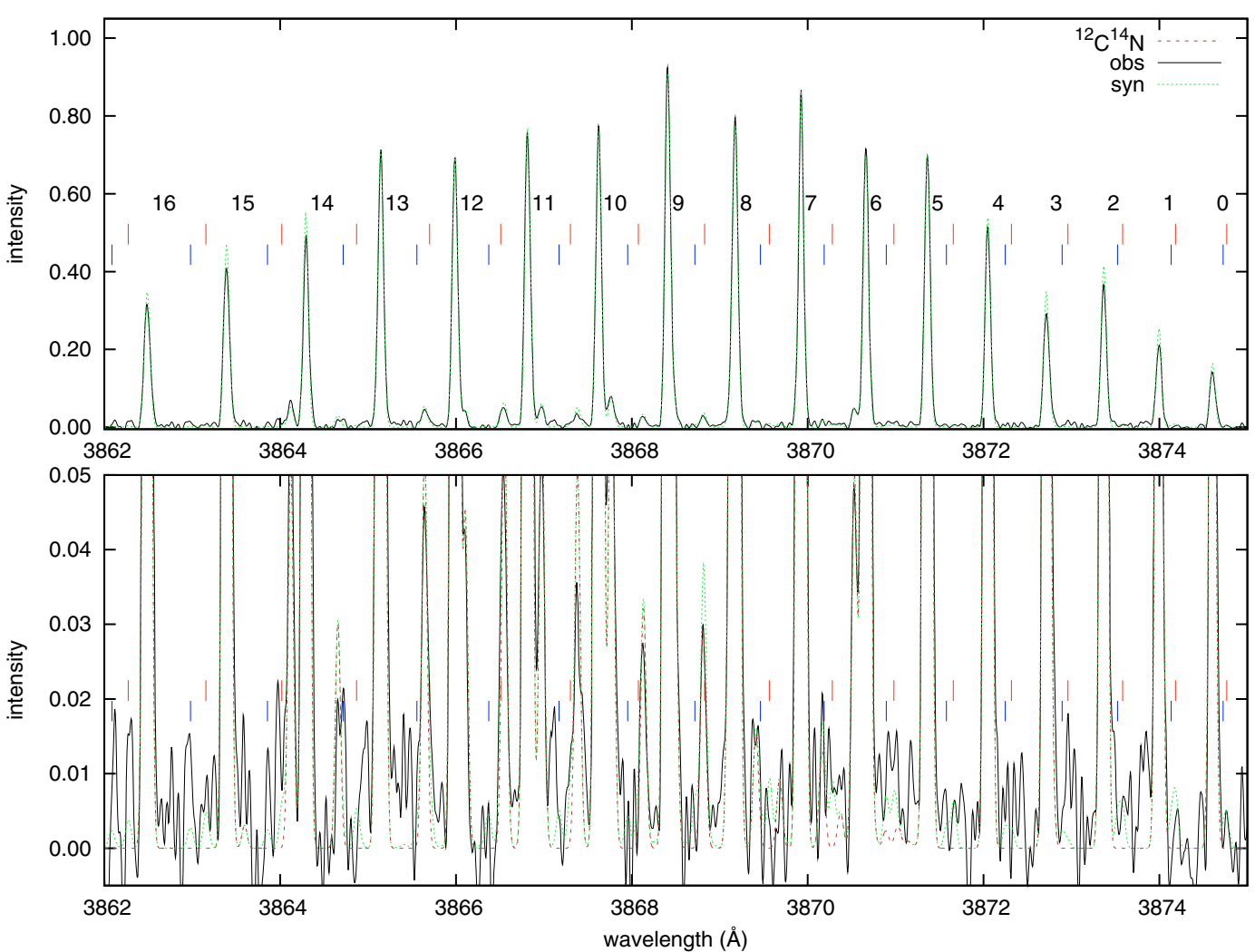

Fig. C.28. Observed (2DCoudé) and synthetic (dotted) spectra of comet 73P-C/Schwassmann-Wachmann 3.

C/2006 M4 (SWAN)
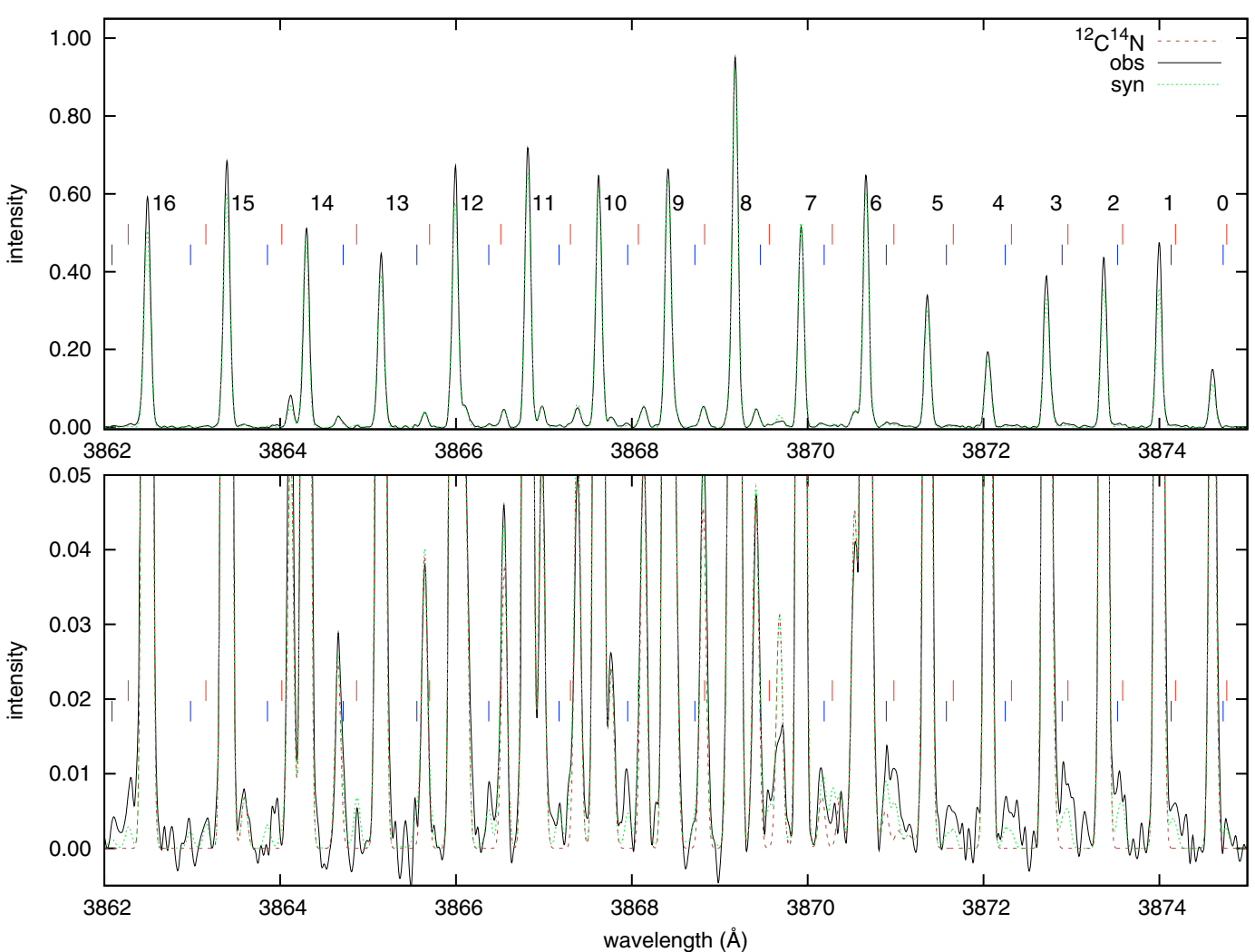

Fig. C.29. Observed (2DCoudé) and synthetic (dotted) spectra of comet C/2006 M4 (SWAN). 
J. Manfroid et al.: The CN isotopic ratios in comets, Online Material p 21
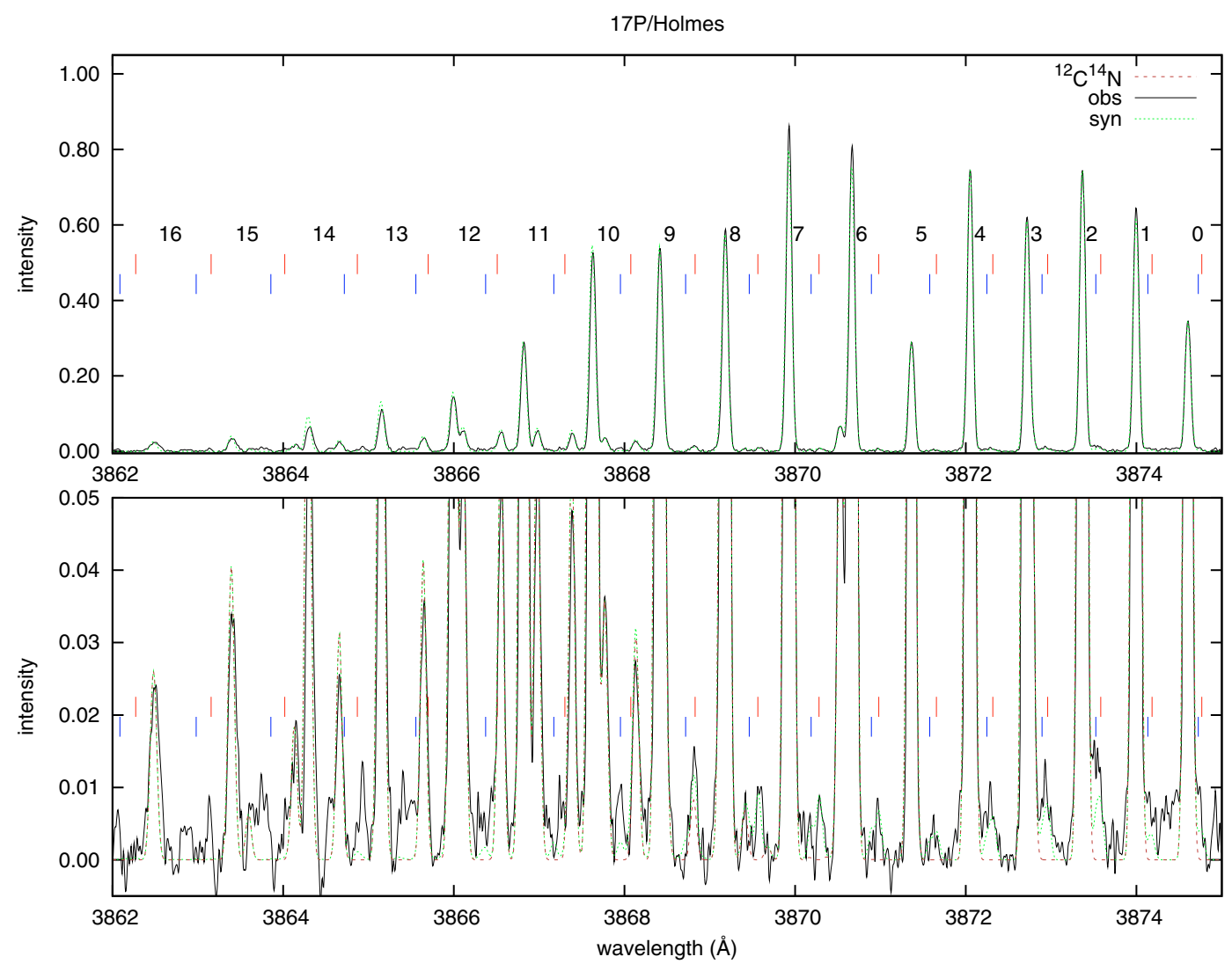

Fig. C.30. Observed (HIRES) and synthetic (dotted) spectra of comet 17P/Holmes.

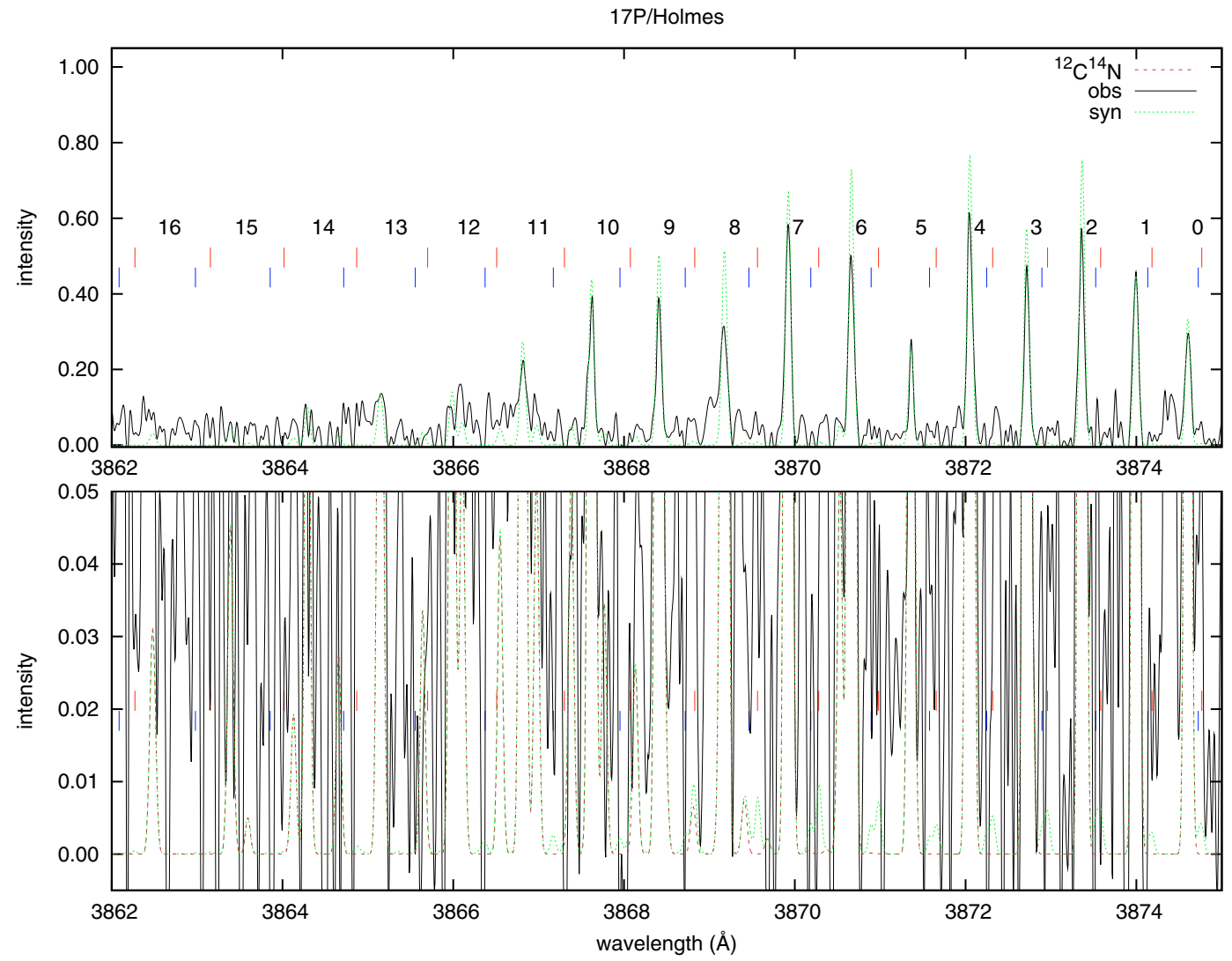

Fig. C.31. Observed (2DCoudé) and synthetic (dotted) spectra of comet 17P/Holmes. 
J. Manfroid et al.: The $\mathrm{CN}$ isotopic ratios in comets, Online Material p 22

8P/Tuttle
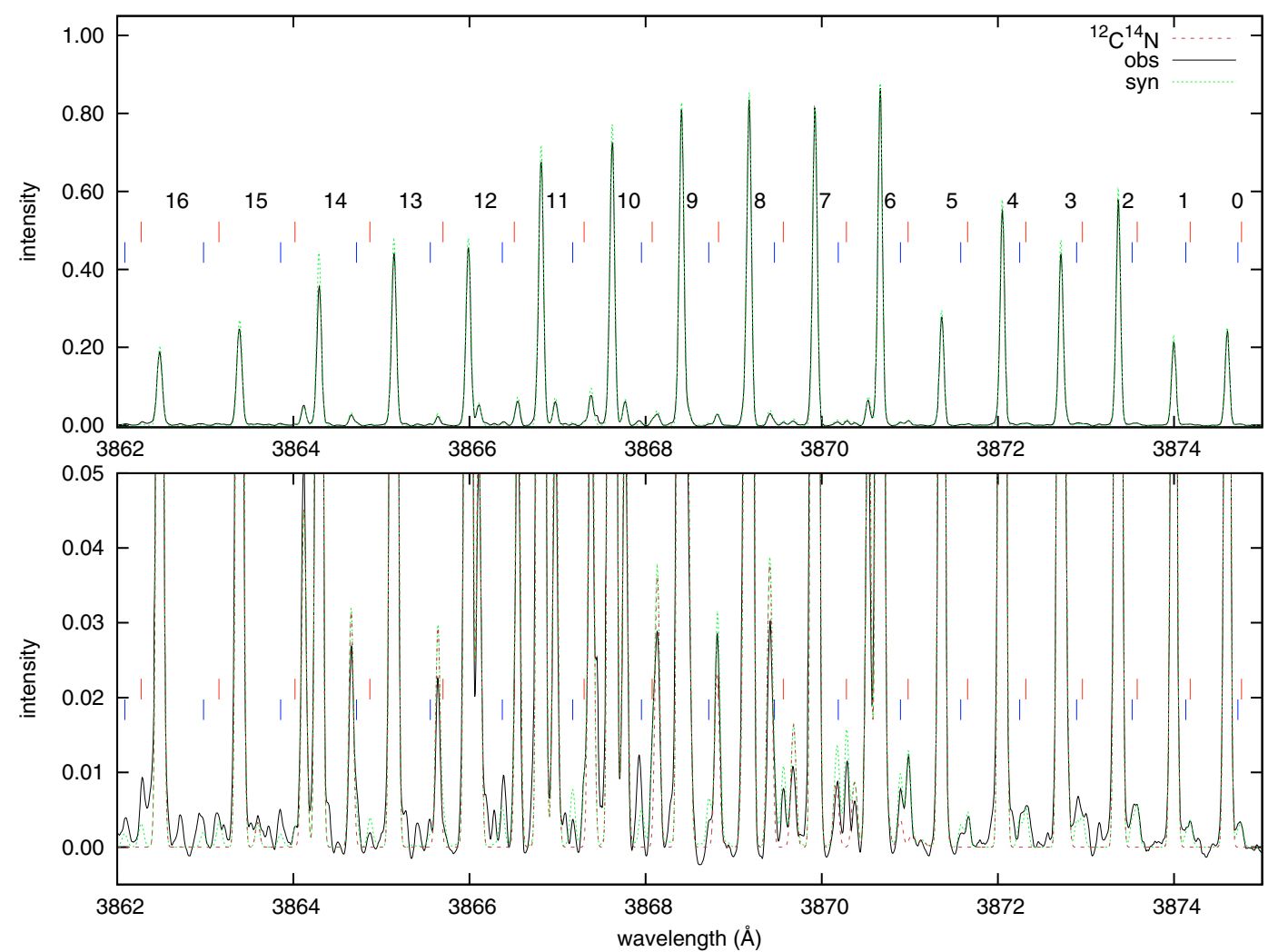

Fig. C.32. Observed (2DCoudé) and synthetic (dotted) spectra of comet 8P/Tuttle.

C/2007 N3 (Lulin)
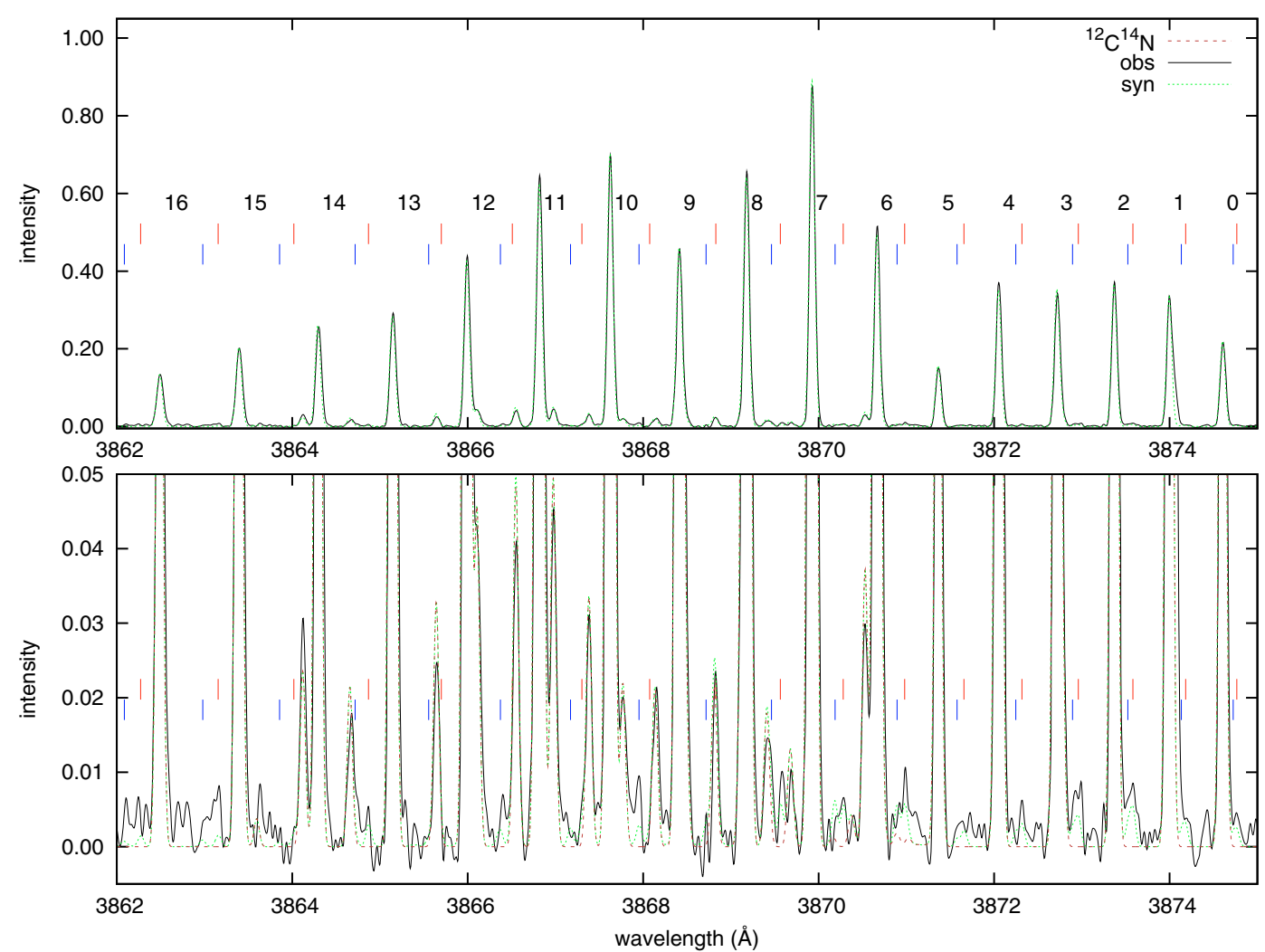

Fig. C.33. Observed (2DCoudé) and synthetic (dotted) spectra of comet C/2007 N3 (Lulin). 
J. Manfroid et al.: The $\mathrm{CN}$ isotopic ratios in comets, Online Material p 23

\section{Appendix D: Synthetic spectra}

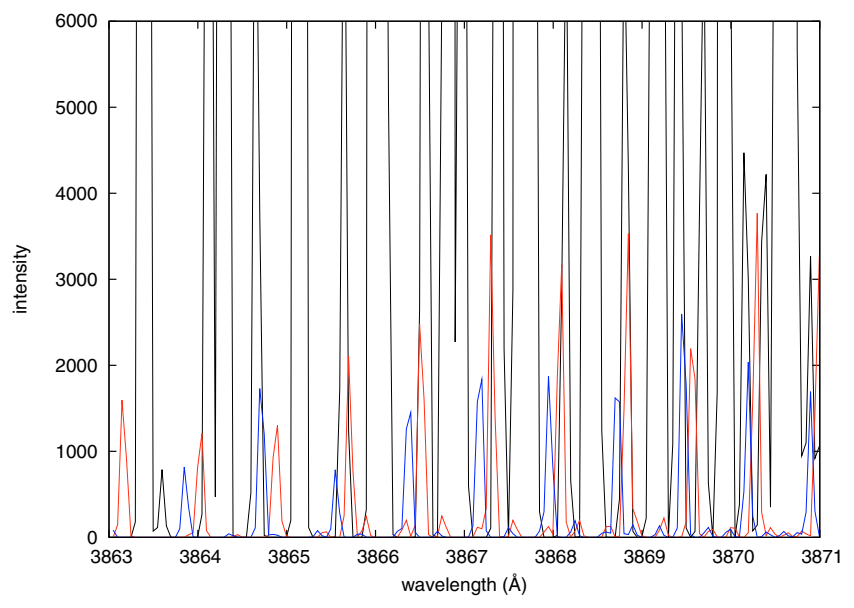

Fig. D.1. Small region of the B-X $0-0$ band of the three $\mathrm{CN}$ isotopologues. The synthetic spectra were computed using isotopic abundances of $1,1 / 89$ and $1 / 145$ for ${ }^{12} \mathrm{C}^{14} \mathrm{~N}$ (black), ${ }^{13} \mathrm{C}^{14} \mathrm{~N}$ (red) and ${ }^{12} \mathrm{C}^{15} \mathrm{~N}$ (blue), respectively, in the absence of collisional effects, and with $r=1 \mathrm{AU}$, $\dot{r}=0$. The Gaussian profile has $F W H M=0.05 \AA$. The intensity scale is arbitrary, but coherent with the three following plots.

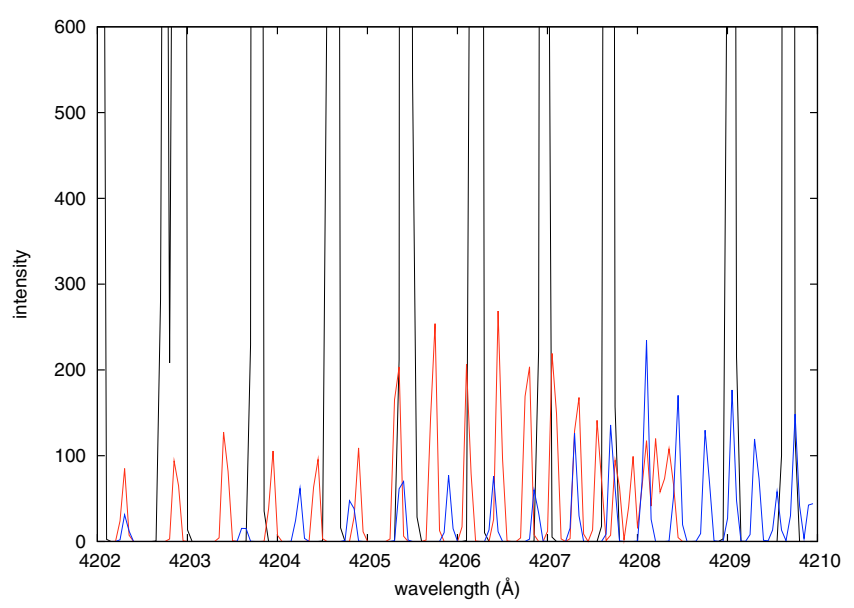

Fig. D.2. Same as Fig. D.1 for a region of the B-X 0-1 band.

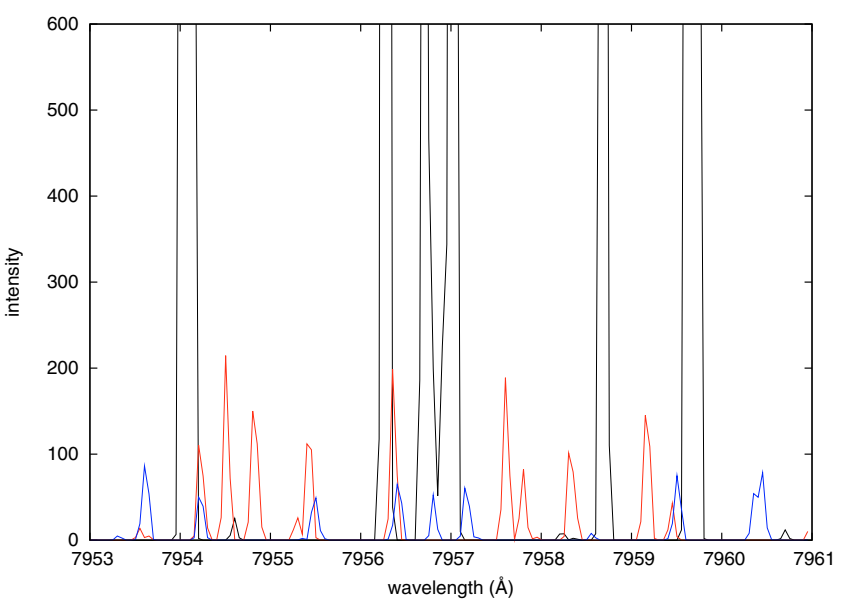

Fig. D.3. Same as Fig. D.1 for a region of the A-X 2-0 band.

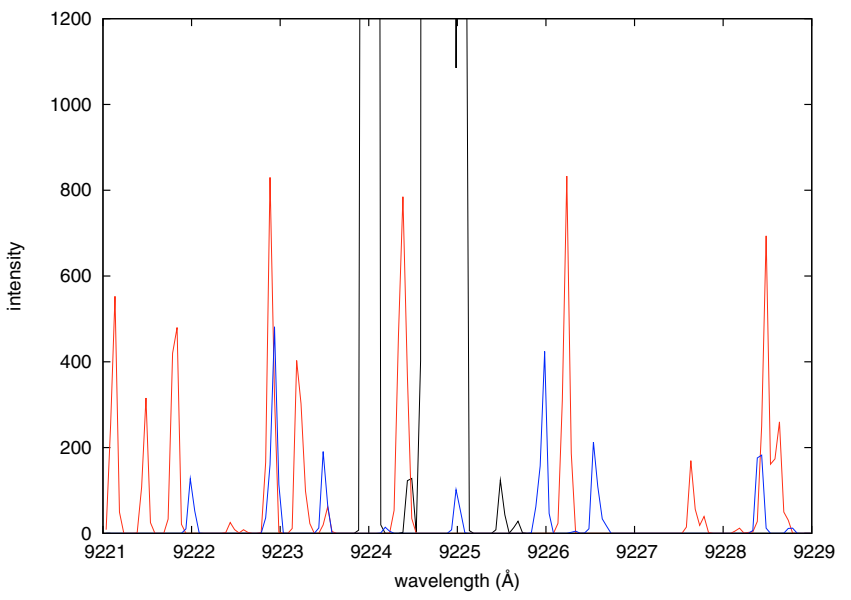

Fig. D.4. Same as Fig. D.1 for a region of the A-X 1-0 band. 